The National Bureau of Standards ' was established by an act of Congress March 3, 1901. The Bureau's overall goal is to strengthen and advance the Nation's science and technology and facilitate their effective application for public benefit. To this end, the Bureau conducts research and provides: (1) a basis for the Nation's physical measurement system, (2) scientific and technological services for industry and government, (3) a technical basis for equity in trade, and (4) technical services to promote public safety. The Bureau consists of the Institute for Basic Standards, the Institute for Materials Research, the Institute for Applied Technology, the Institute for Computer Sciences and Technology, and the Office for Information Programs.

THE INSTITUTE FOR BASIC STANDARDS provides the central basis within the United States of a complete and consistent system of physical measurement; coordinates that system with measurement systems of other nations; and furnishes essential services leading to accurate and uniform physical measurements throughout the Nation's scientific community, industry, and commerce. The Institute consists of a Center for Radiation Research, an Office of Measurement Services and the following divisions:

Applied Mathematics - Electricity - Mechanics - Heat - Optical Physics - Nuclear Sciences" - Applied Radiation " - Quantum Electronics ${ }^{3}$ - Electromagnetics ${ }^{3}$ - Time and Frequency " - Laboratory Astrophysics " - Cryogenics".

THE INSTITUTE FOR MATERIALS RESEARCH conducts materials research leading to improved methods of measurement, standards, and data on the properties of well-characterized materials needed by industry, commerce, educational institutions, and Government; provides advisory and research services to other Government agencies; and develops, produces, and distributes standard reference materials. The Institute consists of the Office of Standard Reference Materials and the following divisions:

Analytical Chemistry - Polymers - Metallurgy - Inorganic Materials - Reactor Radiation - Physical Chemistry.

THE INSTITUTE FOR APPLIED TECHNOLOGY provides technical services to promote the use of available technology and to facilitate technological innovation in industry and Government; cooperates with public and private organizations leading to the development of technological standards (including mandatory safety standards), codes and methods of test; and provides technical advice and services to Government agencies upon request. The Institute consists of a Center for Building Technology and the following divisions and offices:

Engineering and Product Standards - Weights and Measures - Invention and Innovation - Product Evaluation Technology - Electronic Technology - Technical Analysis - Measurement Engineering - Structures, Materials, and Life Safety ${ }^{4}$ - Building Environment " - Technical Evaluation and Application " — Fire Technology.

THE INSTITUTE FOR COMPUTER SCIENCES AND TECHNOLOGY conducts research and provides technical services designed to aid Government agencies in improving cost effectiveness in the conduct of their programs through the selection, acquisition, and effective utilization of automatic data processing equipment; and serves as the principal focus within the executive branch for the development of Federal standards for automatic data processing equipment, techniques, and computer languages. The Institute consists of the following divisions:

Computer Services - Systems and Software - Computer Systems Engineering - Information Technology.

THE OFFICE FOR INFORMATION PROGRAMS promotes optimum dissemination and accessibility of scientific information generated within NBS and other agencies of the Federal Government; promotes the development of the National Standard Reference Data System and a system of information analysis centers dealing with the broader aspects of the National Measurement System; provides appropriate services to ensure that the NBS staff has optimum accessibility to the scientific information of the world. The Office consists of the following organizational units:

Office of Standard Reference Data - Office of Information Activities - Office of Technical Publications - Library - Office of International Relations.

${ }^{1}$ Headquarters and Laboratories at Gaithersburg, Maryland, unless otherwise noted; mailing address Washington, D.C. 20234.

${ }^{2}$ Part of the Center for Radiation Research.

${ }^{3}$ Located at Boulder, Colorado 80302 .

- Part of the Center for Building Technology. 


\section{A Technical Index of \\ Interactive Information Systems}

Dennis W. Fife, Kirk Rankin, Elizabeth Fong, Justin C. Walker, and Beatrice A. Marron

Systems and Software Division

Institute for Computer Science and Technology

National Bureau of Standards

Washington, D.C. 20234

Sponsored by the

Office of Science Information Service

National Science Foundation

18th and G Street, N.W.

Washington, D.C. 20550

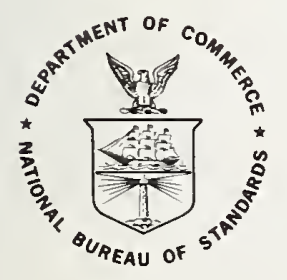

U.S. DEPARTMENT OF COMMERCE, Frederick B. Dent, Secretary

NATIONAL BUREAU OF STANDARDS, Richard W. Roberts, Director

Issued March 1974 
National Bureau of Standards Technical Note 819

Nat. Bur. Stand. (U.S.), Tech. Note 819, 73 pages (Mar. 1974)

CODEN: NBTNAE

For sale by the Superintendent of Documents, U.S. Government Printing Office, Washington, D.C. 20402 (Order by SD Catalog No. C13.46:819). Price $\$ 1.20$. 


\section{FOREWORD}

Despite a history marked by some significant failures and numerous partial successes, the field of computer software for information services now offers a wide and growing range of choices. Indeed, the variety and complexity of available software seems to overwhelm routine documentation and distribution as an effective means of dissemination. This report offers relief as a concise reference to technical features and operational status in the domain of interactive systems, i.e., those providing a "conversational" usage mode to a "non-programmer" through a data terminal device. The report was prepared under National Science Foundation support for NBS to assess the status of automated information services for scientific and technical information. (Grant NSF-CA68). The principal motivation for this report was a request for assistance from the COSATI Task Group on Interactive Systems, chaired by Mr. Alexander Hoshovsky, U. S. Department of Transportation.

We gratefully acknowledge the excellent cooperation of the system producers, the review assistance of the COSATI Task Group, and especially the support and thoughtful advice of Dr. Edward Weiss and Mr. Richard Lee of the Office of Science Information Service, NSF.

This report identifies computer systems by trade names as necessary to provide a descriptive characterization of their features. This in no case implies a recommendation or endorsement by the National Bureau of Standards, nor should the presentation be construed as a certification that any system provides the indicated capabilities. The information presented was primarily obtained from producer's documents and has been reviewed by each producer for accuracy and clarity, with the authors retaining final technical judgement on the information included. The report is only intended to be informative and instructive in state-ofthe-art assessments, and not to be a competitive evaluation of systems for any specific application. 
1. Introduction .................. I

2. Basic Characteristics of Systems Under Study . . . . . . 2

3. System Features and Evaluation Requirements . . . . . 5 3.1 Questionnaire with Explanatory Notes . . . . . . . 5

3.2 Suggested Procedure for Use . . . . . . . . . 5

4. Survey Results . . . . . . . . . . . . . . 14

4.1 Indexes to Systems .............. 14

4.2 The Inventory of Systems. . . . . . . . . . 17

\begin{tabular}{llll} 
Name & Page & \multicolumn{1}{c}{ Name } & $\frac{P}{\text { Page }}$ \\
\cline { 2 - 2 } & MARS VI & 41 \\
CDMS & 19 & MASTER CONTROL & 42 \\
CIRCOL & 20 & MICROTEXT & 43 \\
(Data/CentraI) & 21 & MINIDATA & 44 \\
DIALOG & 22 & MIRADS & 45 \\
DMARS & 23 & MUSE & 46 \\
DMI & 24 & NASIS & 47 \\
DRS & 25 & N.Y.TIMES & 48 \\
DS/3 & 26 & OLIVER & 49 \\
EMISARI & 27 & ORBIT III & 50 \\
ENFORM & 28 & PIRETS & 51 \\
FLEXIMIS & 29 & QUERY UPDATE & 52 \\
GIM & 30 & RAMIS & 53 \\
GIPSY & 31 & RECON & 54 \\
IMARS & 32 & RFI & 55 \\
IMS (OEP) & 33 & RIQS & 56 \\
IMS /360 & 34 & SHOEBOX & 57 \\
IMS / & 35 & SOLAR & 58 \\
INQUIRE & 36 & SPIRES II & 59 \\
INSYIE & 37 & STAIRS & 60 \\
LEADERMART & 38 & SYSTEM 2000 & 61 \\
MARK IV & 39 & TICON & 62 \\
MARS III & 40 & UNIDATA & 63
\end{tabular}

5. Glossary of Conmon Data Bases. . . . . . . . . . . 64

6. Bibliography . . . . . . . . . . . 68 


\section{A TECHINICAL INDEX OF INTERACTIVE INFORMATION SYSTEMS}

Dennis W. Fife, Kink Rankin, Elizabeth Fong Justin C. Walker, Beatrice A. Marron

This report constitutes a reference to technical features and operational status of interactive information systems, i.e. those providing a 'conversational' usage mode to a 'non-programmer' through a data terminal device. It is aimed at the ADP service manager, for his use in the state-of-the-art assessments preparatory to a detailed system selection process. It contains an index that describes 46 systems in terms of a list of over 50 technical features plus descriptive, identification, and background information. In addition, there are aids and examples contributing to the intended use of the index.

Key words: Bibliographic systems; computer programs; computer systems; data base; data management; information retrieval; information services; interactive system; query language; software selection; text processing.

1. INTRODUCTION

This report is written for the purpose of providing Federal ADP customers with information on a certain class of computer systems which are capable of handling scientific and technical information. The report attempts to show what is available and to characterize these systems in such a way as to answer questions which naturally arise prior to selecting such a system for a particular installation. The report is written at a level of technical detail which is aimed at information specialists rather than programming experts. It is intended to be informative and instructive, and not critical or evaluative.

We have reviewed for inclusion in this index over 200 systems which came to our attention from various published and unpublished sources as well as from word-of-mouth. The systens which were selected conform to the following definition: "Information Retrieval" or "Data Management" packages or services which are available to any Federal ADP installation, and which offer an interactive query and search capability that is geared for use by non-programmers. Various reasons for rejecting the systems which did not qualify for inclusion are listed at the end of Section 2 of this report.

The information contained here on each of the systems was obtained primarily from documentation received during 1972 and 1973. In cases where the available documentation did not provide sufficient data, further information was obtained by telephone. We believe that the reporting on these systems is accurate, for it has been, reviewed and amended where necessary by the system producers. We regret if this report inadvertently omits any system which may meet the definition. Omissions and out-of-date information are inevitable in a survey of such an extremely active and evolutionary field as this. 
A caveat on the use of this index must be mentioned. We have attempted to convey in a limited space information obtained from secondary sources. There are obvious limitations in such a treatment. The best way to gain in-depth familiarity with a system is, of course, to have hands-on experience with it. That has not been possible for most of our treatments, but that course is recommended to readers and users of this inventory.

This report is structured as follows: Section 2 elaborates on the definition of the scope of the survey and clarifies some of the important concepts and sometimes confusing terminology. Section 3 discusses evaluation for system selection, so that a manager can relate his needs to technical features which appear in oun questionnaire. Section 4 presents the technical data on systems surveyed. Section 5 presents a brief description of the widely known, commercial data bases that are accessible on some of the surveyed systems. Section 6 presents a bibliography of related work that may also be helpful for information sources.

\section{BASIC CHARACTERISTICS OF SYSTEMS UNDER STUDY}

In this part, we discuss the rationale for including certain systems in this index and excluding others. The intention has been to include every system for which there are "yes" answers to the following questions:

(1) Does it have an interactive query and search capability?

(2) Is this capability geared for use by non-programmers?

(3) Is the system available to any Federal ADP customer?

We will now explain what is meant by the three questions. The first question is dealt with extensively, since arriving at an acceptable understanding of "interactive" has proved to be quite difficult.

\subsection{Interactive Query and Search}

The term "interactive system" is elusive when attempts are made to pin down an exact definition. The problem is complicated by the fact that this and related terms have been used for promotional and advertising reasons as much as for technical reasons. Rather than try to establish a hard definition, we sketch the intuitive meaning for the term by placing it in perspective with other terms.

Terms currently in use to describe the processing of jobs by a computer system are batch, remote batch*, on-line, and interactive. Intuitively, batch processing requires that all steps in a job, together with any required input, be specified prion to the job's initiation. A dis-

\footnotetext{
"Remote job entry" or "RJE" is often used and is synonymous with "remote batch".
} 
tinction can be drawn between local batch and remote batch systems according to input via direct lines on via communication lines. The latter require an extra layer of protocol which sometimes must be specified by the user. A batch program is one in which all parameters are specified in advance, but also whose results are known only after completion of the whole job.

The term "on-line processing' is generally used to describe a spectrum of processing modes. The user communicates with an on-line system via a typewriter-like terminal (i.e., a terminal with a keyboand and possibly a display). The system actively acknowledges user input in some form, as opposed to the more passive remote batch systems. The spectrum of processing modes is determined by the extent of this acknowledgement, e.g. prompting after receipt of "input lines", prompting plus :-rntax cheak of control cards, and so on. Interactive systems form a portion of the on-line spectrum. They are characterized by "friendly" responses to input errors, relatively rapid response to system commands

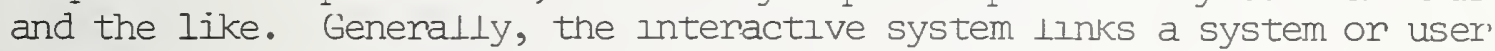
program directly with the user at all times, in contradistinction to other on-line systems. In the latter type, user and systems programs utilize the terminal mainly as a line printer.

The above distinctions are not rigorous, and from the user's view may be non-existent in a fast-response environment. The following discussion tries to focus on the "interactive" system from the user's point of view. In the survey we include systems from the entire on-line spectrum but we have attempted to restrict inclusion to tiose which present some indication of interactivity either from the above consideration or from those following.

Imagine a system which allows a user to formulate a query at his terminal and submit that query for searching against a file, and which prints out the records which satisfy that query, within a matter of seconds. Such a system qualifies as having an "interactive query and search capability", although it is at the weaker end of the spectrum compared to a system as now described.

This system allows the user to incrementally formulate a query, and responds with intermediate results (e.g. the number of records satisfying the query or similar terms or phrases which the user may also consider), based on which the user can re-formulate or extend his query. This cycle of system response with intermediate results leading to user re-formulation of query, etc. need have no upper limit. For example, the following user/system session is typical of one of the major applications of interactive systems, namely bibliographic search.

The user enters his query, e.g. "computers", which is checked for inclusion in an index of permitted terms. It is found to be included and found to be a descriptor of say 1,104 documents in the system. The system responds with "1,104 hits". At this point the user modifies his original request with "and the United States". The system responds with "52 hits". The user then modifies his now complex query with 
"published since 1971". The system responds with "'6 hits". The user requests the titles of these documents. On viewing them he then requests the full citations of three documents from that six that he really is interested in. Such a system would be at the stronger end of the "interactive" spectrum.

\subsection{Use by Non-programmers}

Many customers want the capability of having a user who is not a programmer formulate queries at a terminal in a way that is relatively natural for him. Many systems are designed in such a way that their interactive query and search components are geared for use by non-programmers: information specialists, managers, or clerical personnel. We have attempted to restrict our survey to such systems.

In some cases, the documentation for a system states that it is for use by programmers. All such systems have been rejected. In some cases not so rejected, inspection of a system's documentation forces us to reject it because there are requirements on the user to "do programming" of a sort. By this we mean that the user has to phrase some or all of his query (or operations preceding the query) in language constructs which are primitive and unnatural in relation to his task of query and search.

For example, one system defines its files in terms of physical storage structure. In querying files, the affected files must be described by such parameters as transport number, number of logical records per physical record, and address of first block (of tape) or sector (of disc).

Another system has essentially a high level programing language for querying files. Each query is bracketed by a BEGIN-END pair and language statements within are numbered hierarchically. Each statement potentially produces a result which can be referred to in other statements by giving the statement number. An example is:

BEGIN

1. FIND ALL RECORDS FOR WHICH GRADE $=2$

2. COUNT RECORDS IN 1

3. PRINT ... COUNT IN 2

END

The primitive nature of the statements forces the user to construct a program to query the system, even for the most logically simple requests.

\subsection{Availability}

We are including in this index systems which are available to the Federal customer. Availability implies that the system is operational 
(not in research or development) and still supported. There is also an understanding that there are no legal, security, or other problems in the way of availability. The systems included here are either available on a commercial basis or on a less formal and less costly basis in cases where the originator is not a commercial establishment. Further, we have included both those systems which are available on a service basis and those which are available as packages for lease or purchase.

To summarize, we have attempted to include everry data management or information retrieval system for which there are "yes" answers to the three questions. Reasons for not including a system are: (1) it is a batch system; (2) its query language is not for use by non-programmers, (3) it (or its query language) is in research or development; (4) it is no longer supported; (5) the originator of the system is unlocatable or no longer in business; (6) there are legal or security problems in the way of releasing the system; and (7) documentation has been solicited but not yet received.

\section{SYSTEM FEATURES AND EVALUATION REQUIREMENTS}

\subsection{Questionnaire with Explanatory Notes}

Systems are built with different objectives and hence have different capabilities for different application situations. Since not all systems provide the same set of features to the same degree, it has been necessary here to establish features for describing systems relative to a broad range of possible user needs. The feature list or questionnaire used in this index is therefore the union of a wide number of possibly relevant capabilities, see Table 1. A specific system is characterized by a subset of the features covered.

\subsection{Suggested Procedure for Use}

The survey results, Section 4, represent a nearly complete inventory of available interactive software, and therefore are a basis for assessing the state-of-the-art in this field. The index is intended also to be informative in the preliminary, exploratory stage of a system selection activity. In this area, the index may aid a manager in articulating his requirements using the offered features as a starting point. Further, the index may be useful in forming a tentative set of candidate systems, by screening the inventory against a required subset of features. The latter of course imposes an inescapable burden on the user to relate his unique needs to applicable features.

The suggested procedure for using this index can be illustrated by briefly discussing questions arising in formulating requirements and evaluating information systems. Two basic alternatives would face an information service manager once he has decided to investigate the feasibility of automated service, see Figure 1 . If the expected usage is very high and a computer facility is in place, an in-house service could be considered. Compatibility with the available mainframe may 

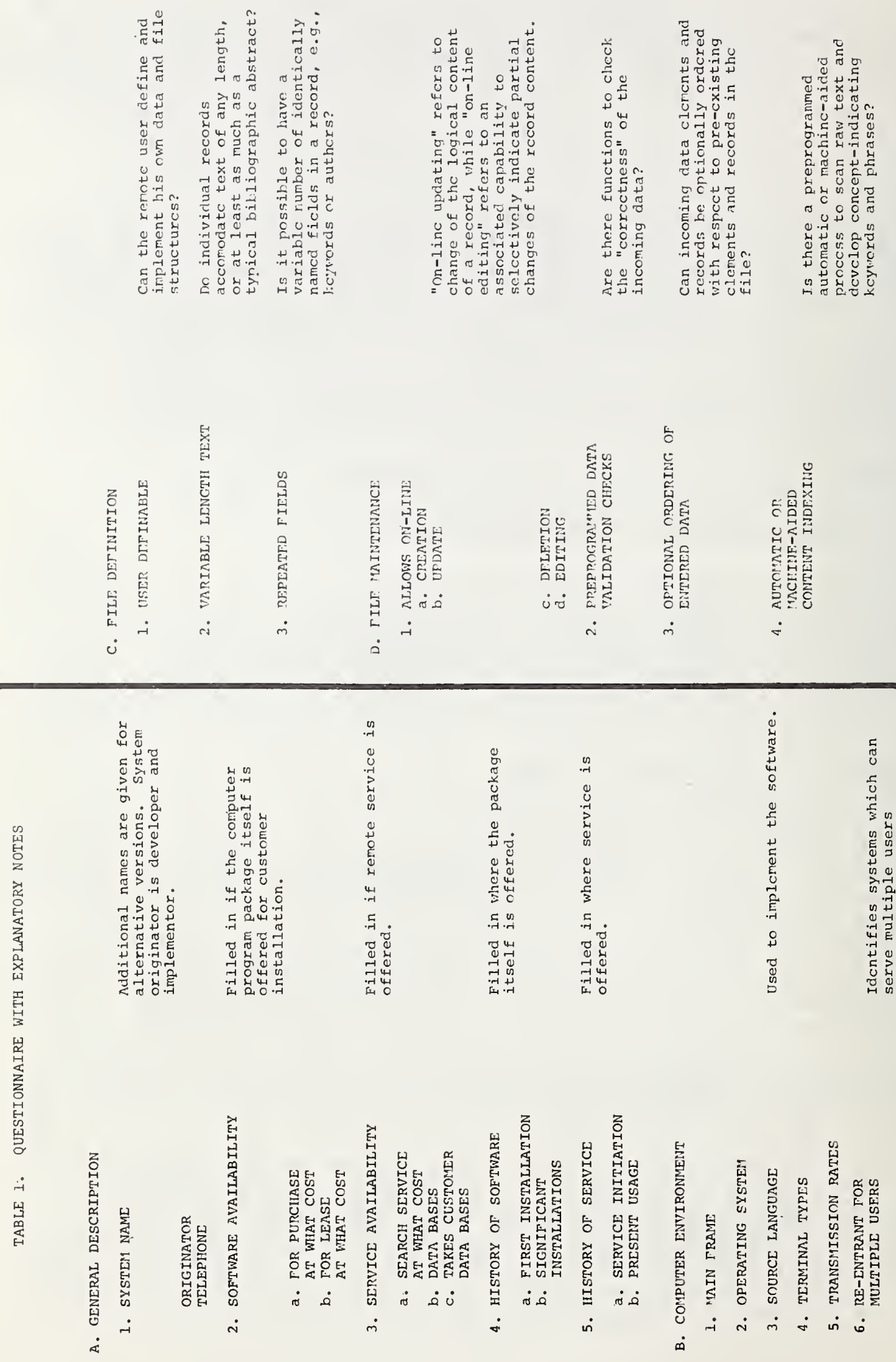


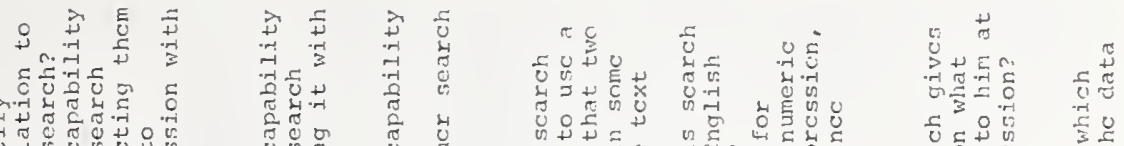

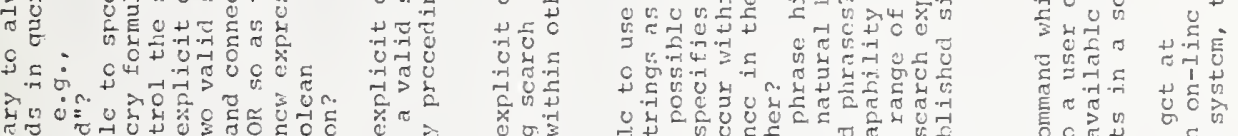 率o

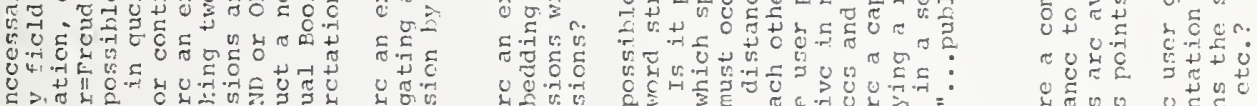

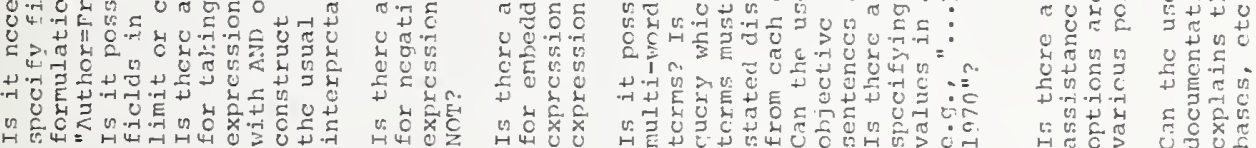

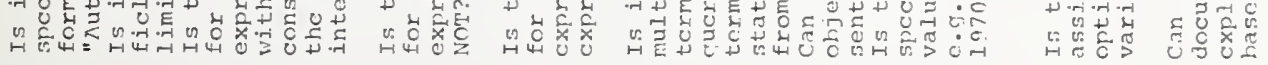

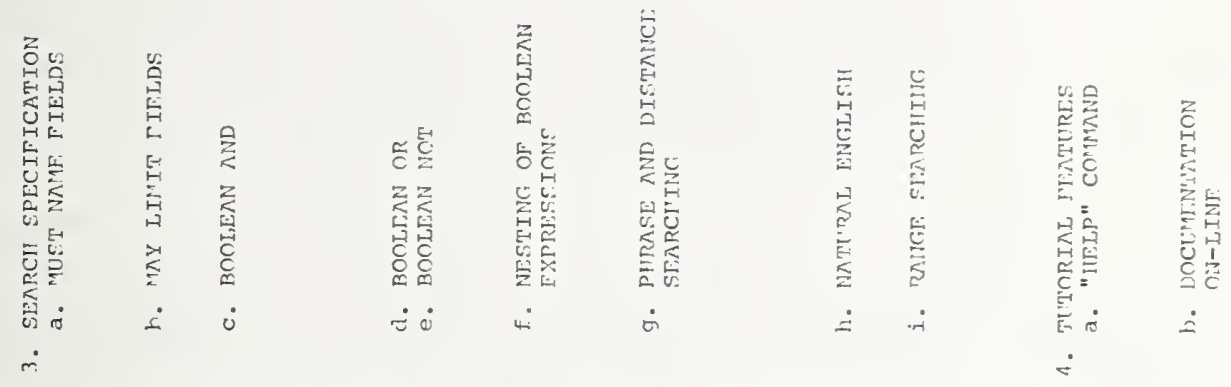

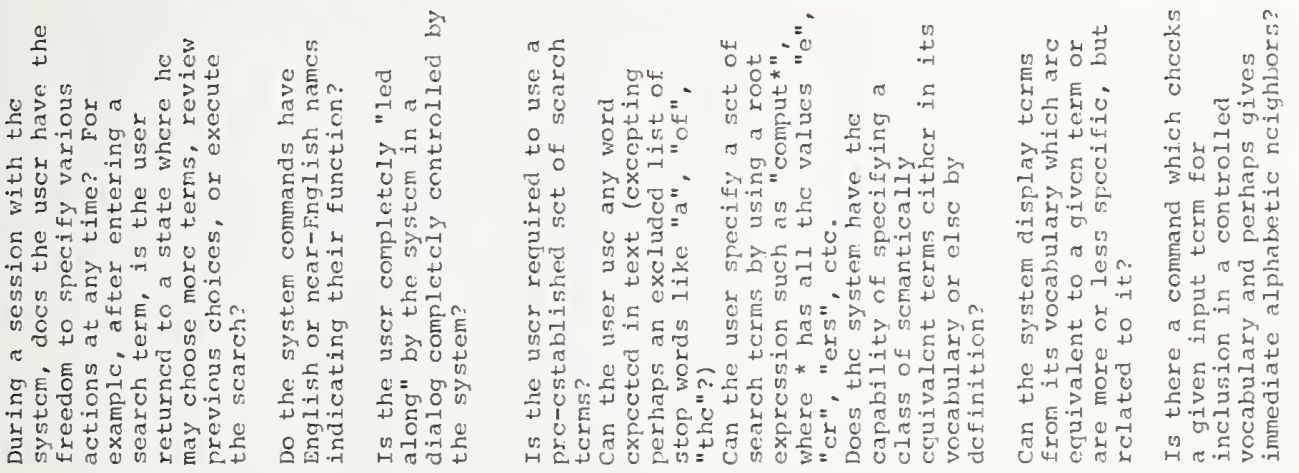

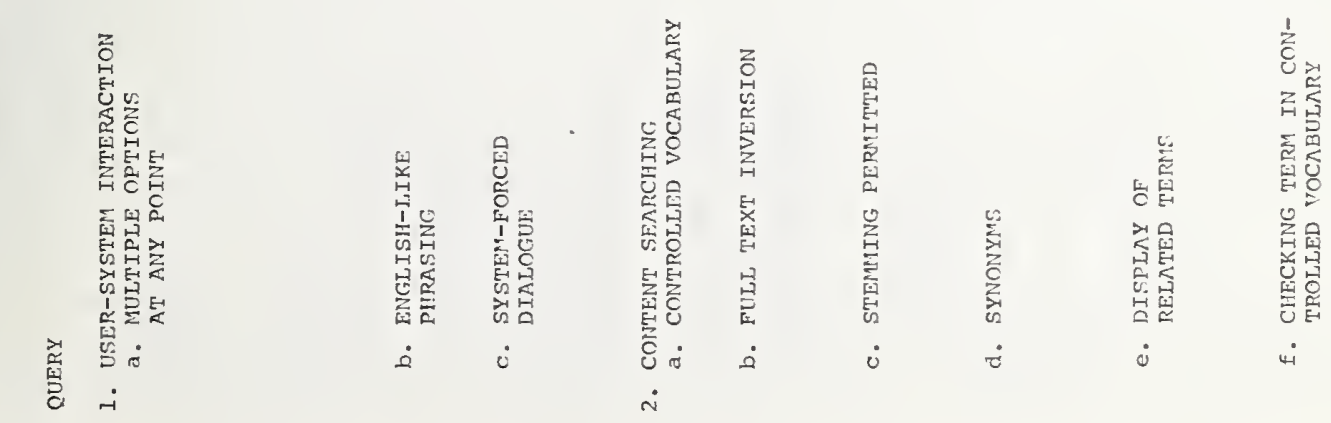



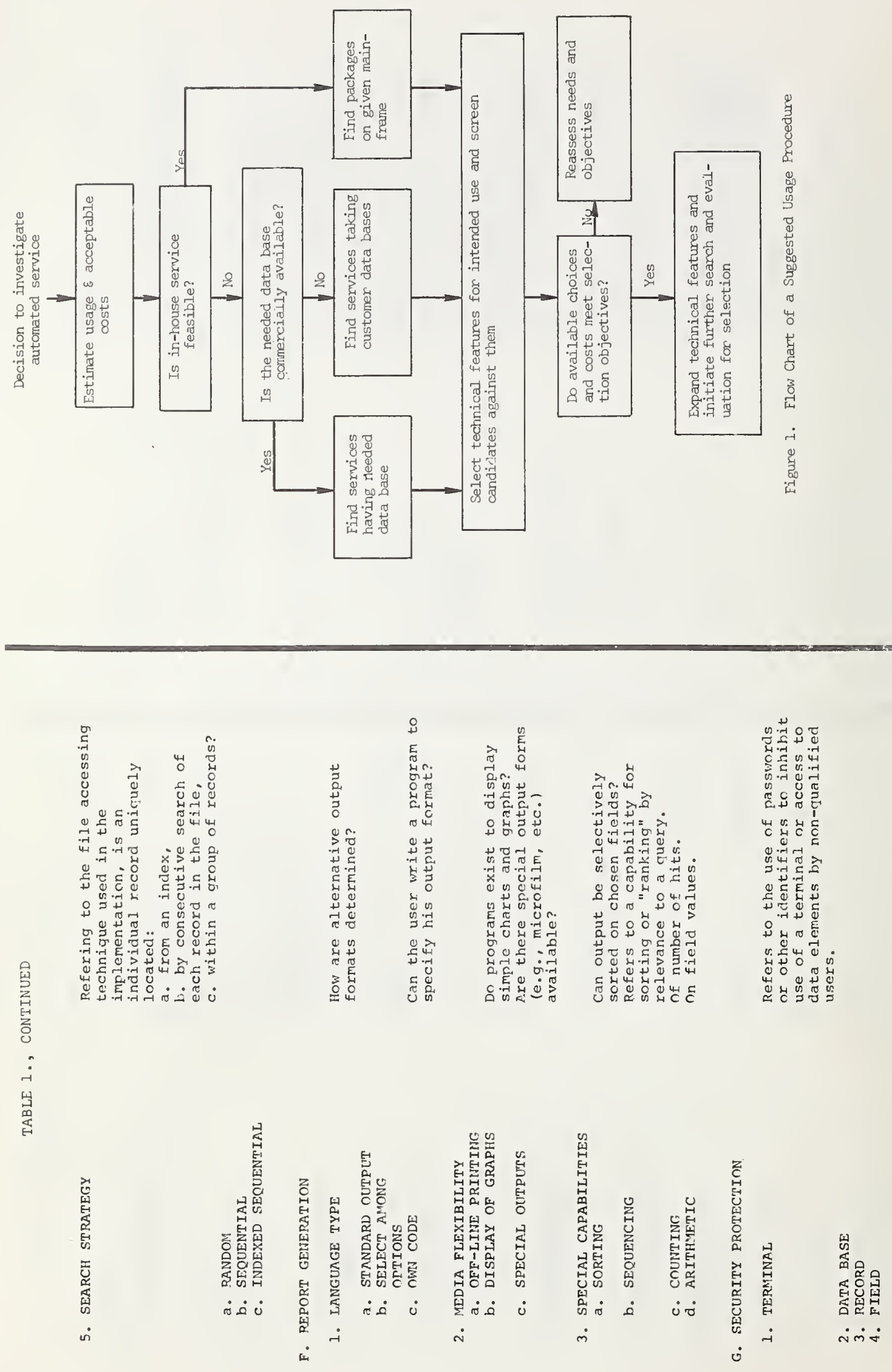
then be the overriding factor in the selection of a software package to be installed. The index can be used to find as prime candidates the systems implemented on the given vendor hardware. However, if expected usage is low, say on the order of a few hours of terminal use per day, or if a computer center and necessary expertise are unavailable, a worthy alternative would be to obtain use of software as a service from an outside computing center. A pertinent question then is whether the service offers searching of a major commercial data base, if required, or takes customer data bases if a special private file is involved. The index also assists in finding initial candidates based on these factors.

Having pursued one of the above altematives to identify a relevant subset of available systems, attention should turn to technical features desirable or necessary for the intended application. The questions in the index were purposely selected to draw out distinctions in three broad classes of system application, called respectively formatted data processing, structured text searching, and personal text handling. As a tutorial guide for the index user to articulate his unique requirements, these classes will be individually discussed toward framing a representative list of essential and additional desirable features (Tables 2-4).

Formatted data processing is conceived as most typical of management information, military command/control, and other applications where data elements have mostly numerical, abbreviated text, or other encoded (e.g. date) values. Personnel, inventory, or production control files would be comnon examples. Data values in the file may need to be updated frequently as status changes occur in the application environment (e.g. personnel are reassigned, parts are used, or products moved from one manufacturing station to another). This is motivation for requiring on-line file maintenance as an essential capability. In Table 2, therefore, D. 1 indicates that a qualifying system must allow update or editing transactions to be entered from the user's remote terminal (see Table I for explanation of items under D.I). Formatted data processing software must provide considerable flexibility to the user in manipulating file data in any logically correct order and with a wide range of arithmetic functions. These are essential because such systems are usually appliec concurrently to a number of differently organized files. Also, they must help their user to answer an indeterminable variety of questions about relationships and quantities in the file data. In Table 2 , the conclusion is reflected in choices of: an interaction language with mltiple options (E.l.a), a full Boolean capability to express logical or comparison conditions (E.3.C through E.3.f), and arithmetic capabilities (F.3.C and d). Users of these systems also require a range of options in formatting output reports, so that essential factors can be made clearly visible and effectively ordered with respect to related data. For these reasons, optional formatting (F.I.b and C) and ordering (F.3.a) are included as essential in Table 2.

Related to these essential features are several additional ones that seem especially desirable and are often available. In some circumstances they may be considered essential. Repeatable fields (C.3) 
are a great convenience in organizing and referencing the machine file when records have multiple occurrences of the same type of data (e.g. children or work experience in a personnel file). This may also save considerable machine storage for many files. English-like language phrasing (E.I.b) aids user comprehension and ease of use, in conjunction with the multiple option language characteristic. Range searching (E.3.i) and displays of graphs (F.2.b) significantly enhance selection of desired records and data presentation in a more readily perceived form. Tutorial aids (E.4.a or b) are highly desirable, even essential, to bring users quickly toward effective use of complex software or to help solve usage problems as they are encountered.

Structured text searching (Table 3) is conceived as representative of bibliographic information searching, legal text searching, and similar uses where the file records consist of prescribed segments of text, individually of variable length (C.2 in Table 1), possibly rather long (1000 characters or more). Examples of text segments would be report titles, abstracts, patent claims, paragraphs, statute sections. To identify a text record for selection there must be a technique for abbreviated content description, since requiring an exact match to all the text in a segment would be inconceivable and inconsistent with the intended function. Content description may be provided by indexing each file record by a set of keywords from a controlled vocabulary (E.2.a) which the user can inspect to check his desired term for acceptability (E.2.f). Or else, any significant word occurring in text may be provided as a valid search term (E.2.b). Because these systems are especially aimed at users .unaccustomed to programing encoded forms, Fnglishlike phrasing is deemed essential as well. 'Because a search may select voluminous text records that would be exceedingly long to print on the usual 10 or 30 character/second terminals, off-line printing (F.2.a) at high speed is also essential. Moreover, these systems should present a count (F.3.c) of the records that would be selected by a proposed search so that a user can judge the desirability of continuing the search.

The data files of structured text searching systems would be expected to be unchanging in content and very large in volume. It would be expensive to reorder or restructure them is new data is received, so it would be desirable for the system to accept new data in any order (D. 3). Other desirable features would extend content searching capability, for example by giving a synonym facility (E.2.d) or a presentation of other terms that are conceptually related (E.2.e). As in formatted data processing, tutorial aid is desirable. In contrast to that application however, full Boolean capability, optional report formatting, and optional ordering are suggested here as desirable rather than essential. Only a Boolean AND, allowing the conjunction of distinct search terms, is imperative for user convenience, to avoid a tedious selection from record subsets found by individual terms. Optional formatting and ordering may not be used often for such simple structured output records as bibliographic citations. A standard output presentation then is generally sufficient, unless text fields become numerous and frequently of marginal importance, requiring more selectivity to be given the user. 
Personal text handling is conceived as a generalization of structured text searching to uniquely serve an individual researcher in creation, editing, and composition of text bodies as well as searching them. Examples arise in military intelligence work, legal case preparations, source book or encyclopedia preparation, etc. Most of the requirements of structured text searching apply. The most germane additions are for user definable file stmuctures (C.I) and on-line file maintenance (D.I), which allow complete user flexibility to establish arbitrary new files in the course of his interactive usage. Optional output formatting becomes essential because of the potential variety and scope of file structures. A highly desirable search capability would be phrase and distance searching (E.3.g), to permit the researcher to more narrowly select desired reconds based on occurrence of phrases or words in context (separated by linited distance). As the researcher may establish many records with grossly similar content within his special interest, this additional selectivity has great importance.

The above rationale indicates the type of analysis which may lead the user to a pertinent feature list for his situation.

Table 5 illustrates using the worksheet for structured text searching to develop comparative presentations of system capabilities. These are the basic questions which the index user may answer in such fashion: are there systems offering the essential combination of features? is there a good number of desirable systems, portending a competitive choice in cost, types of vendor support, proven system reliability, etc.? is the cost range of desirable systems within the funds available for a purchase or subscription? Positive answers should build confidence that an economical, proven system can be obtained, while negative answers may indicate a presently infeasible set of requirements or a constrained area where further research and system development is advisable.

It is suggested that each index user develop his own worksheet of features for his needs. Table 1 shows that the index permits a sharper delineation of required features than used in Tables 2 to 5 . It is expected also that individual readers will perceive special features for their need that are not used here, and will have to be specially investigated for all systems of interest. The index is meant to help draw attention to that subset of systems of greatest interest and relevance.

The index is primarily meant however to aid state-of-the-art assessments. We see in the index results many disparities calling attention to needed research, and also many common factors indicating a readily available, recognized feature. Reganding the former, we note particularly that only half of the systems have tutorial capability - yet none are considered trivial to use. The design of effective training aids and problem diagnosis features is a very important area for further development. On the other hand, almost all systems offer English-like phrasing in their language style. This is encouraging for it is clearly desirable and of little added cost to implement in terms of meaningful command names. 

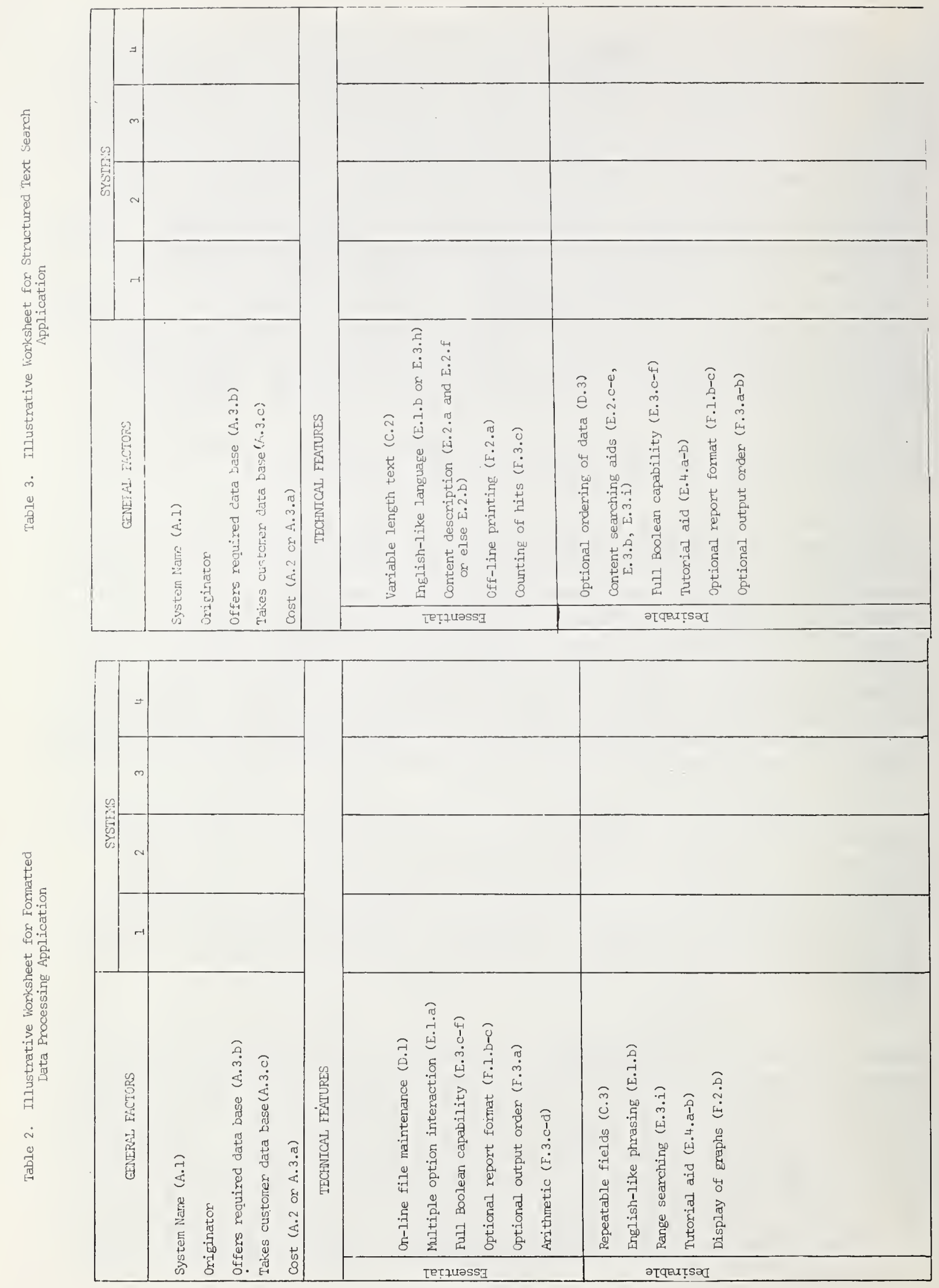


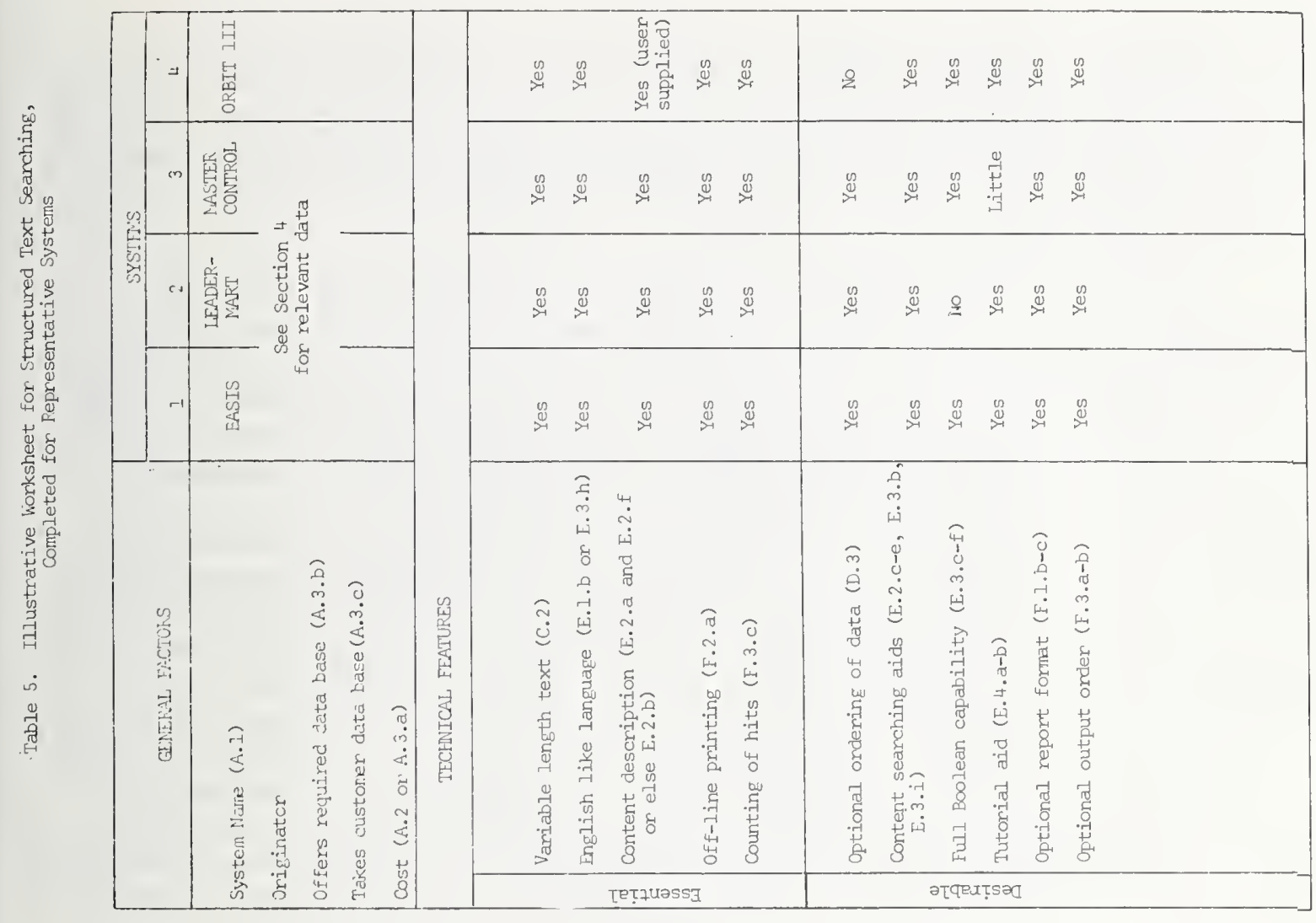

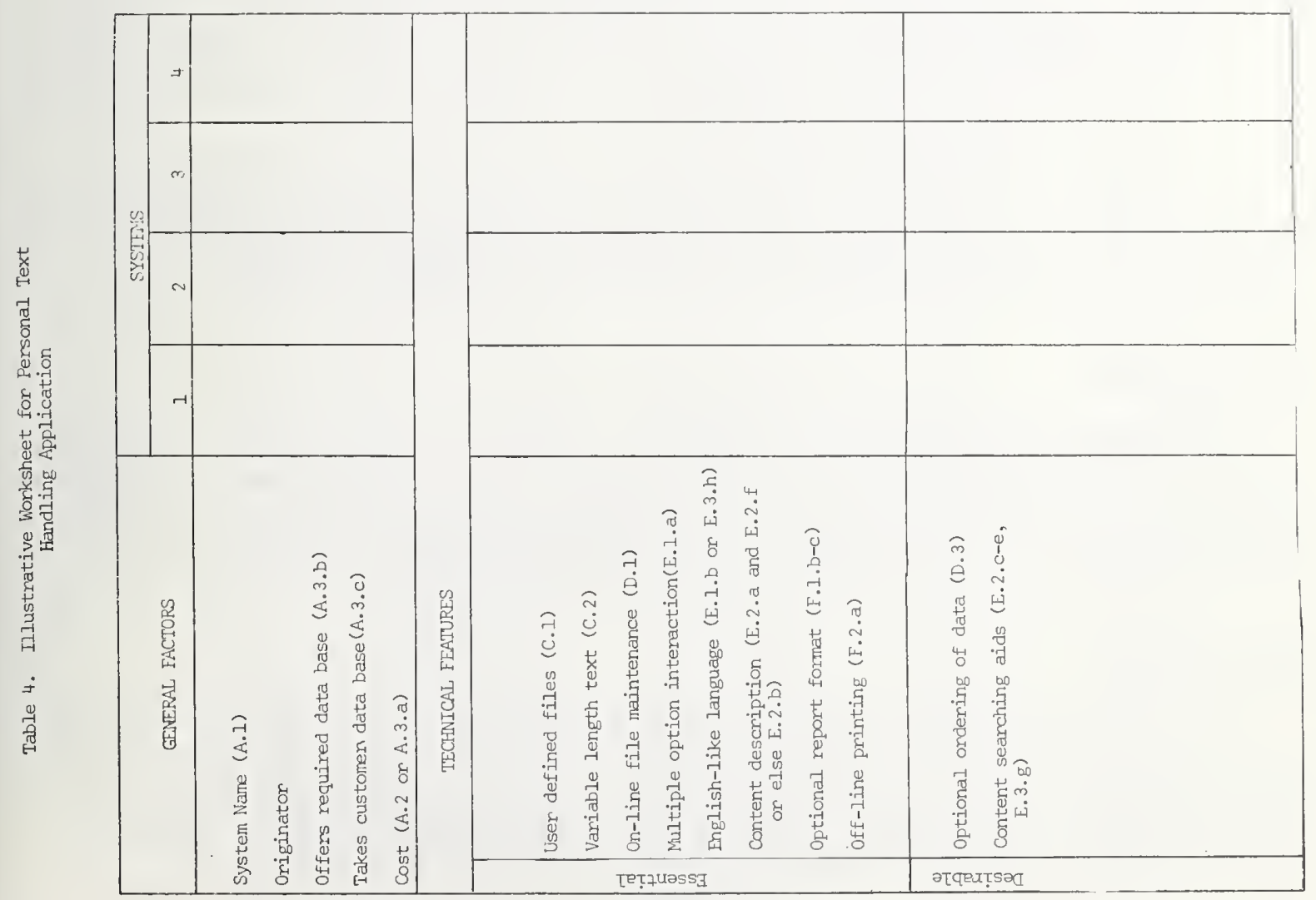


Section 4 presents several matrices that illustrate how the features may be used individually and in combination to categorize systems as may be relevant to user needs. It is advisable however for a user to create his own worksheet of pertinent features and draw information from the survey, e.g. Table 5, to judge the capabilities that are available in the field.

It is neither intended or recommended that the survey results or the broad feature list should be adopted as the exclusive basis of a system selection. A competitive selection decision requires a much more detailed matching of user requirements with functional features, coupled with a productivity assessment addressing performance and cost factors. The background for this task necessarily involves familiarity with a user's organization and its forcing constraints, such as the needed data files and their characteristics, funds available, allowable implementation time, present computer equipment, and available programming skills. The tools to be employed in system evaluation include benchmark exercises, performance monitoring, simulation and modeling, hands-on experience, detailed documentation review, and thorough audit tests. Inasmuch as this survey has been based primarily on documentation review, it cannot be evaluative of one system against another. Managers facing a selection decision are advised then to acquire system documentation, at least to the level of a complete user's manual, and to undertake hands-on familiarization or benchmark exercises to thoroughly understand each system's capability for his user community and procurement constraints.

\section{SURVEY RESULTS}

\subsection{Indexes To Systems}

As an aid to the reader, this section presents several examples of classification of information systems drawn from the questionnaires. Following is a brief description of each of the four illustrative classifications.

\section{Applications:}

As examples of application classes, there are three types considered: formatted data, stmuctured text and personal text. The relevant features have been described in Section 3.2. Table 6 presents a matrix for this categorization. This may help the reader direct his first attention to systems closely corresponding to an intended usage.

\section{Main Frames:}

The matrix of Table 6 dealing with mainframes correlates the systems in the survey with the (classes of) main frames on which each runs. The classification "other" is used for those main frames for which only one of the systems is available. For these systems, reference to the specific questionnaire will give the appropriate main frame (B.1). 

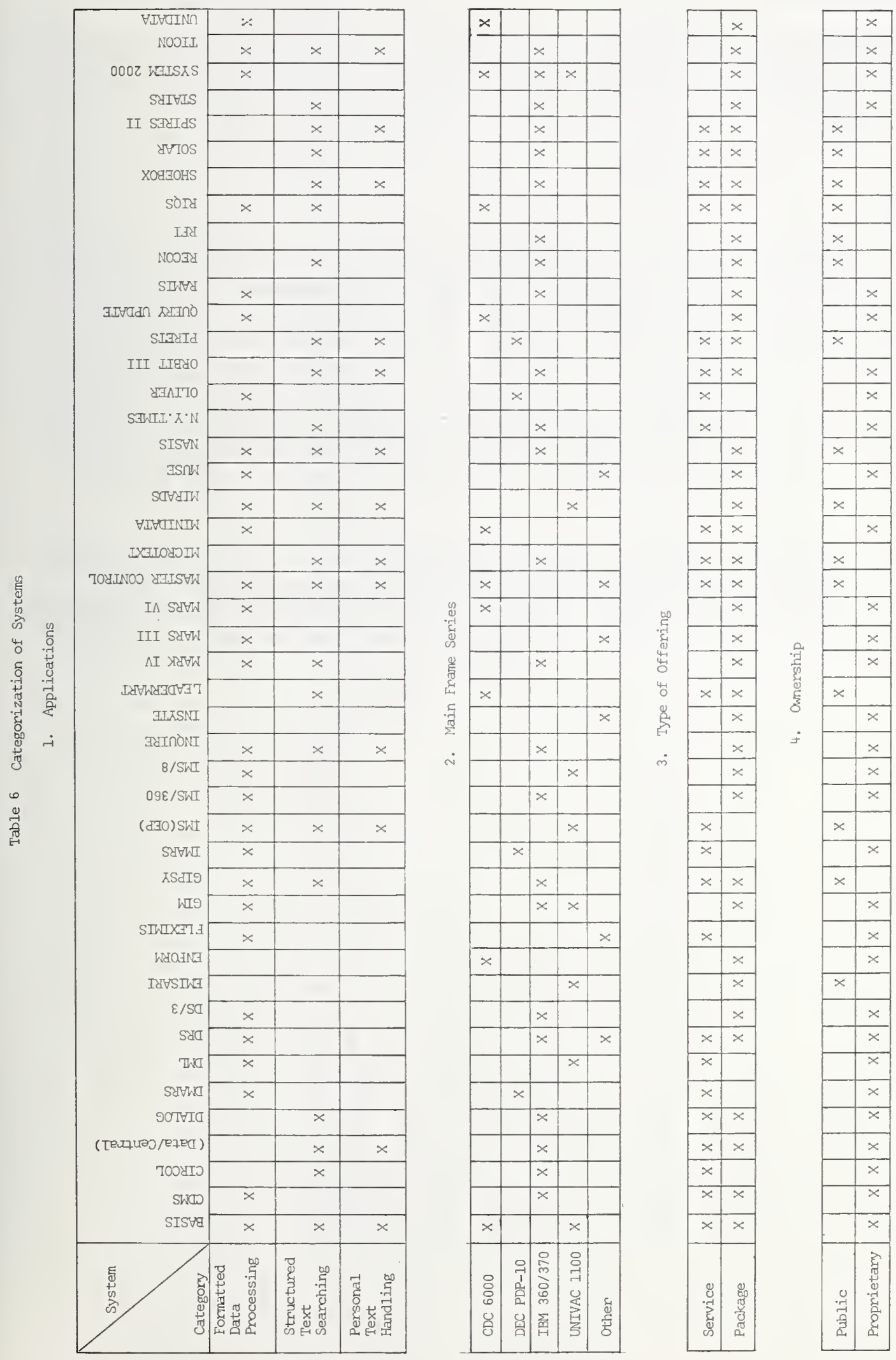


\section{Type of Offering:}

Questions A.2, 3 deal with the availability of a system and in particular answer whether the system is available as a software vendor's package to be installed at the user's site, or as a service running at the vendor's site.

\section{Ownership:}

Two categories are used to describe ownership of a system. The term "public" designates those systems in which the originator (or vendor) has not retained a substantial monetary interest, such as those which have been completely government funded. These systems are generally available (source and source listings) for a nominal fee, such as tape duplication. In some cases, the producer or vendor may only consider assistance for a fee, covering installation, modifications, and possibly maintenance. This assistance may be contractual and include on-site personnel in the case of packages, or resource costs, in the case of services.

The "proprietary" systems are those that are wholly owned by private concerns. Generally there is a purchase price on the system. However in the case of some main frame manufacturers, the system may be available without additional cost to the purchasers on lessors of their equipment. 
4.2 The Inventory of Systems

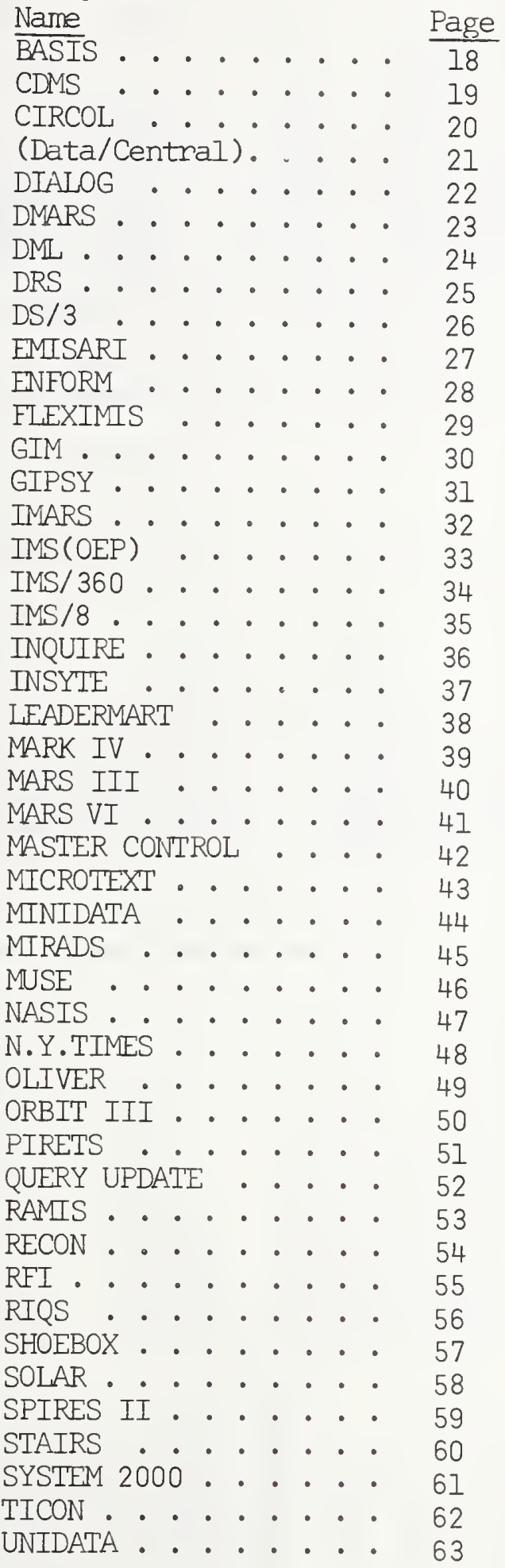




\section{解}

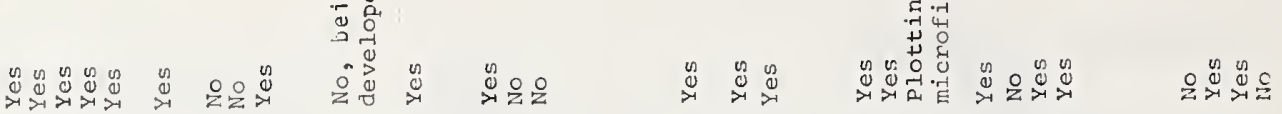

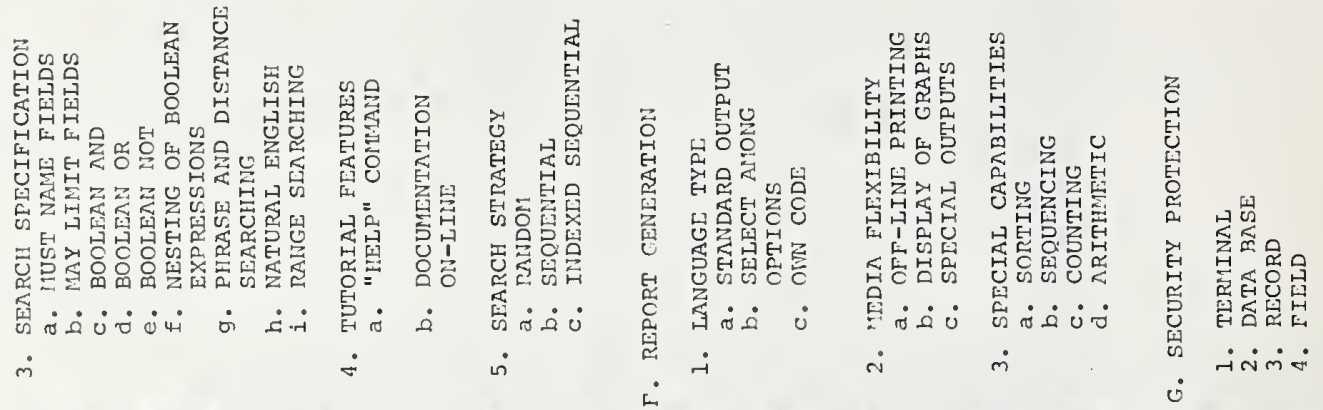

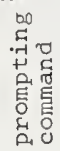

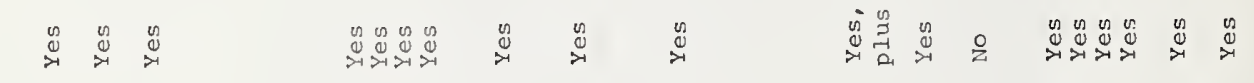

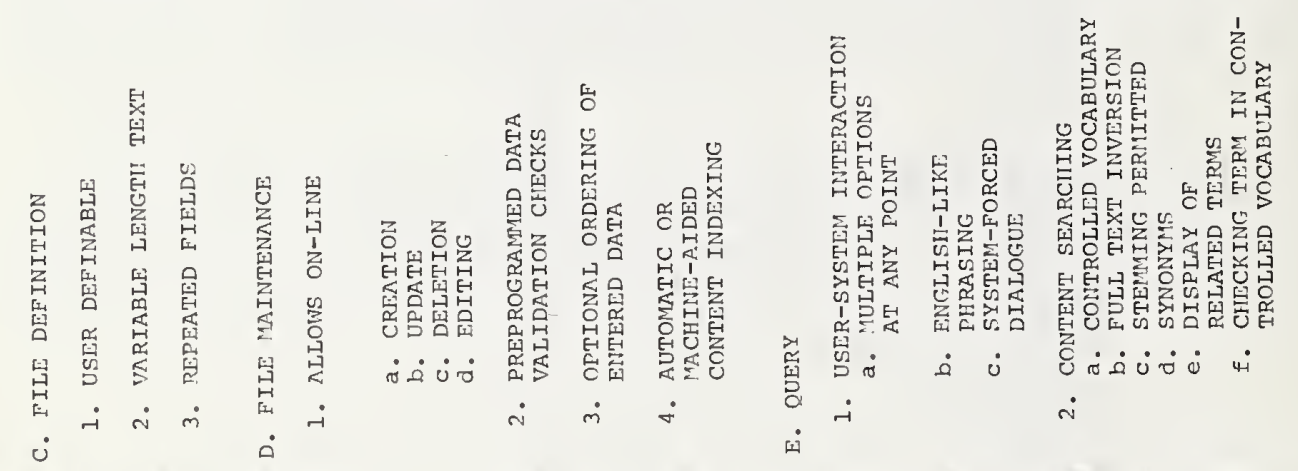

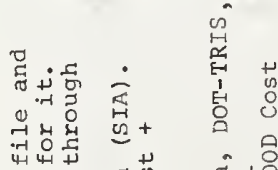

蹈

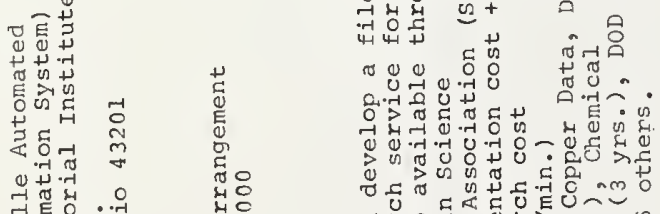

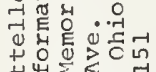

ํㅐㅇㅇำ

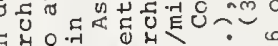

तै

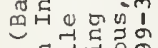

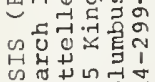

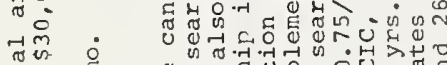

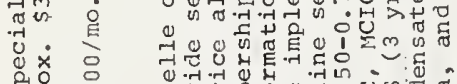

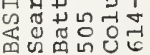

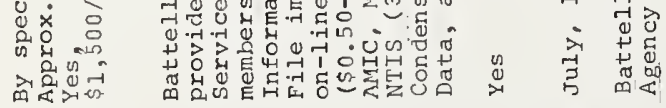

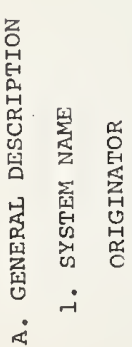

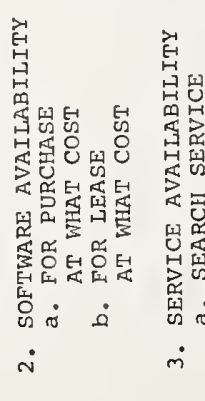
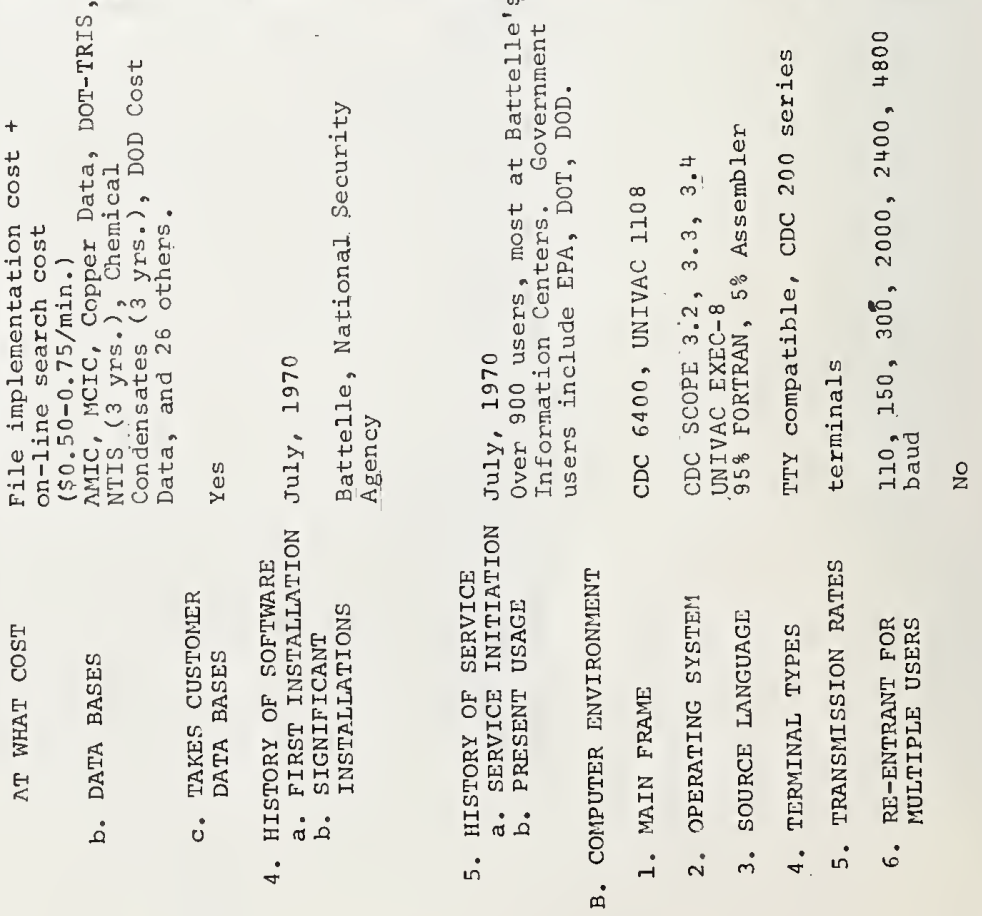


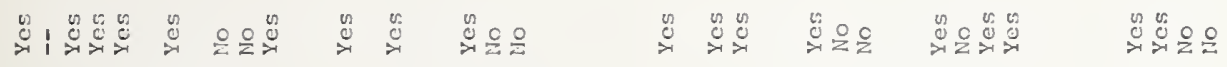

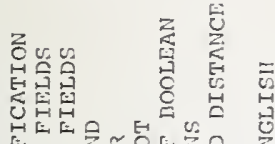

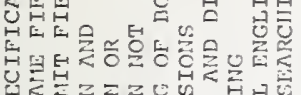

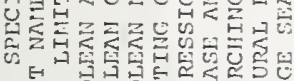

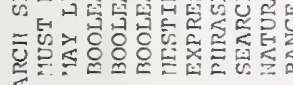

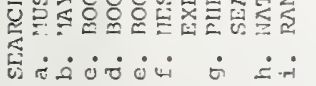
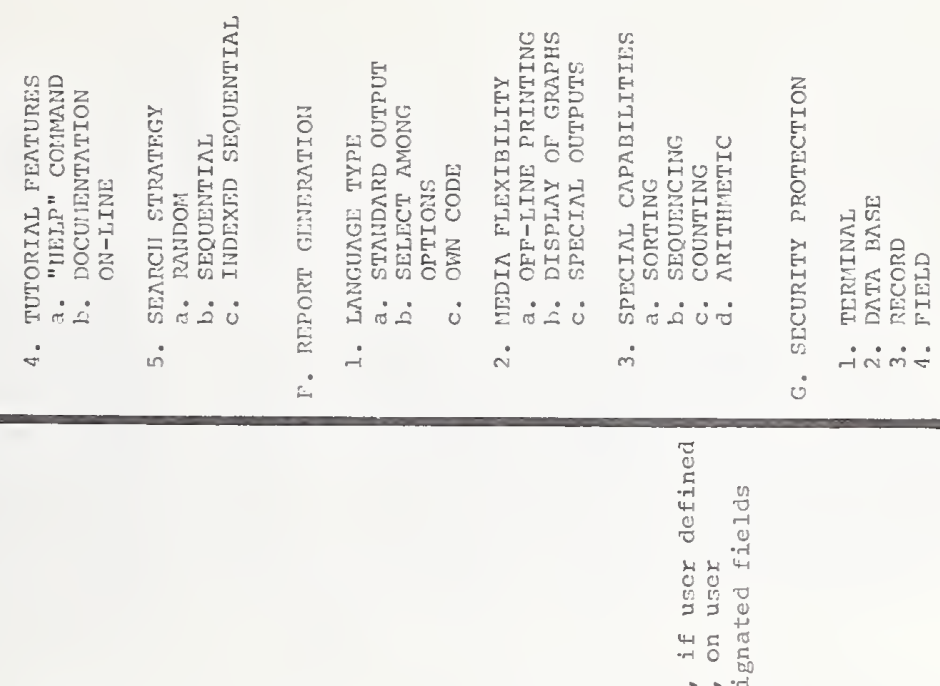

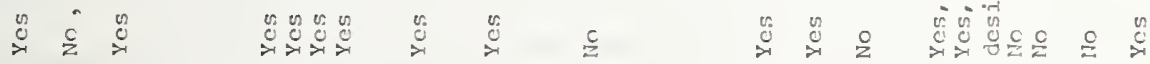

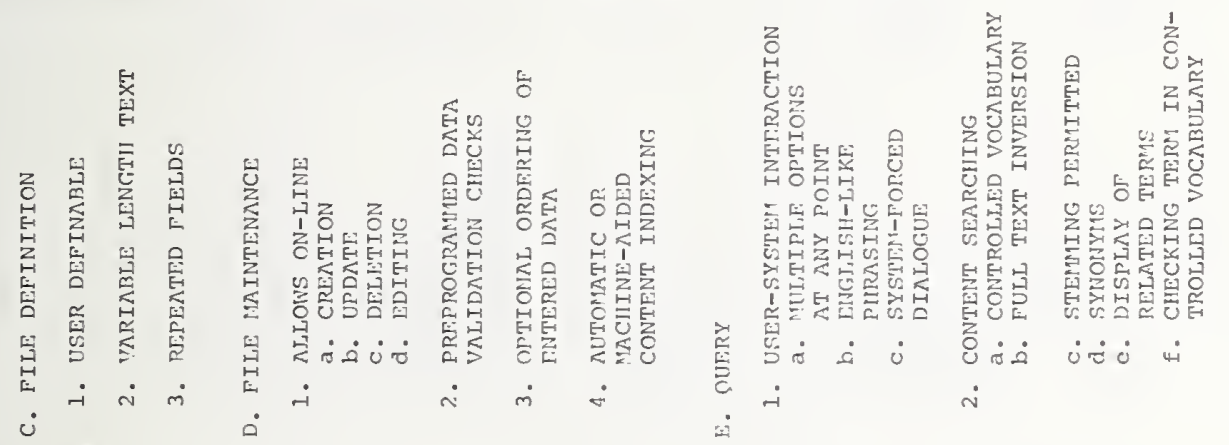

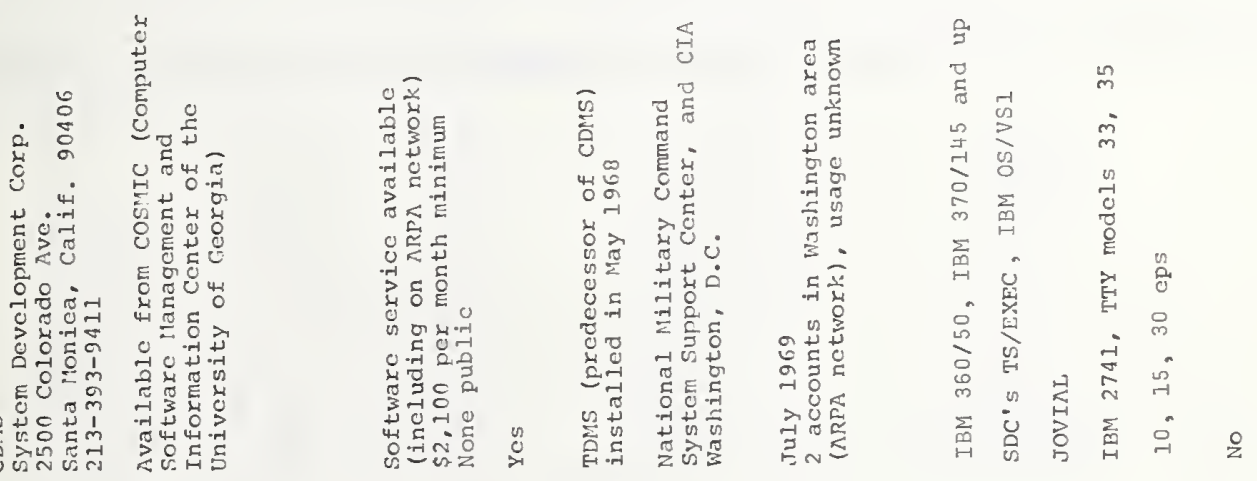

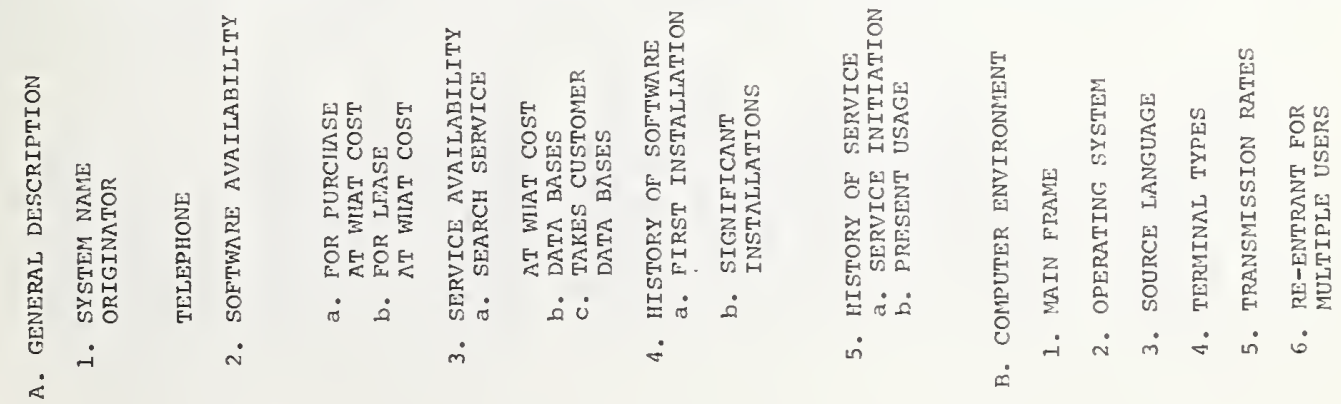




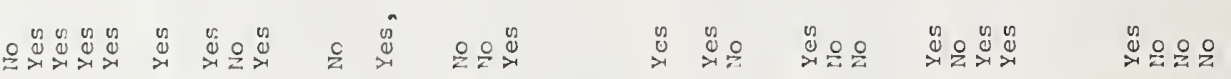

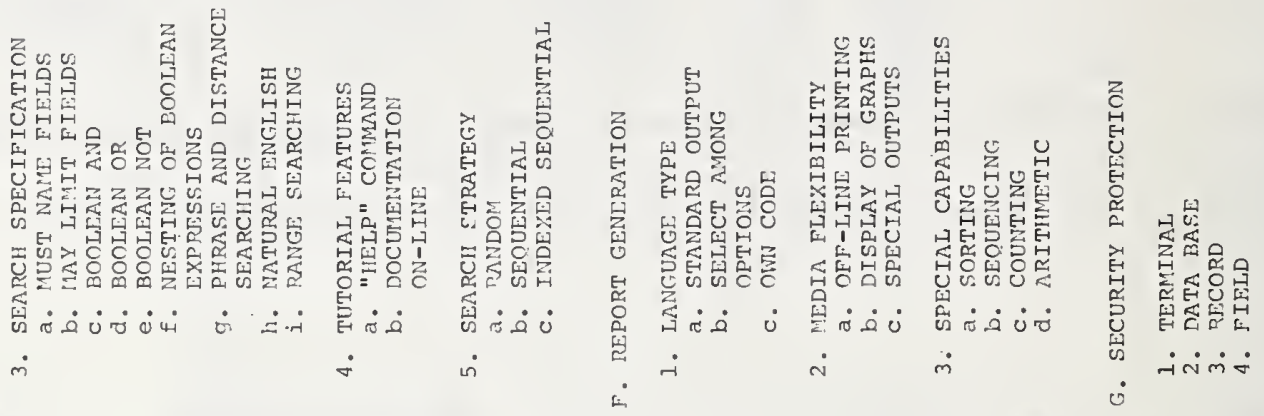

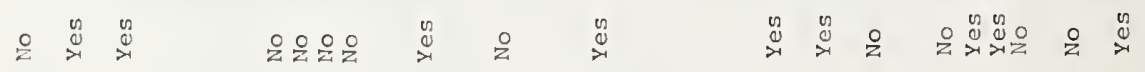
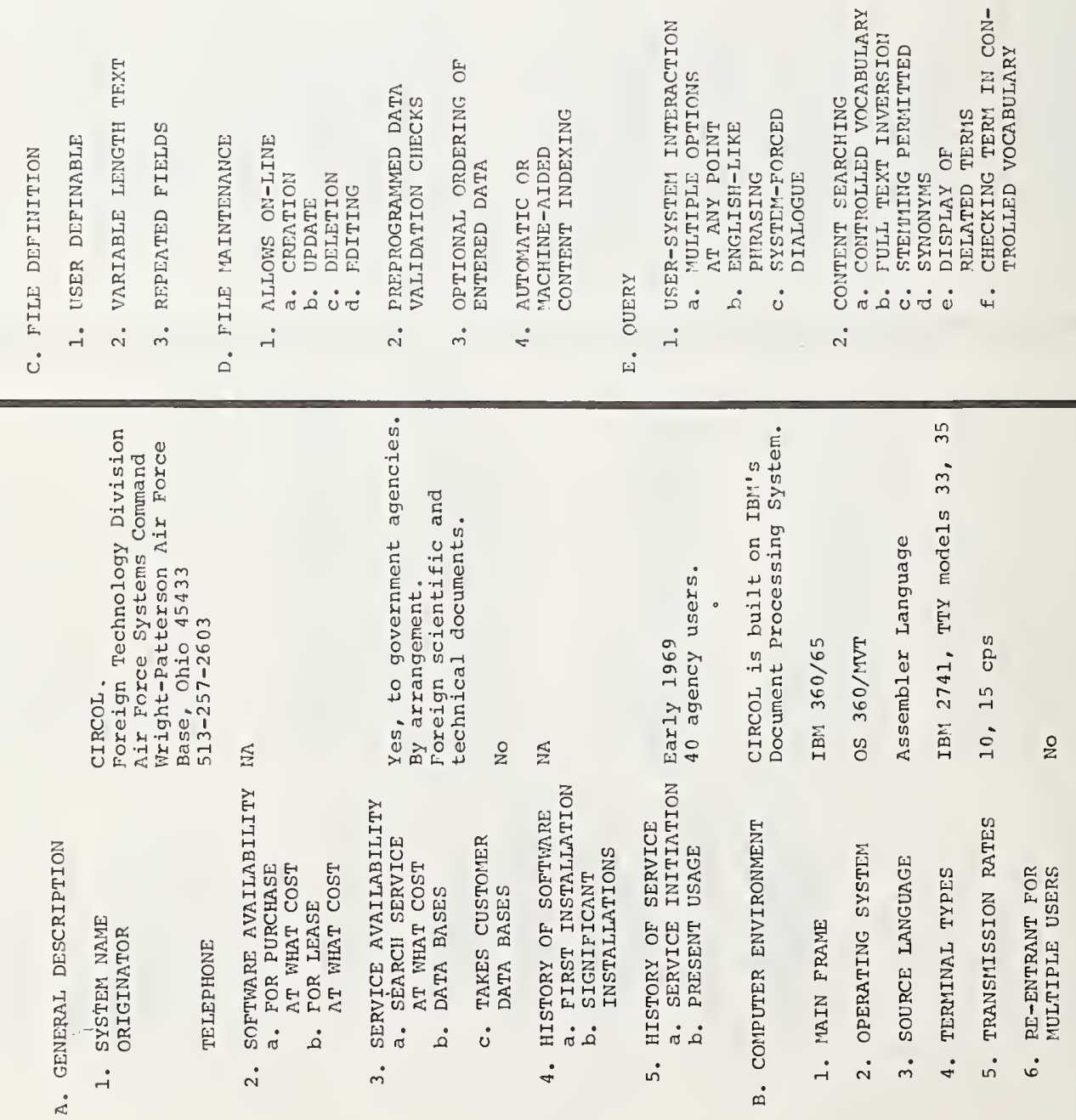


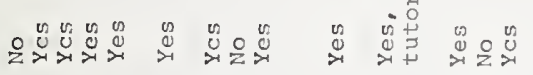

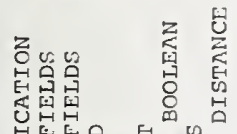

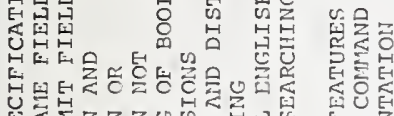

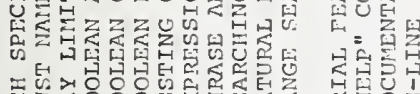

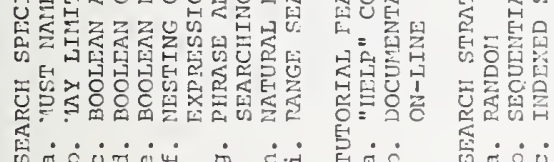

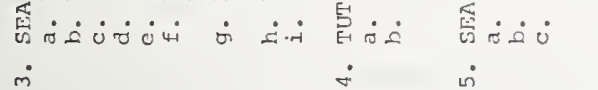

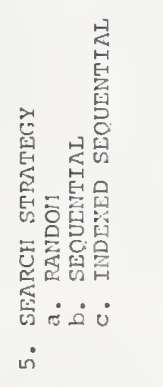

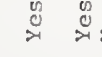

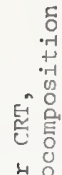

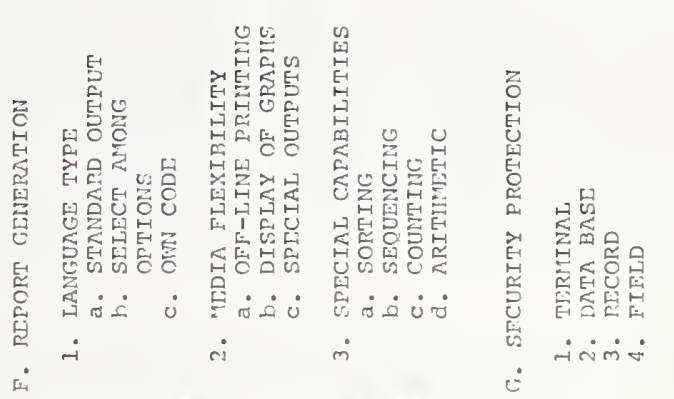

总

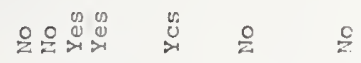

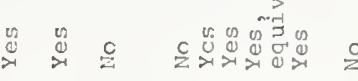

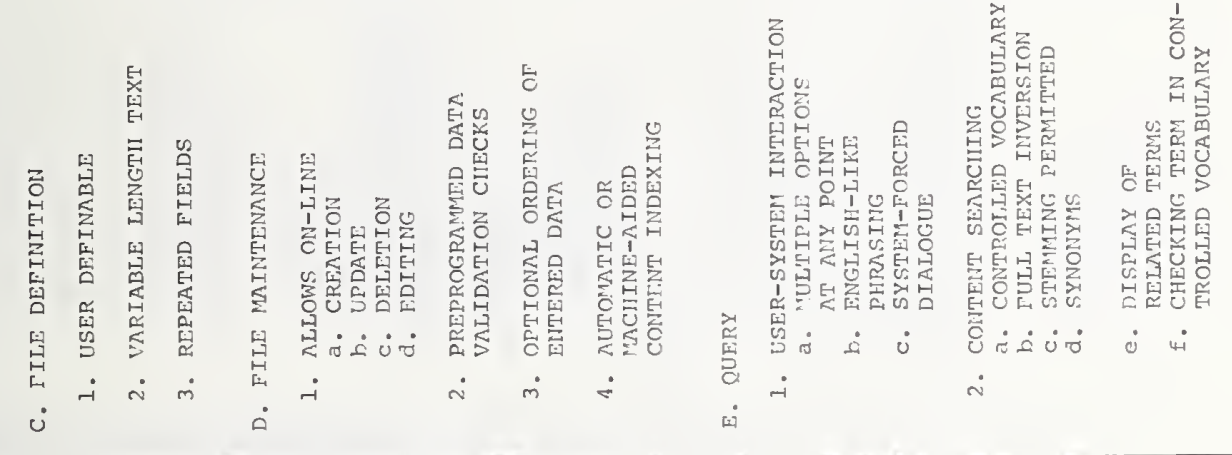

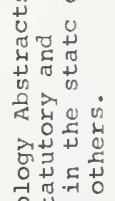

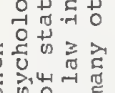

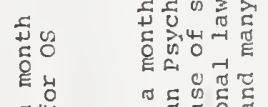

ता

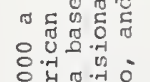

तิ

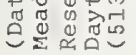

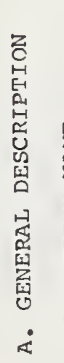

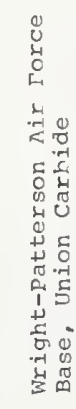

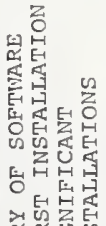

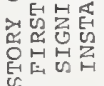
政

$$
\text { × }
$$

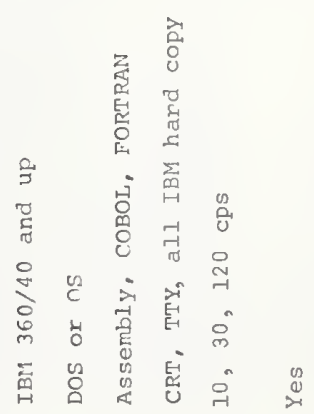




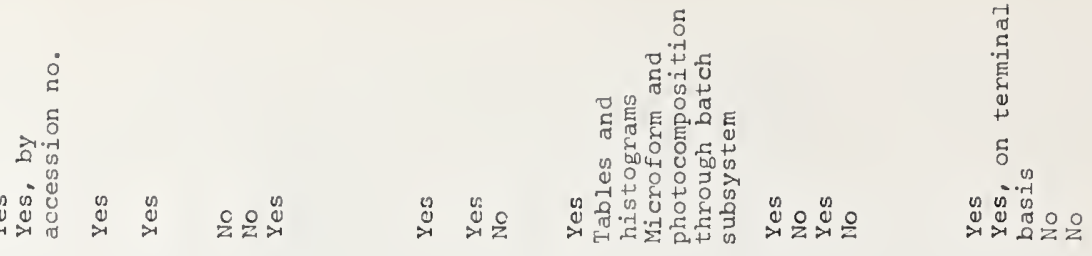

\section{咆鹃}

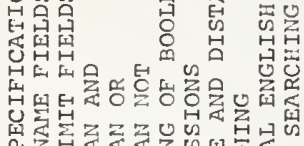

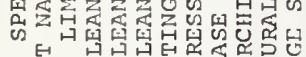

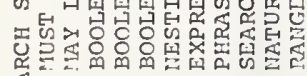

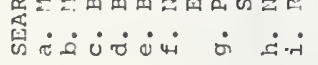
m
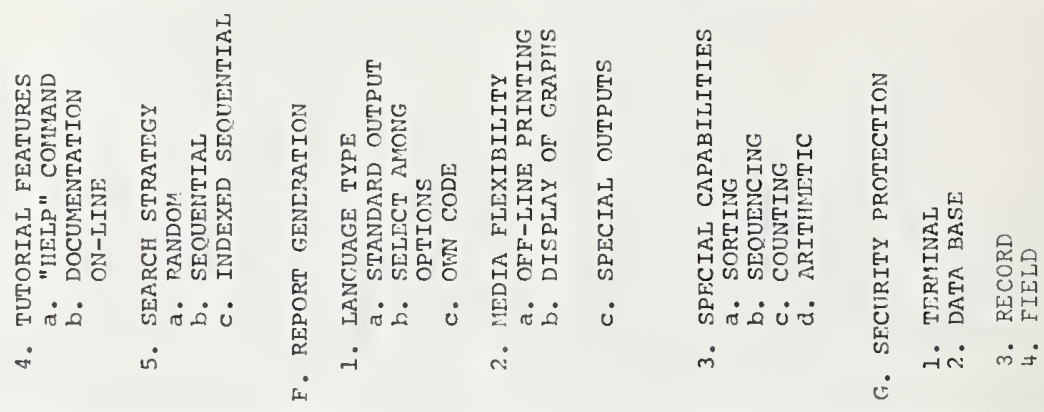

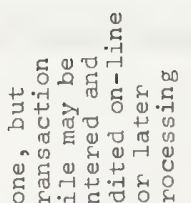

$\circ \stackrel{y}{z} \stackrel{y}{x}$

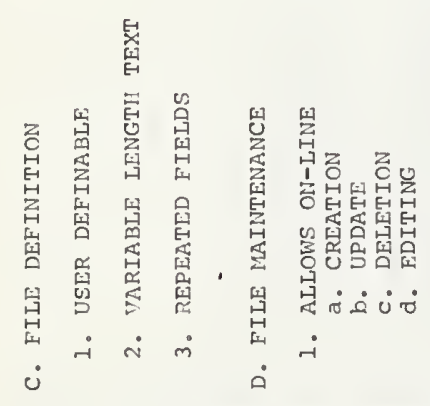

$\stackrel{\square}{\dddot{~}} \cong$

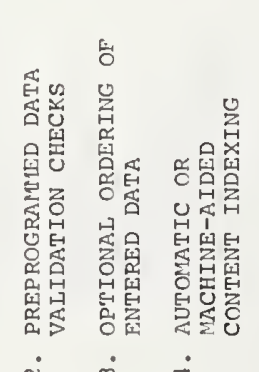

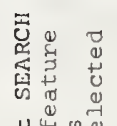

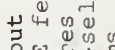

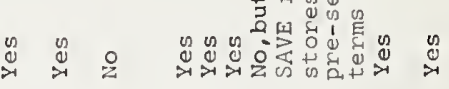

zo

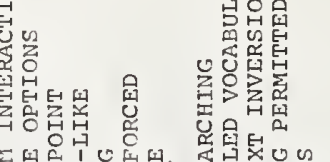

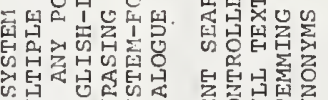

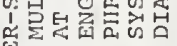

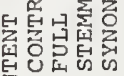

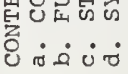

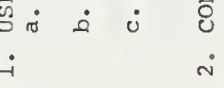

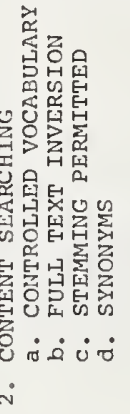

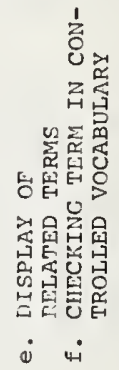

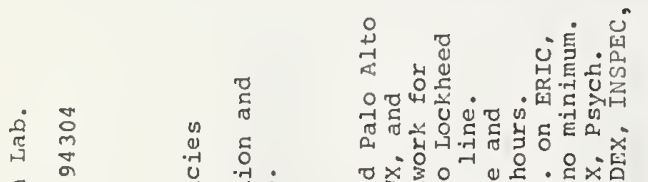

万人

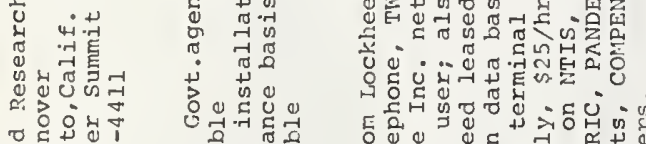

ช.

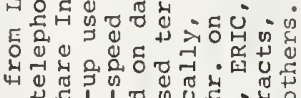

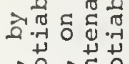

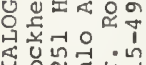

-

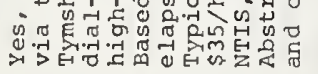

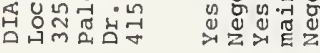

Z

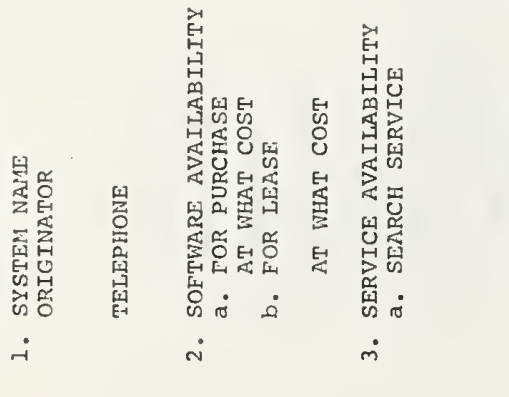

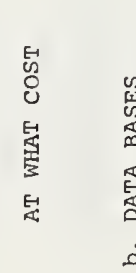
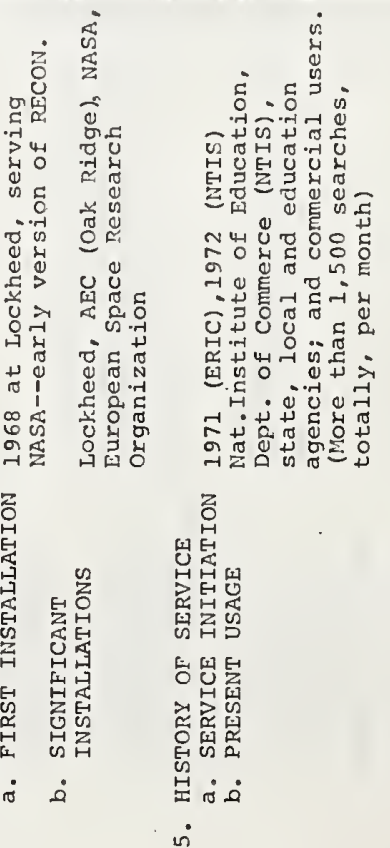

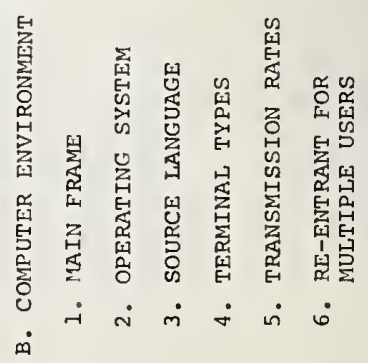




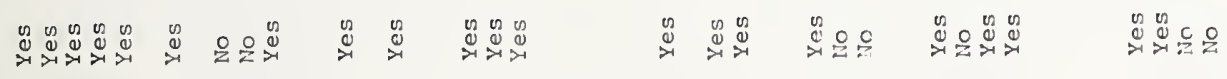
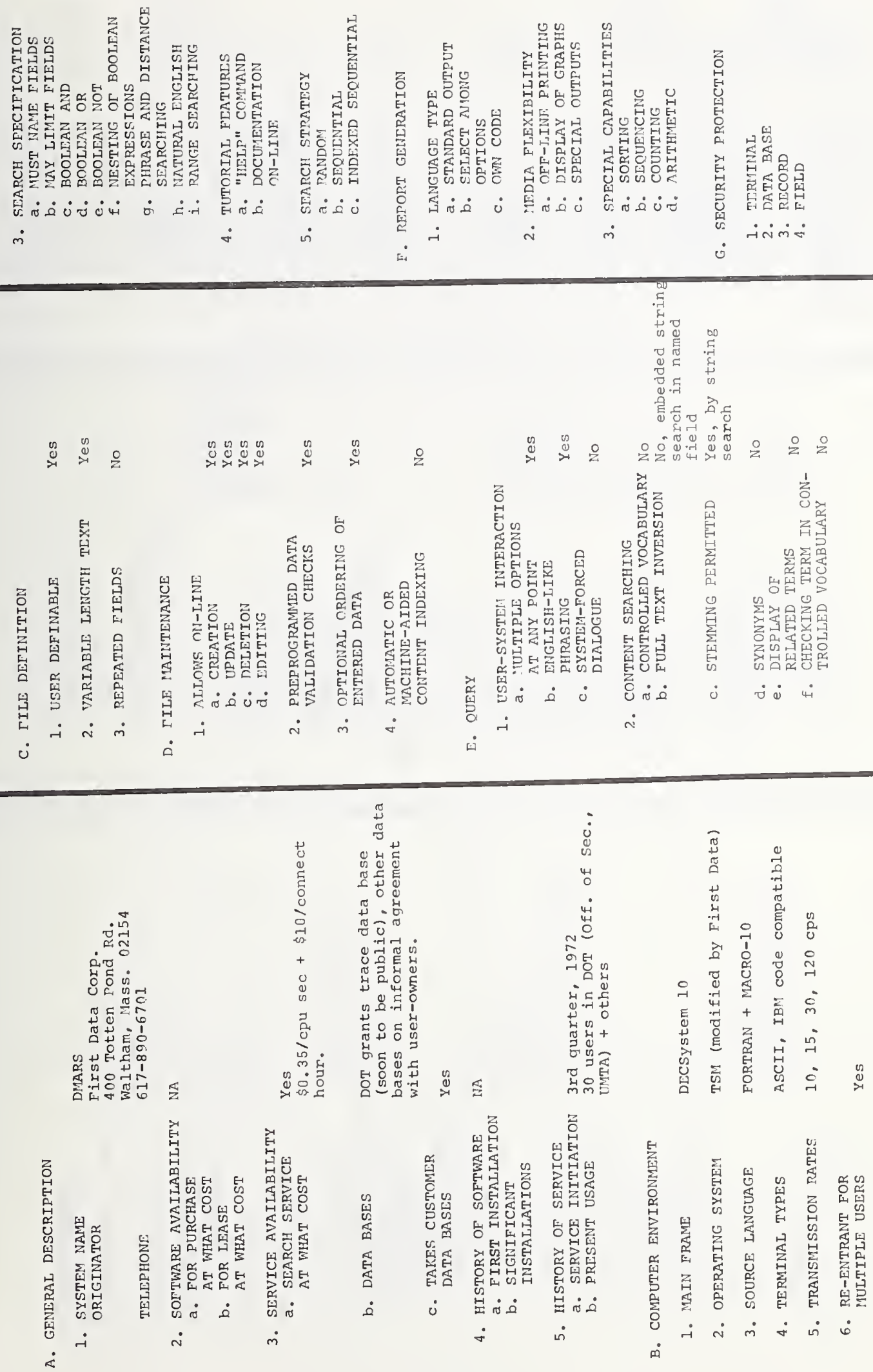


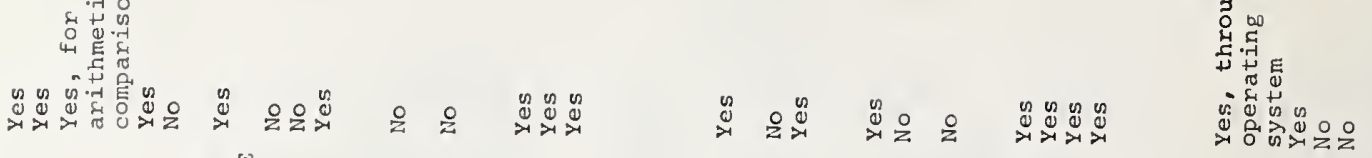

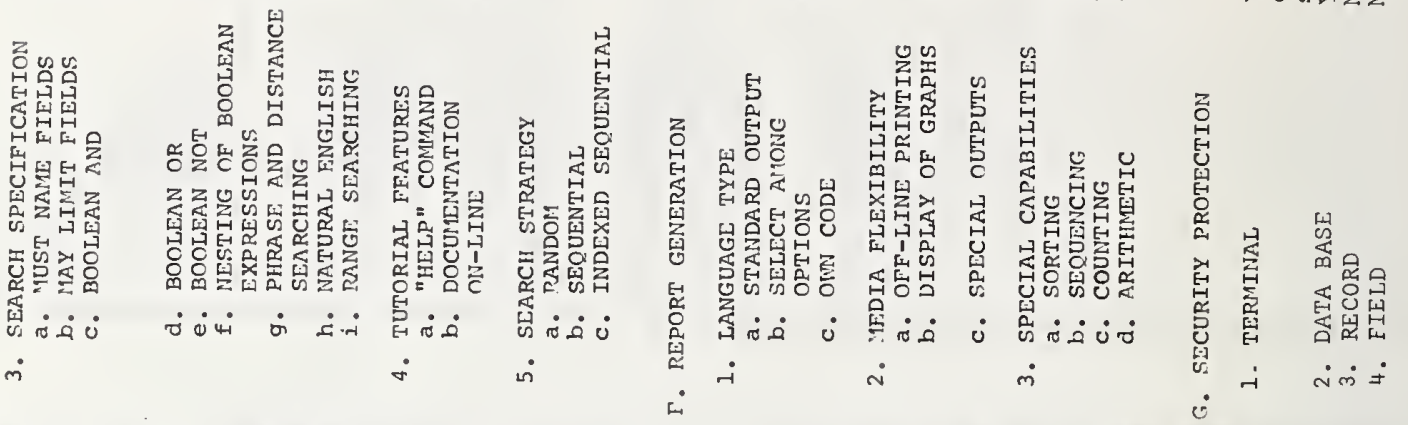

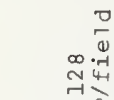

g)
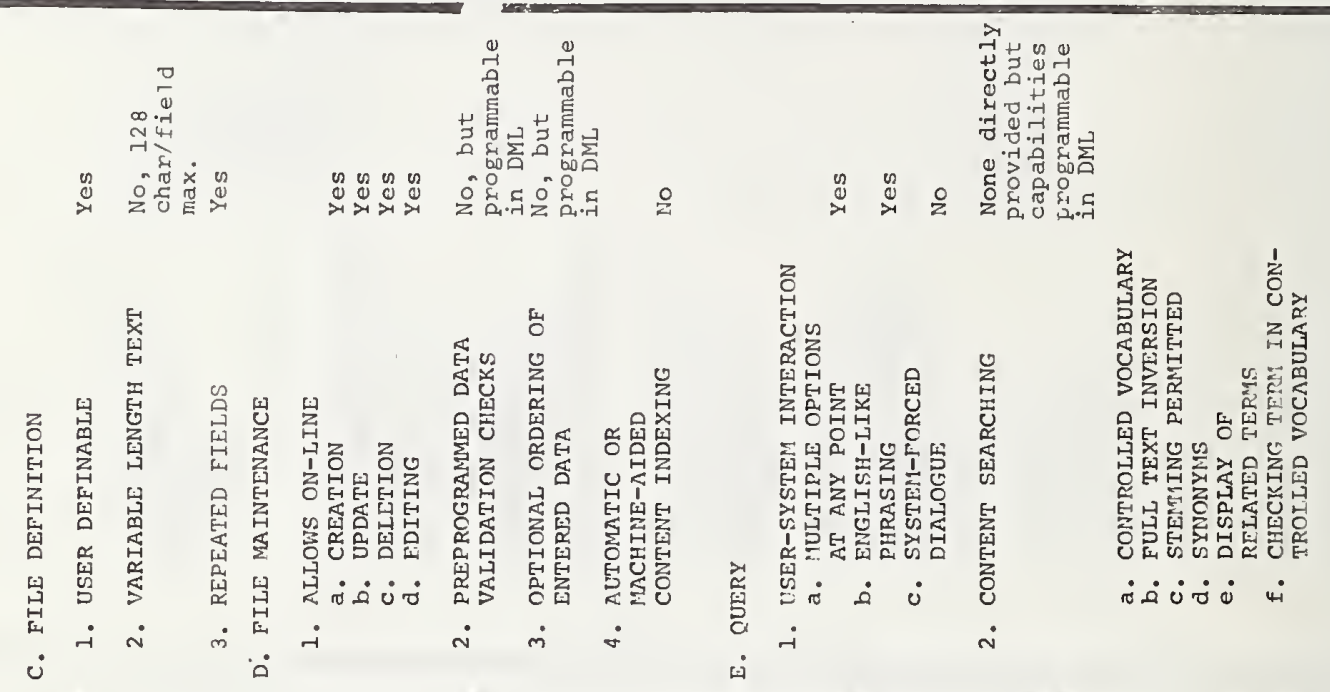

ธี

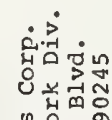

造

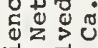

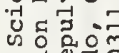

मํํำ

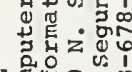

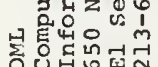

ฮ

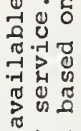

in

空学

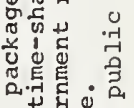

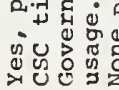
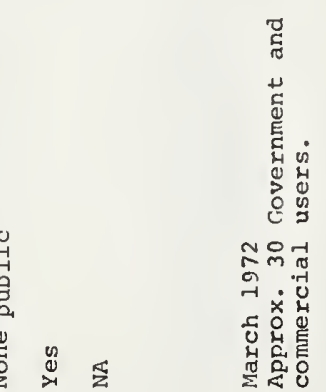

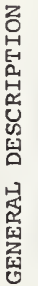

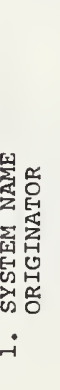

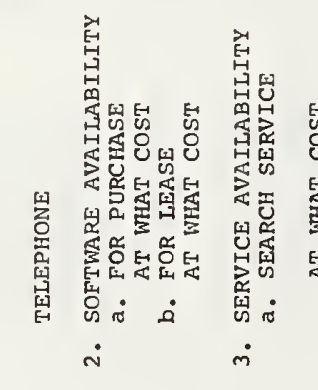

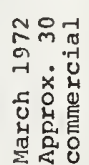

啚

$\ddot{m}$

लिं

势

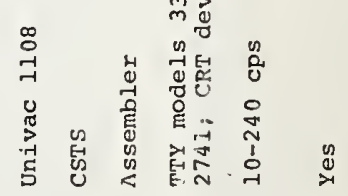

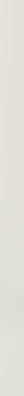




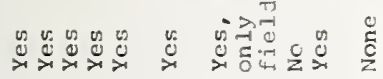

\section{올 룼돌}

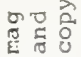

㺟

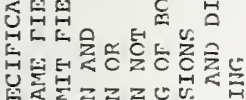

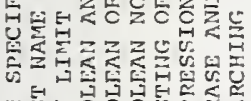

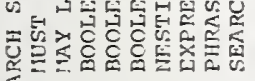

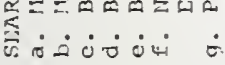
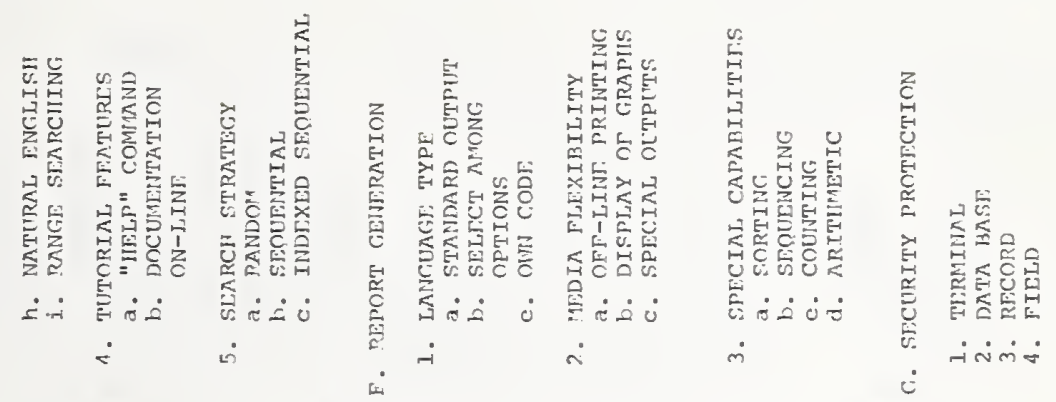

要

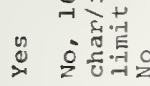

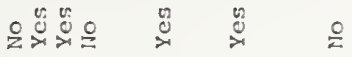

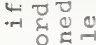

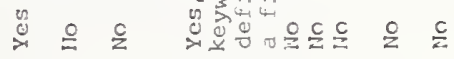

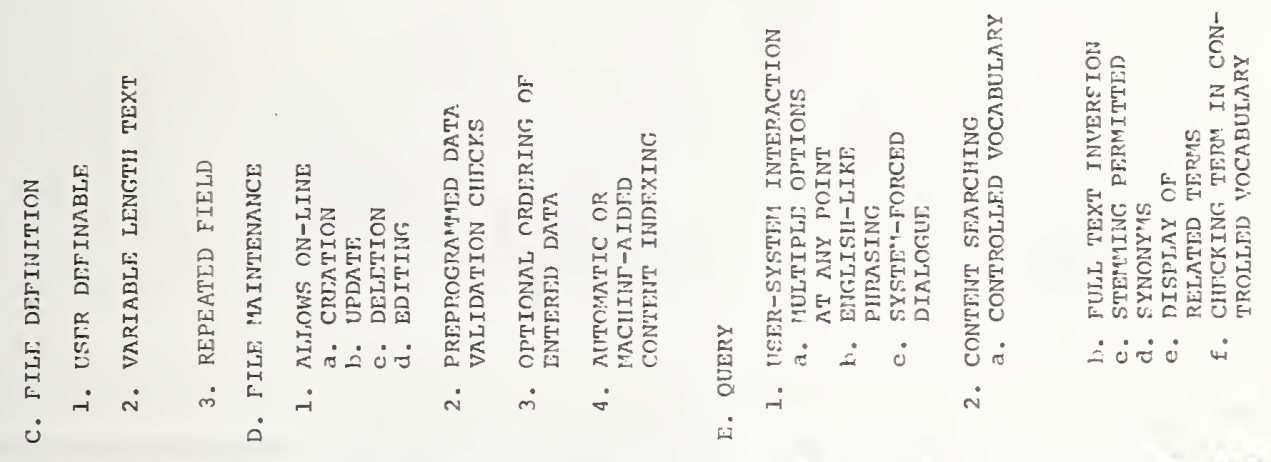

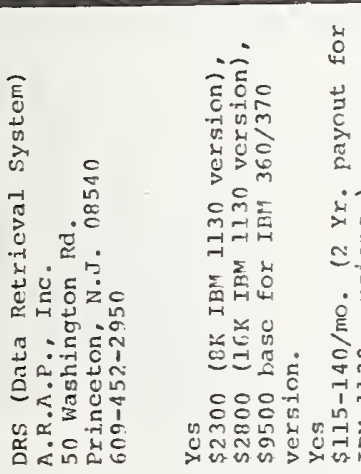

音

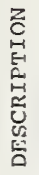

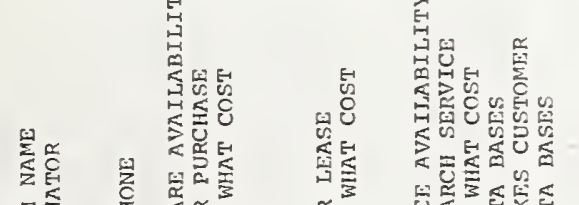

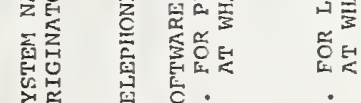

尡总

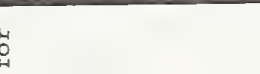

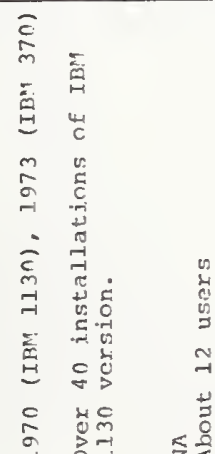

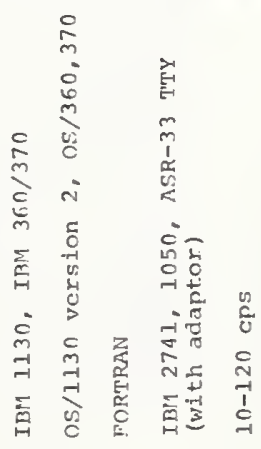

웅

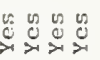




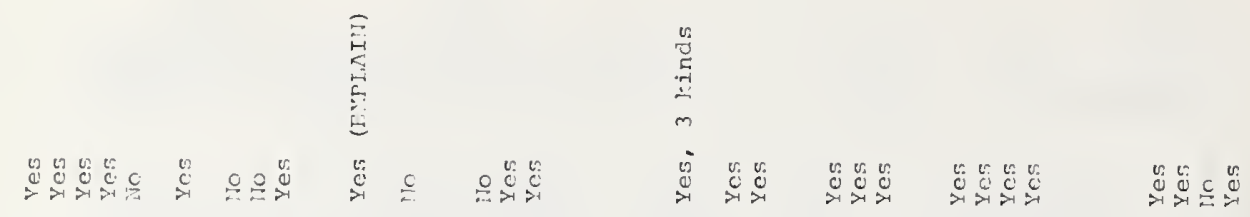

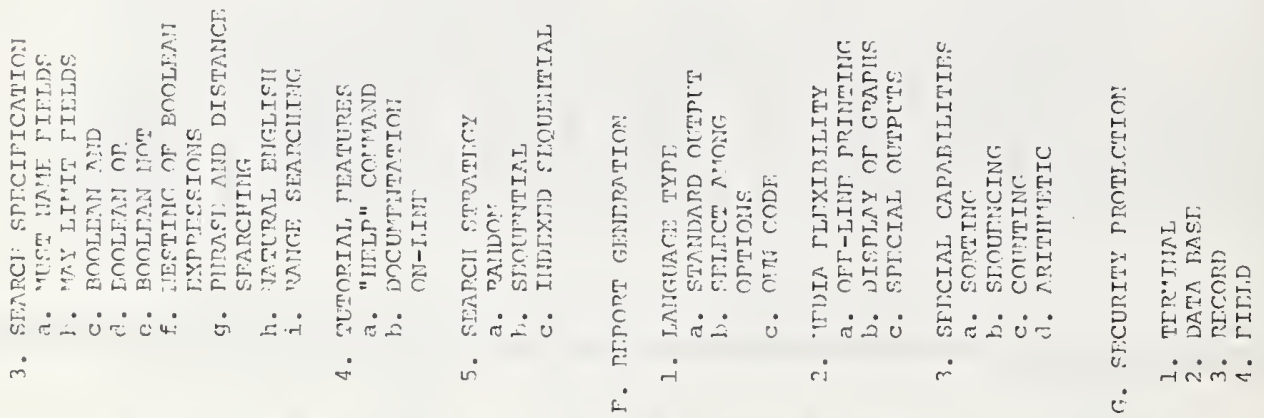

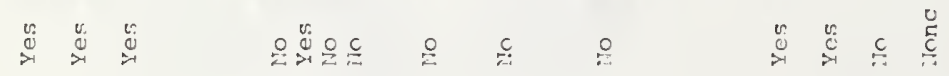

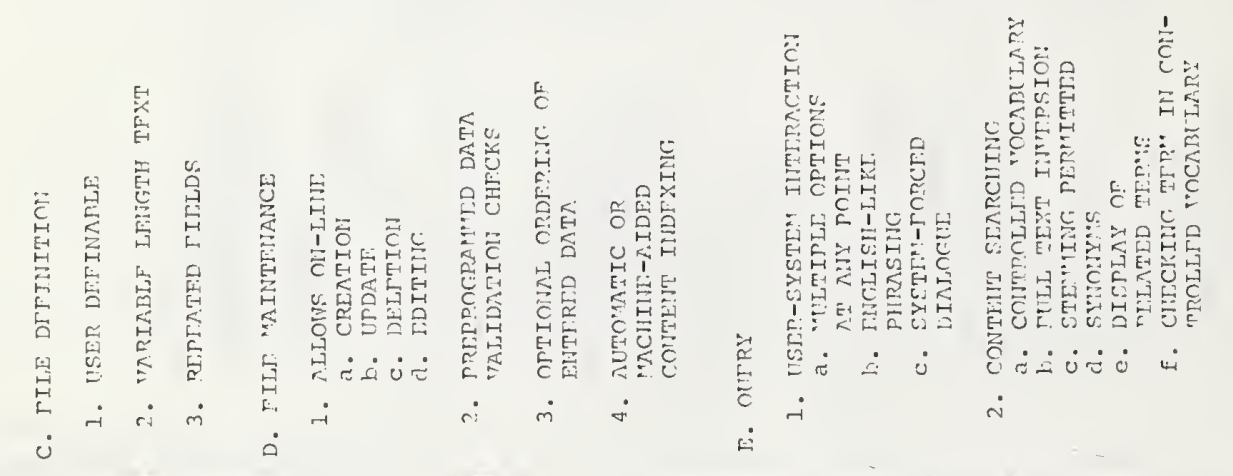

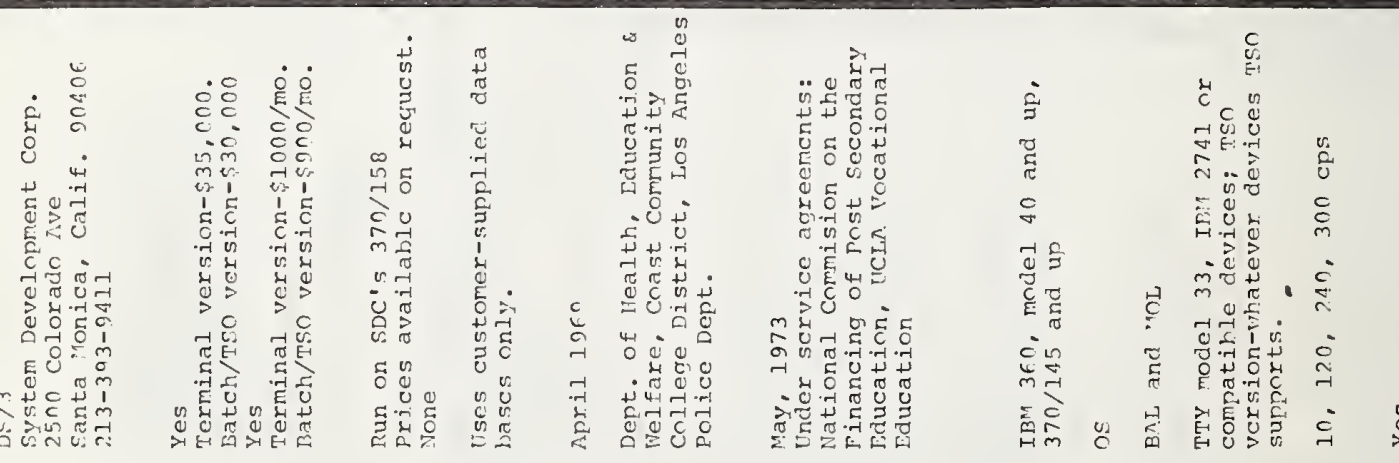

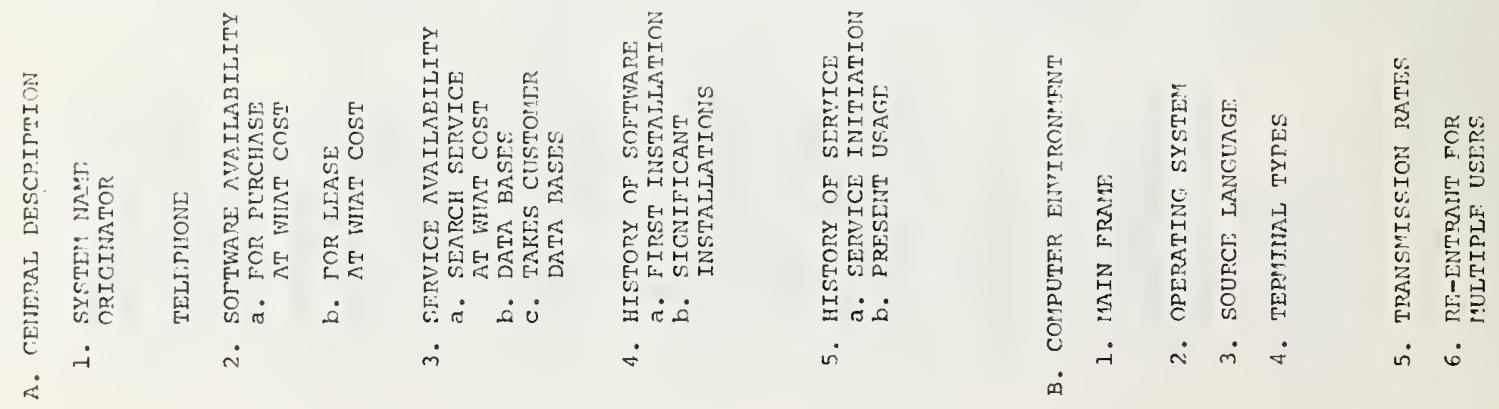





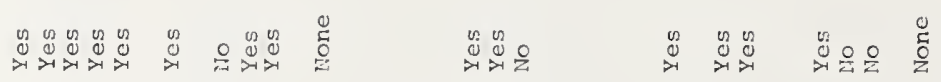

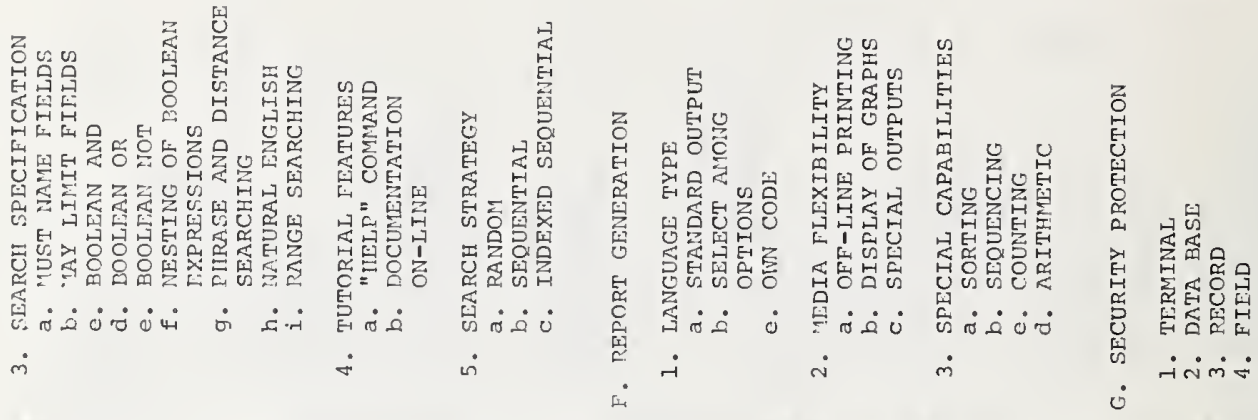

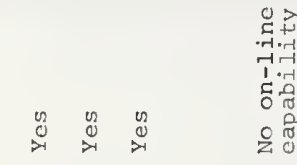

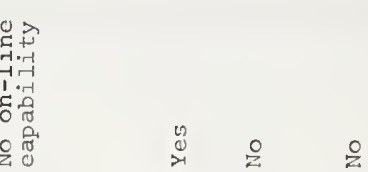

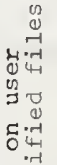
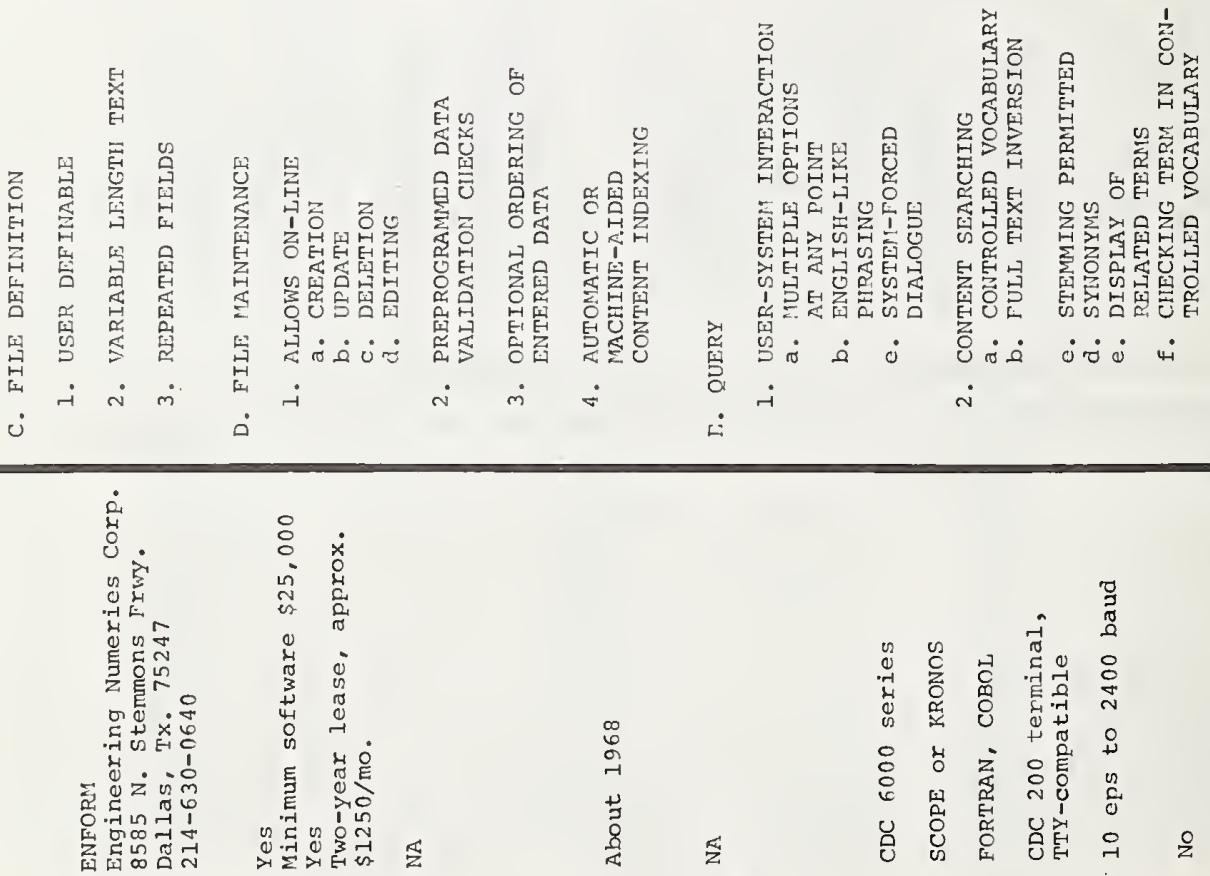

文

节

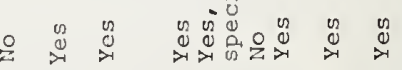

要

要

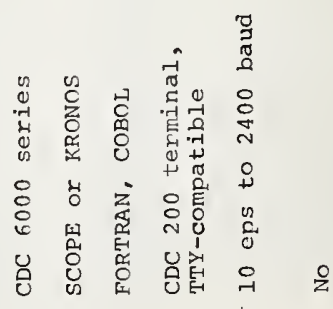

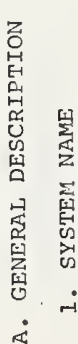
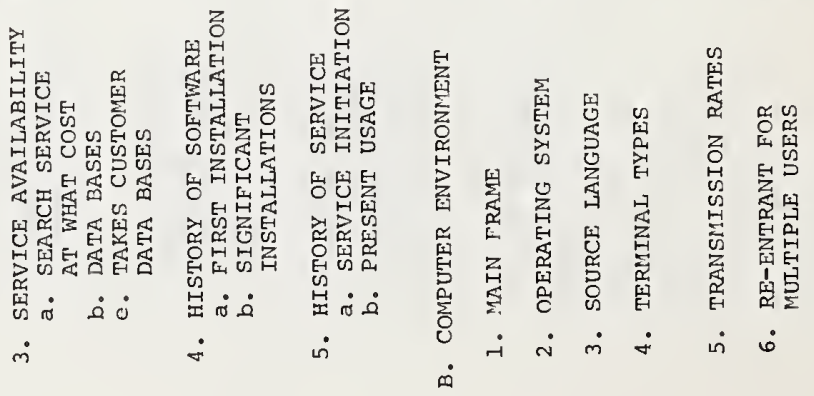


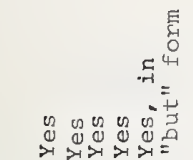

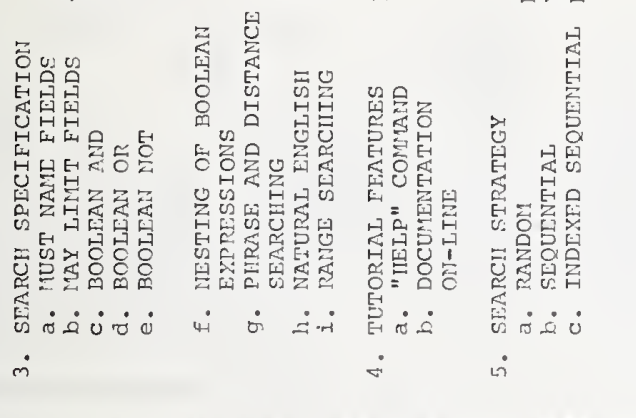

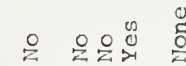

$c \stackrel{0}{0} 0$

$\stackrel{0}{2}$

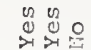

$\sum_{x \rightarrow 0}^{0} \int_{\substack{0 \\ 0}}^{0}$

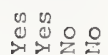

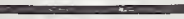

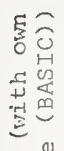

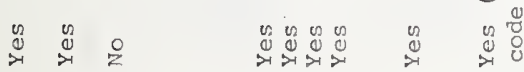
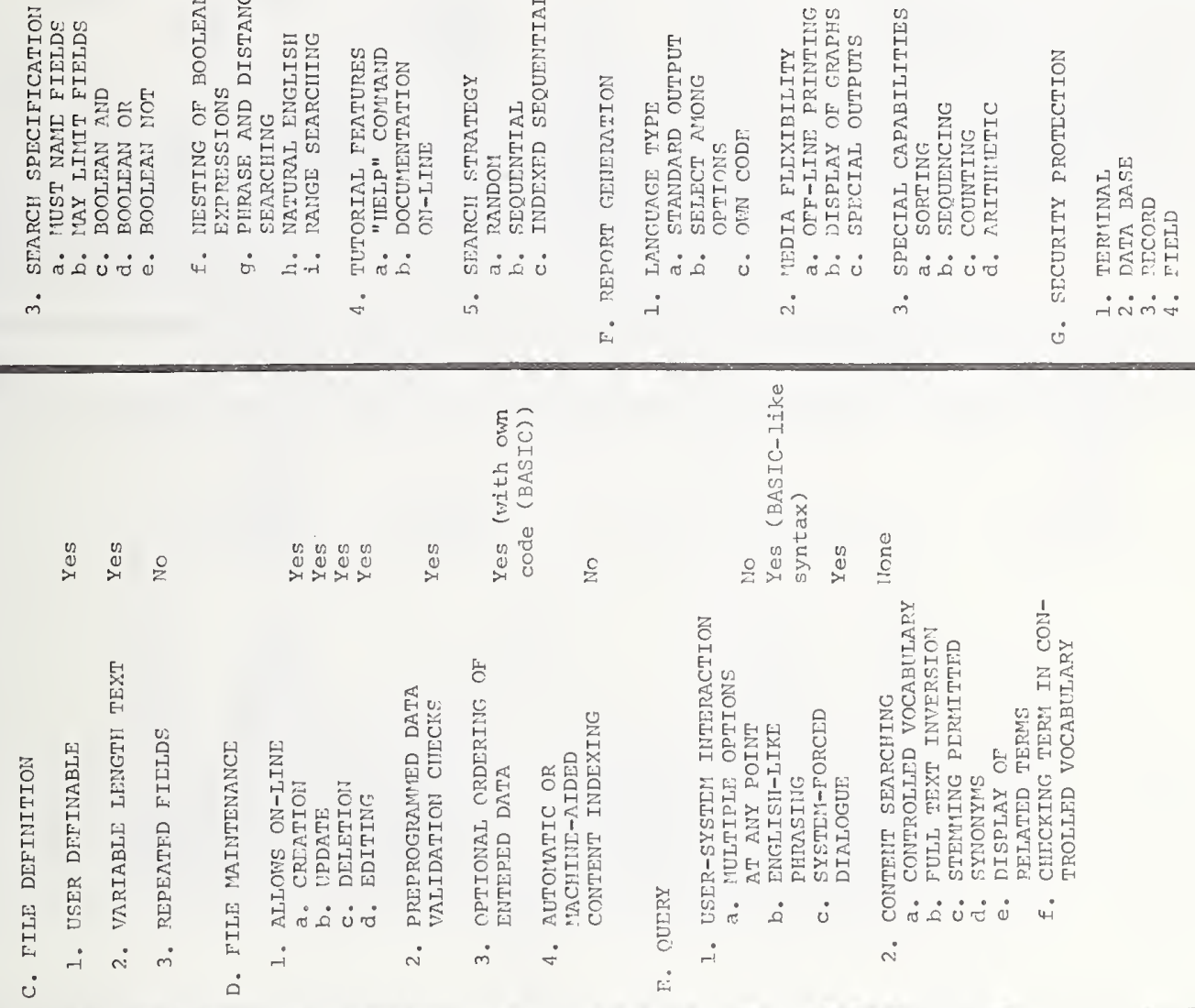

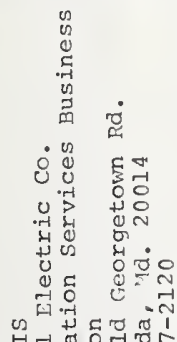

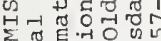

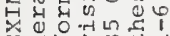

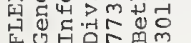

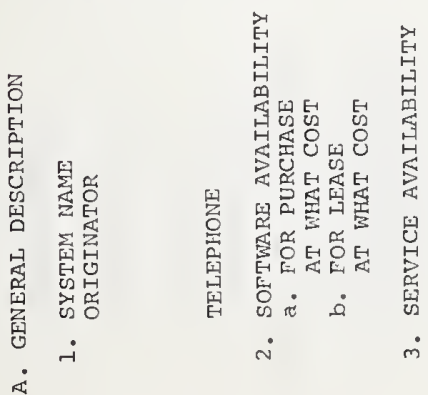

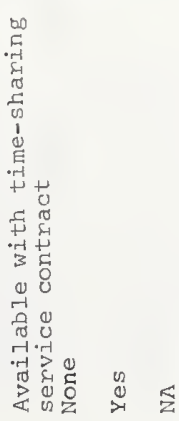
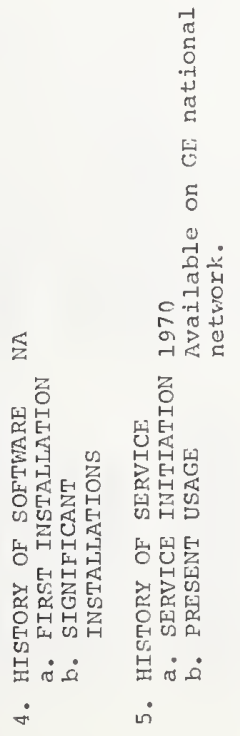

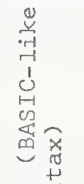

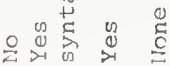

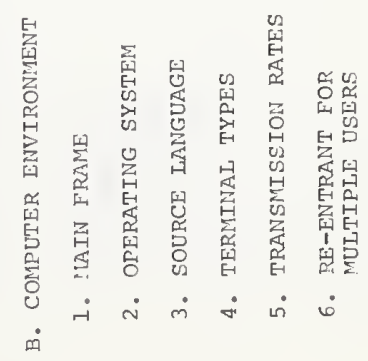




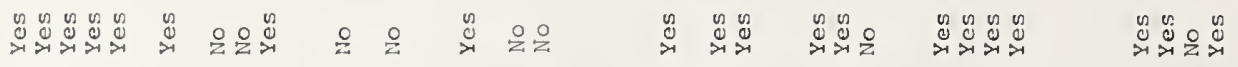

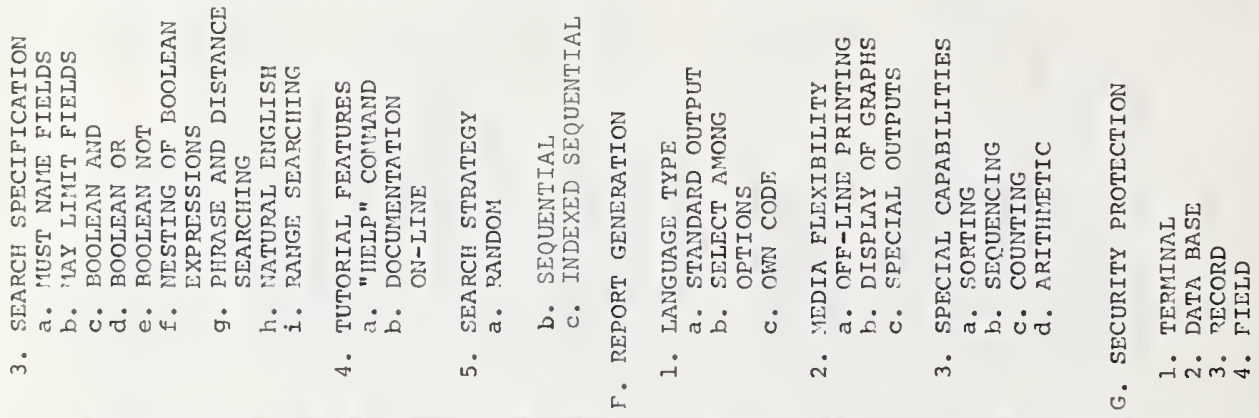

品高

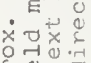

है

吕出的.

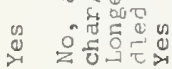

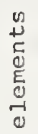

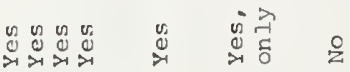

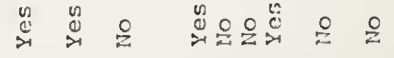

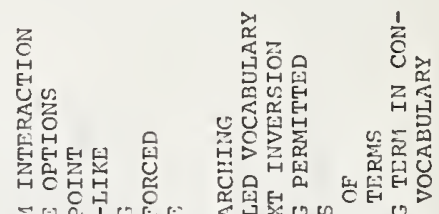

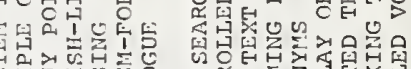

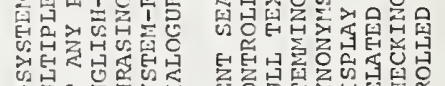

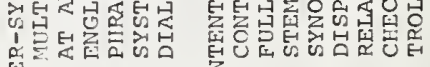

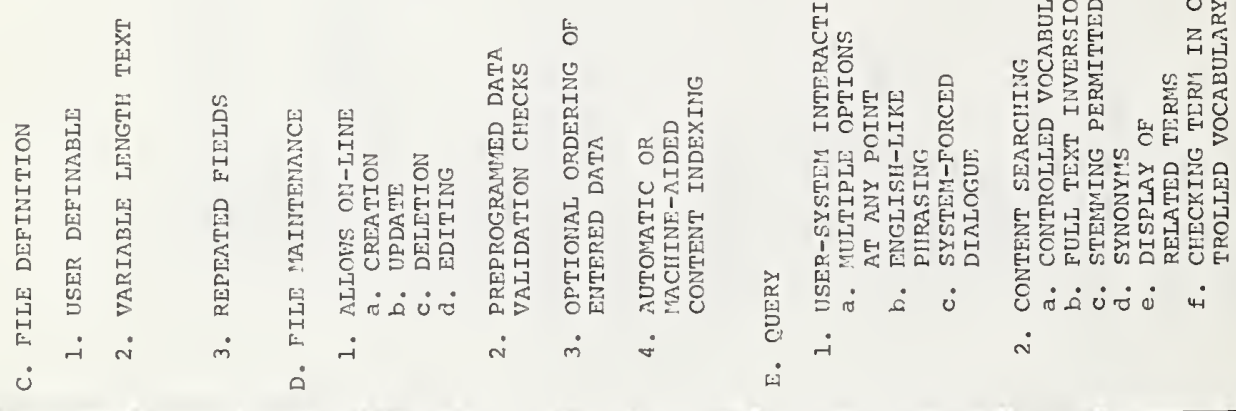

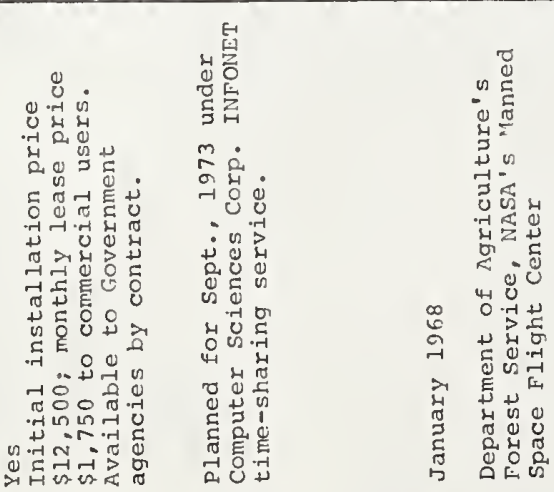

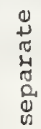

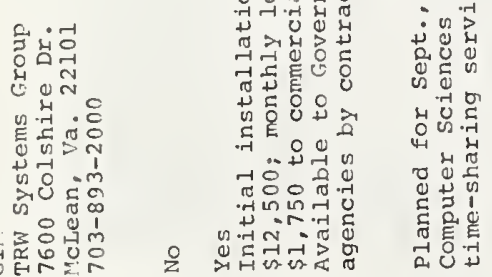

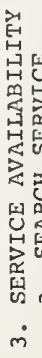

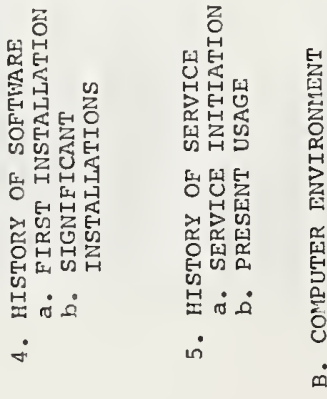

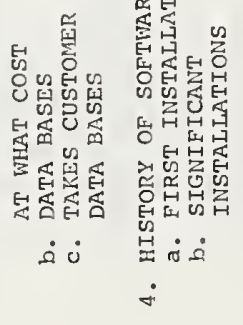

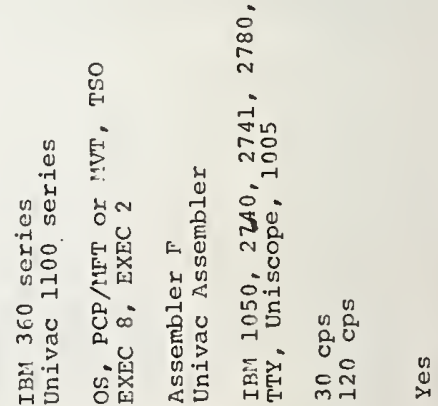

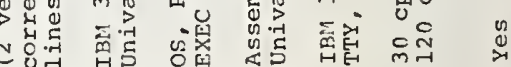
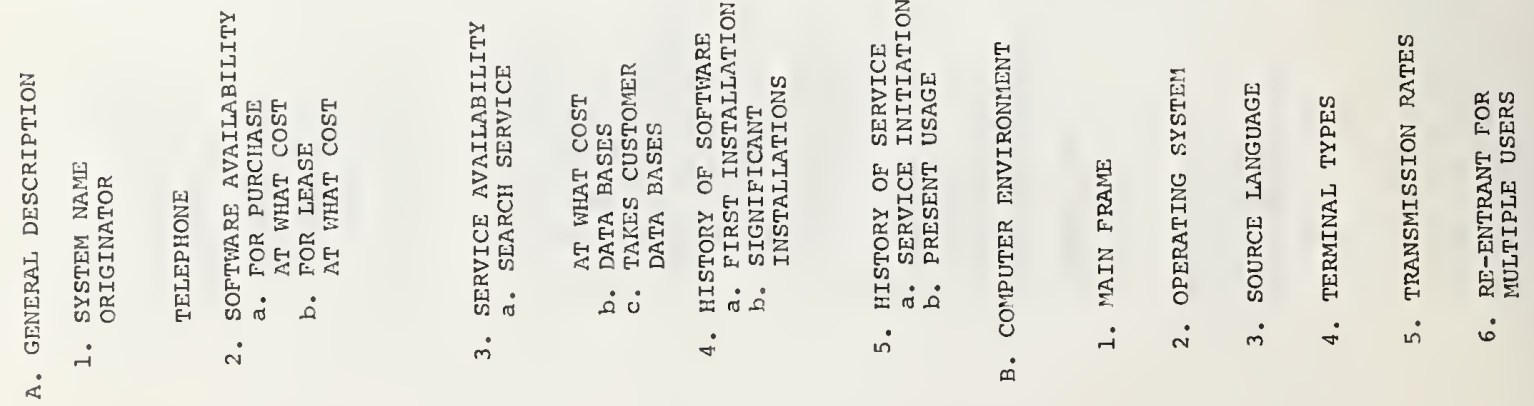


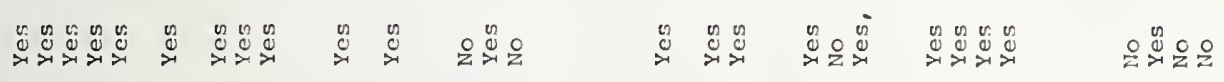
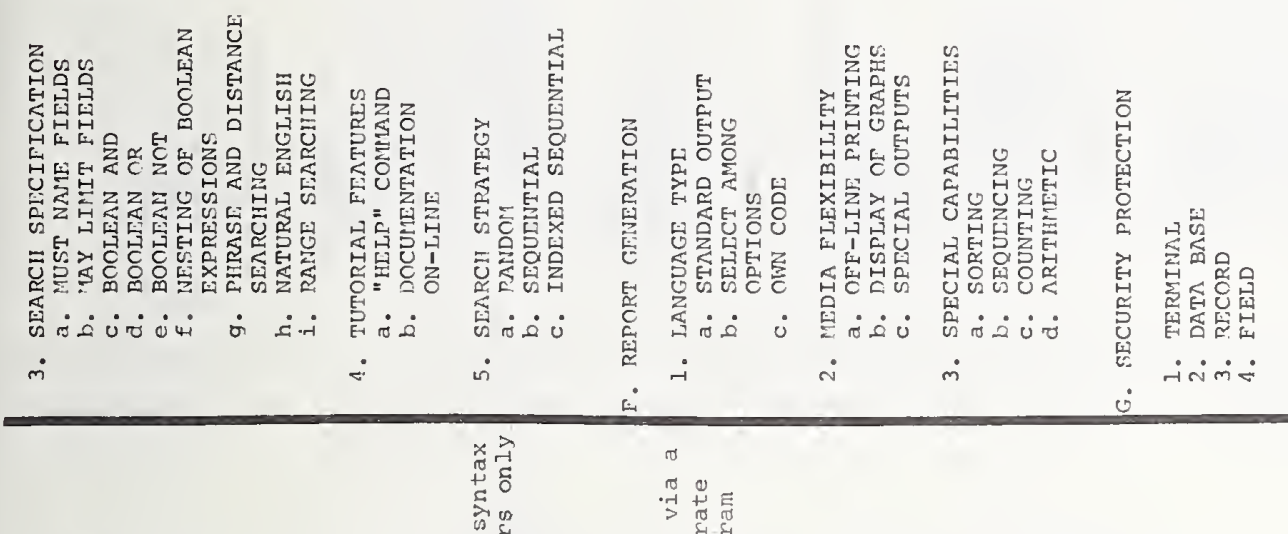

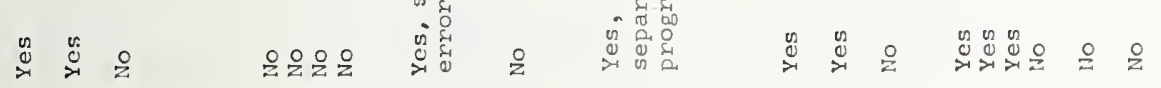
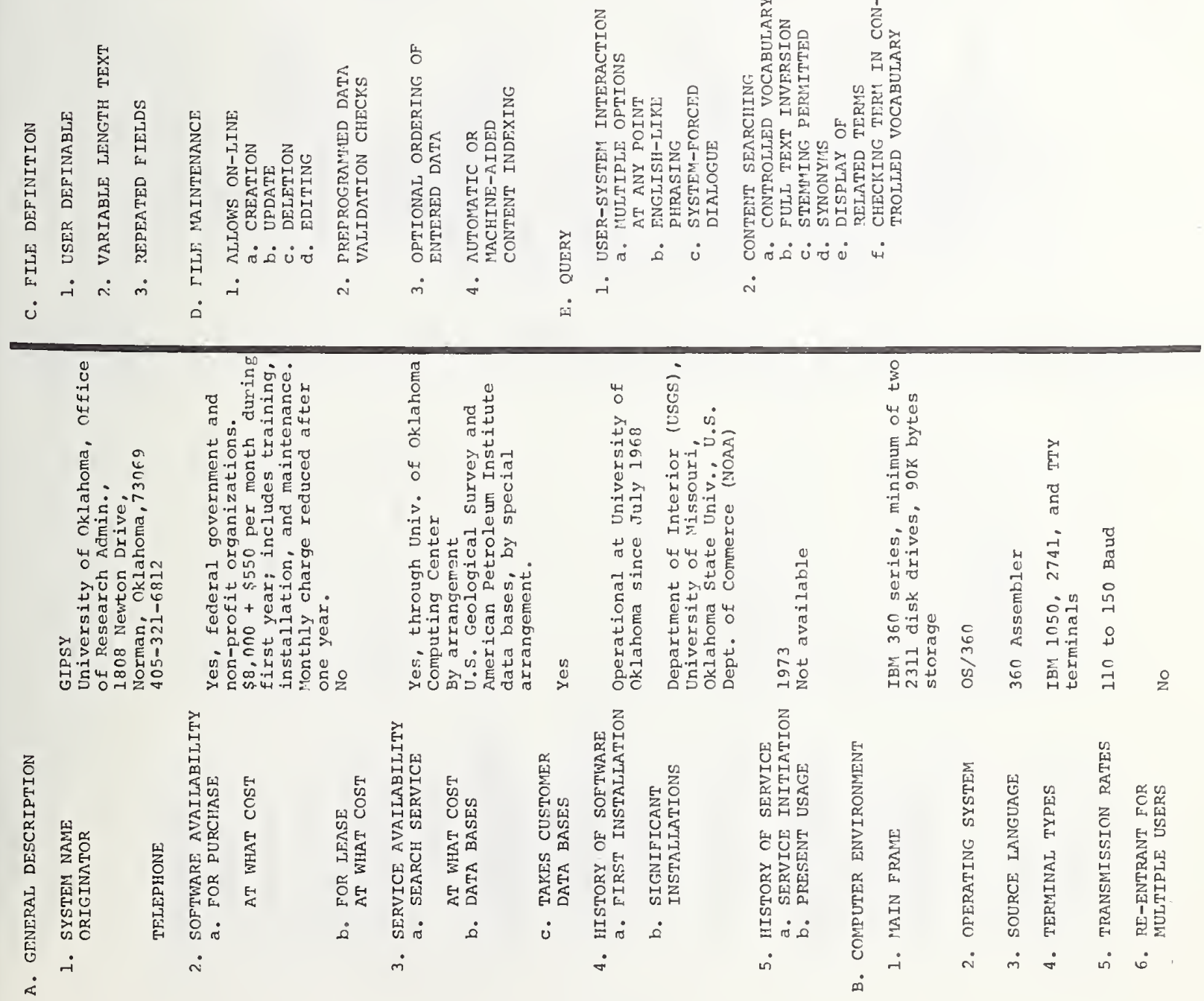


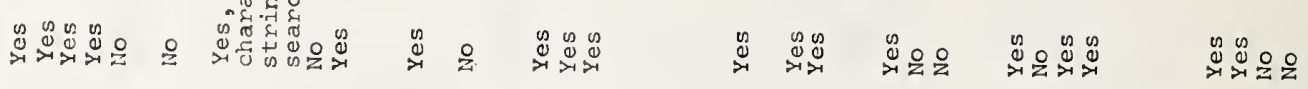

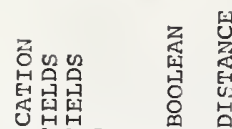

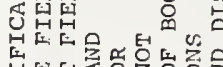

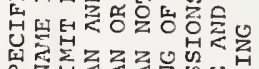

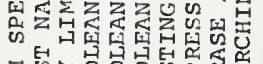

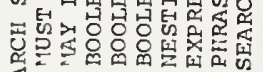

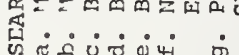

$$
\dot{m}
$$

$\dot{m}$

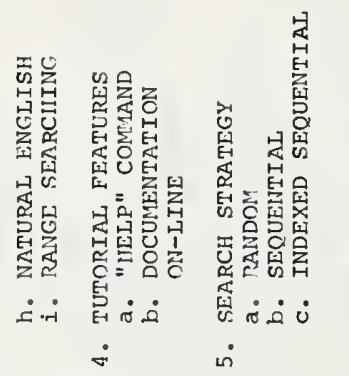

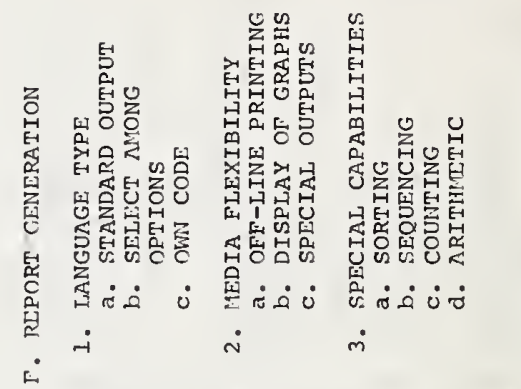

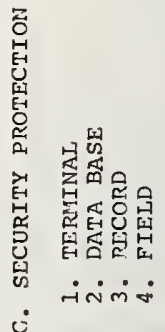

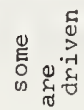

$\begin{array}{ccc}-1 & 0 \\ \pi & 0 \\ -\pi & 0 \\ -7 & 0\end{array}$

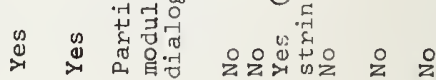

总

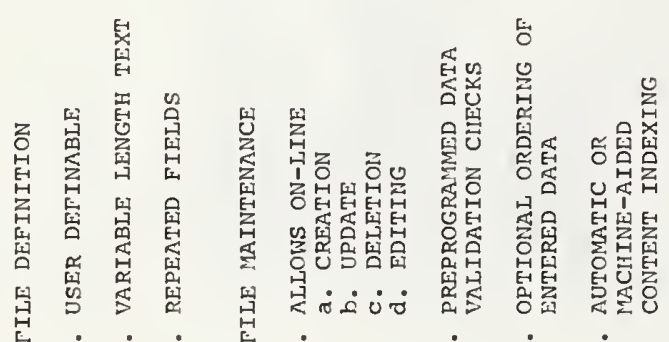

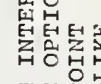

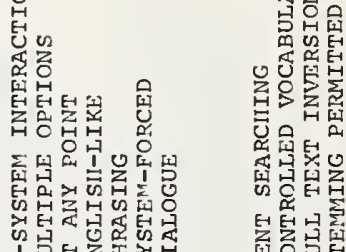

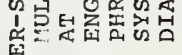

密 $\dot{0} \dot{0}$

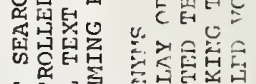

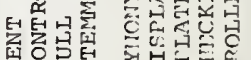

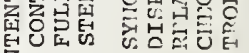

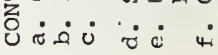
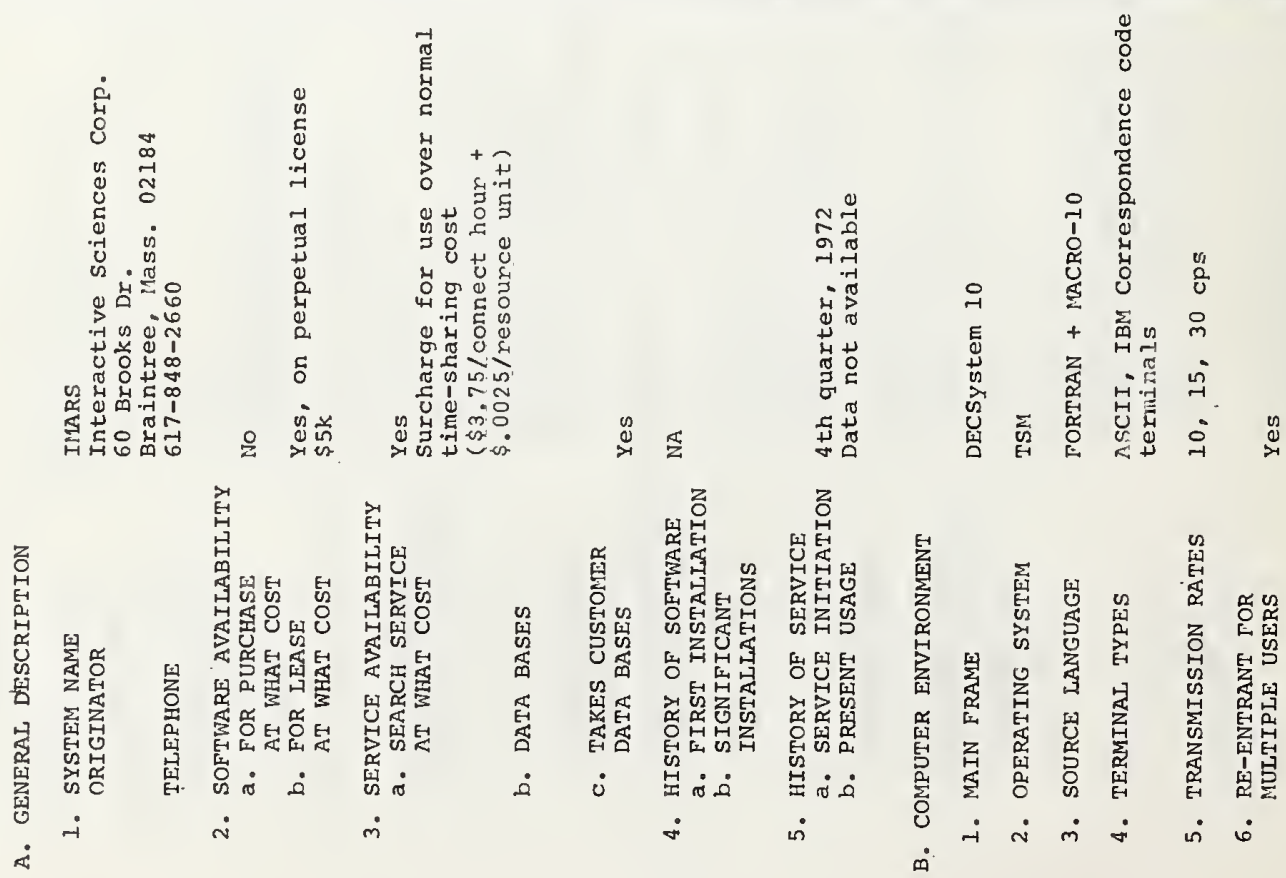

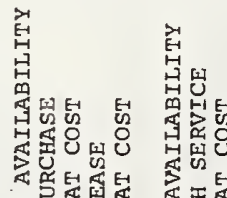

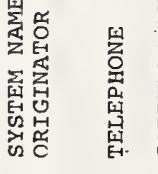

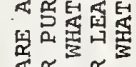

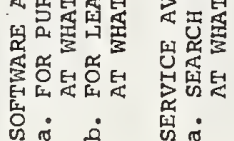
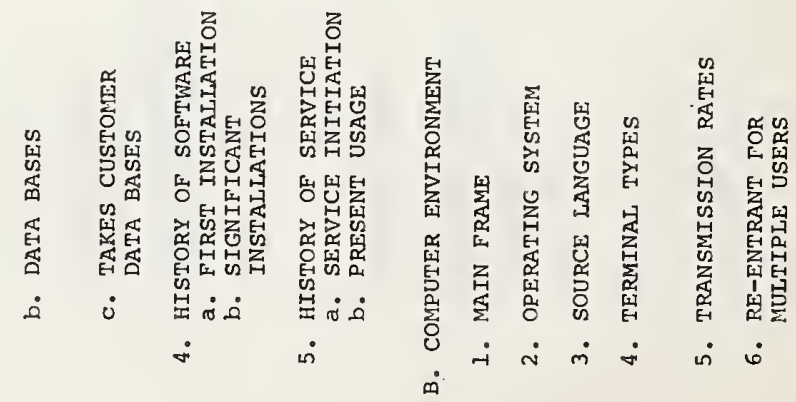


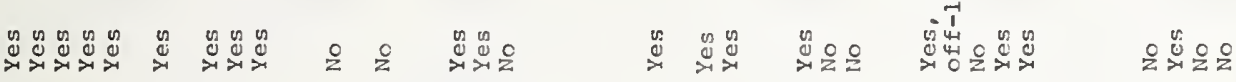

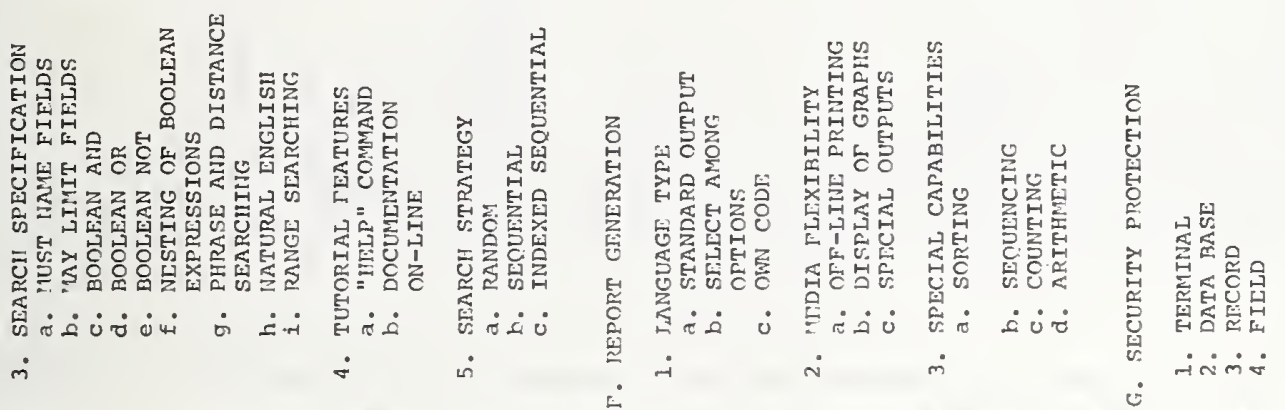

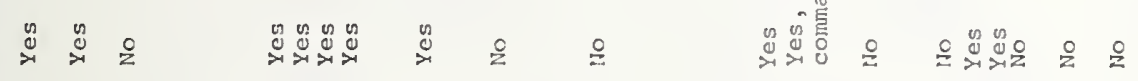
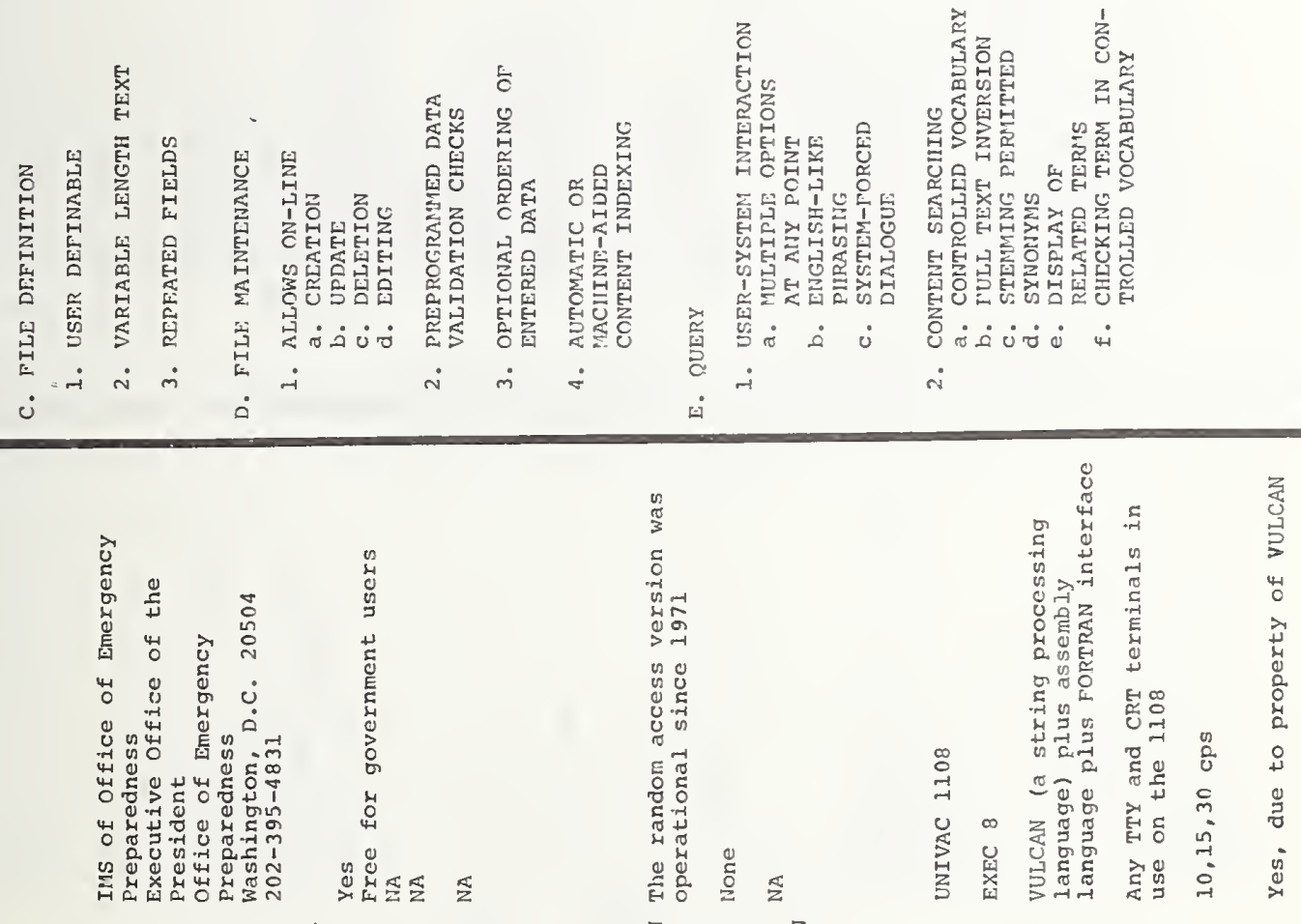


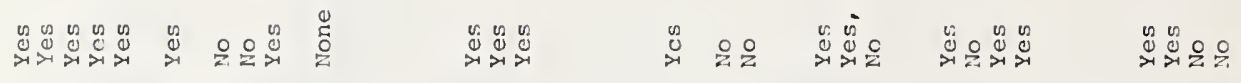

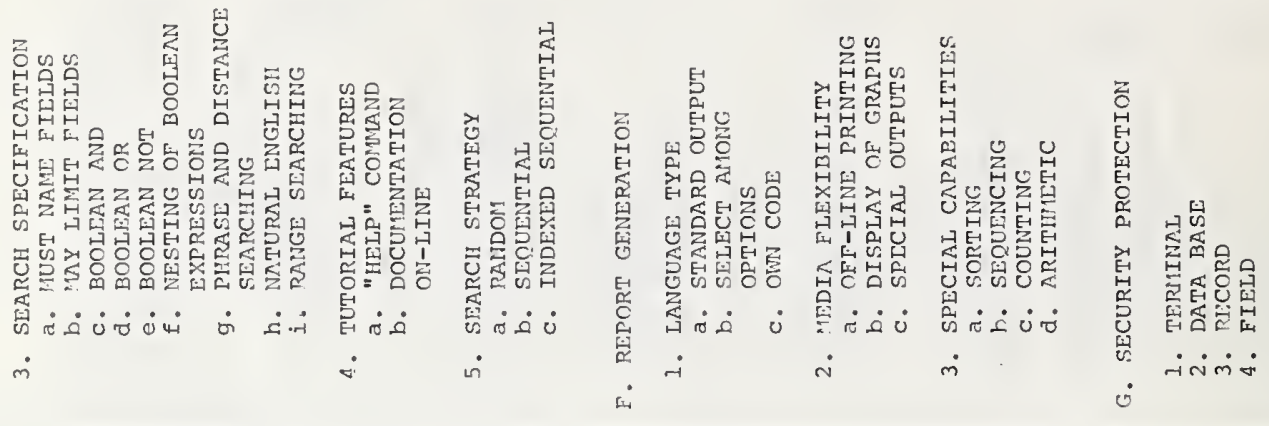

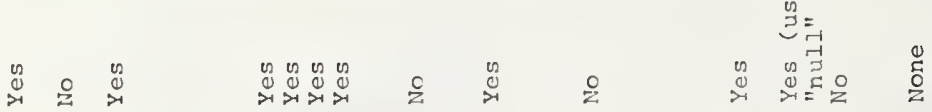
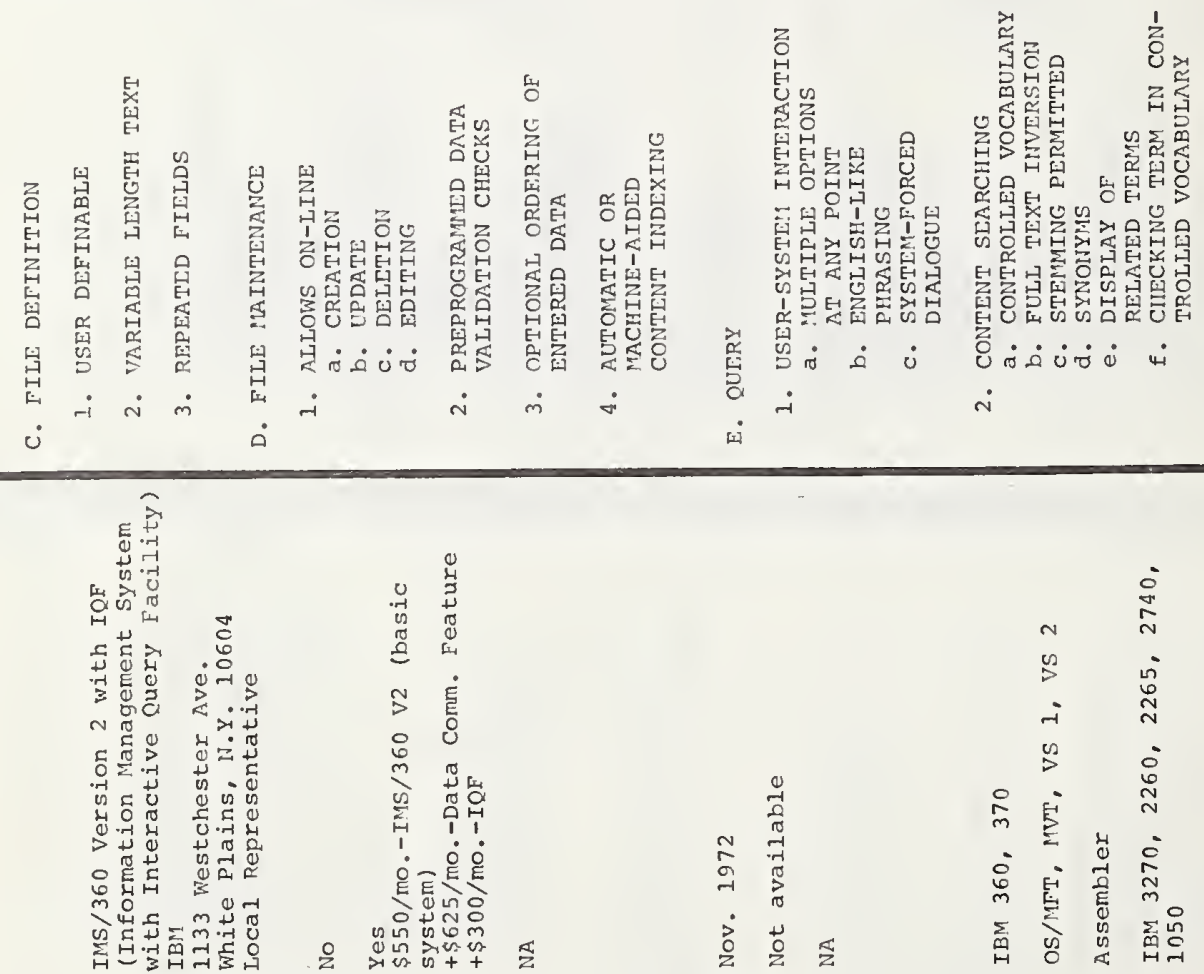

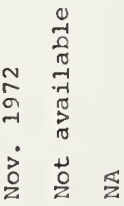

密

寻先昆 今

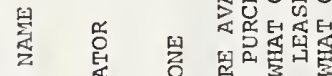

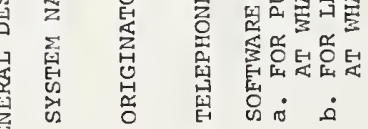

鬼

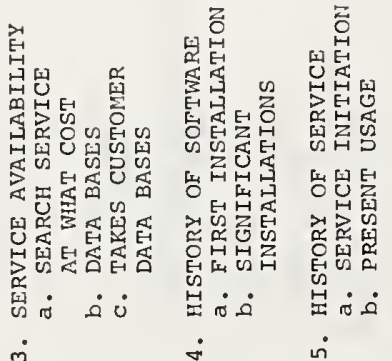

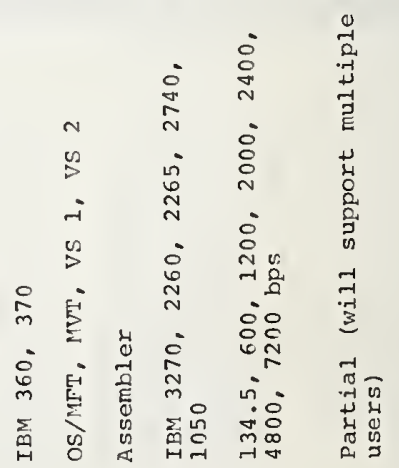

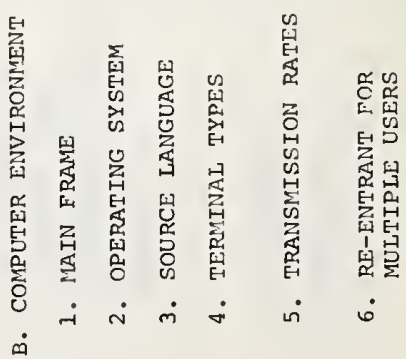




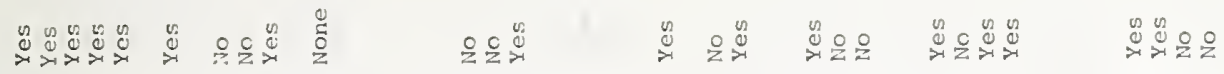

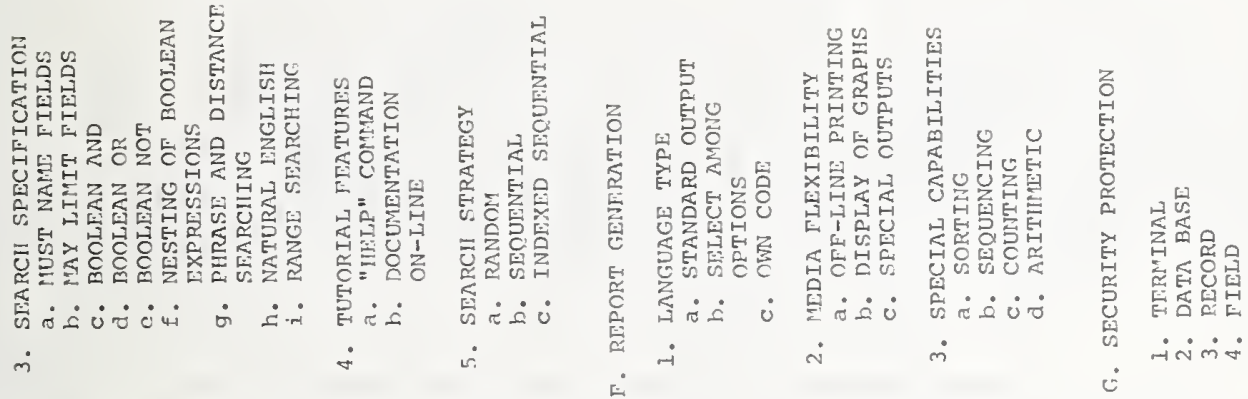

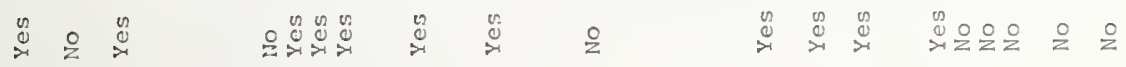
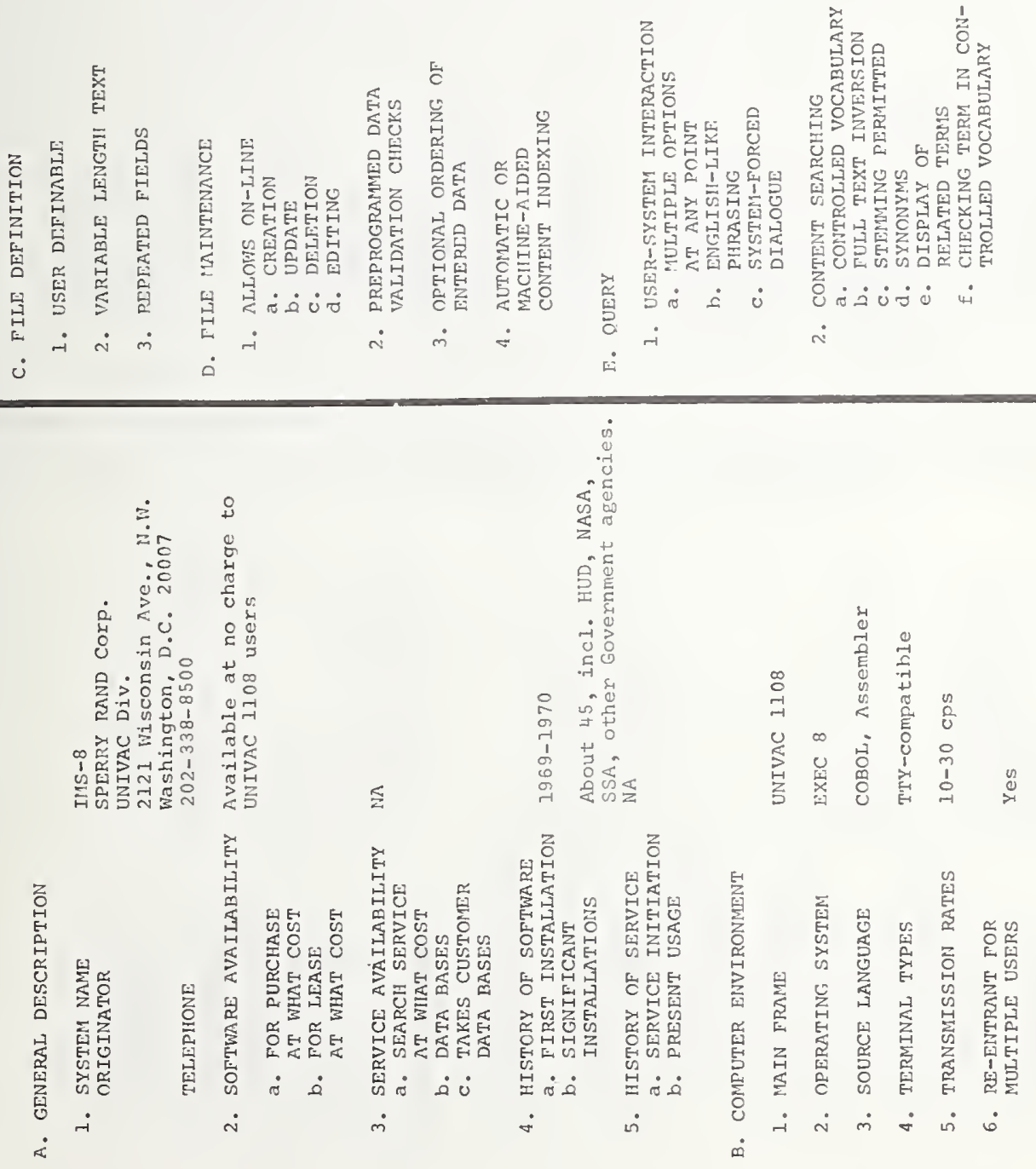


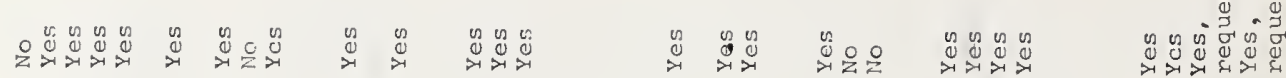

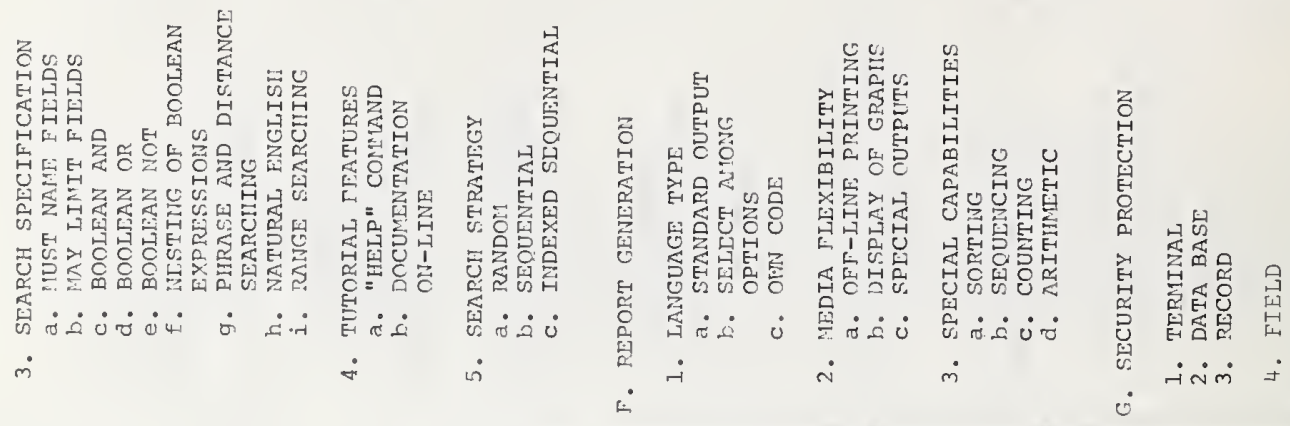
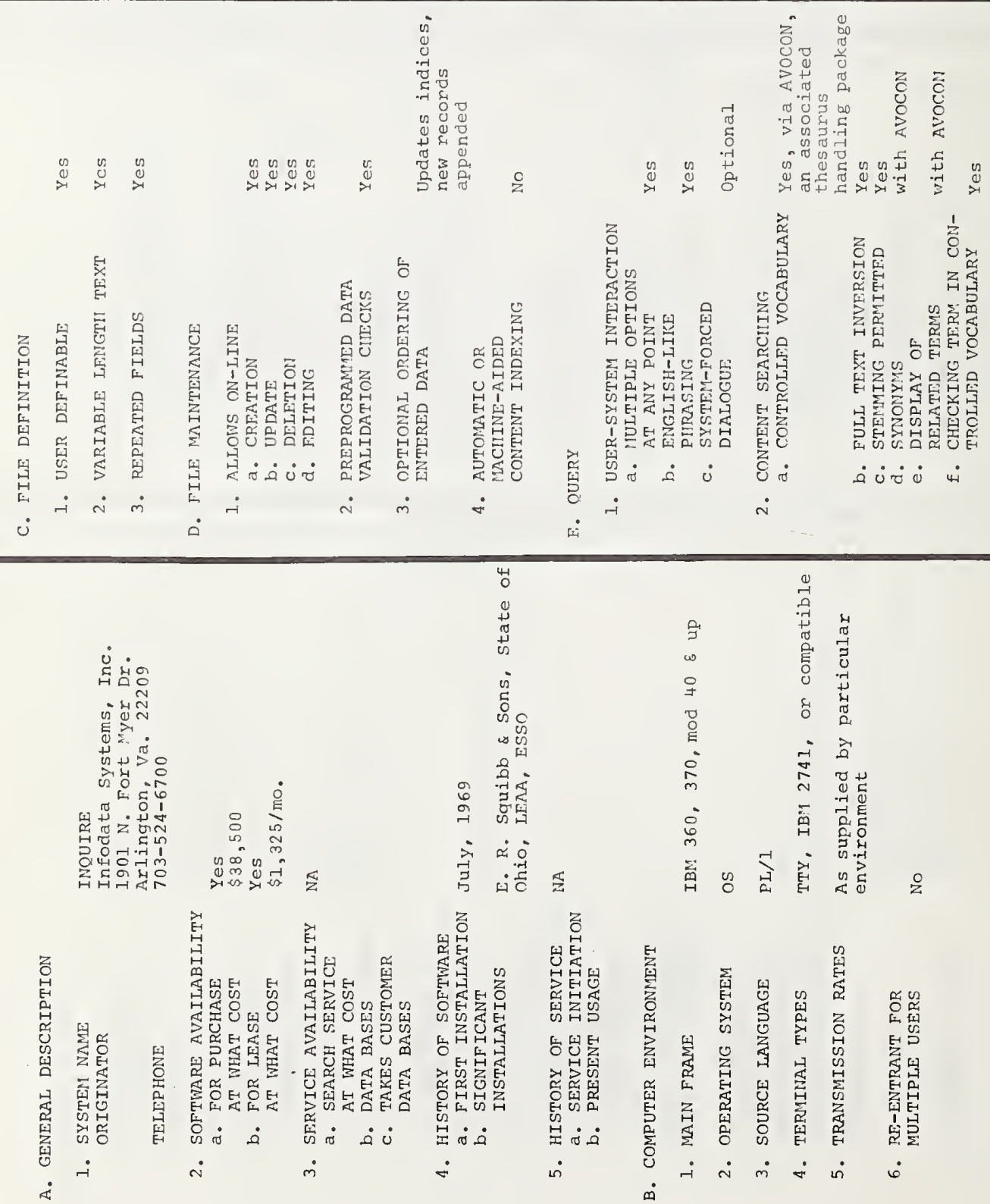


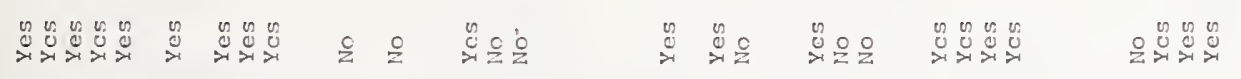

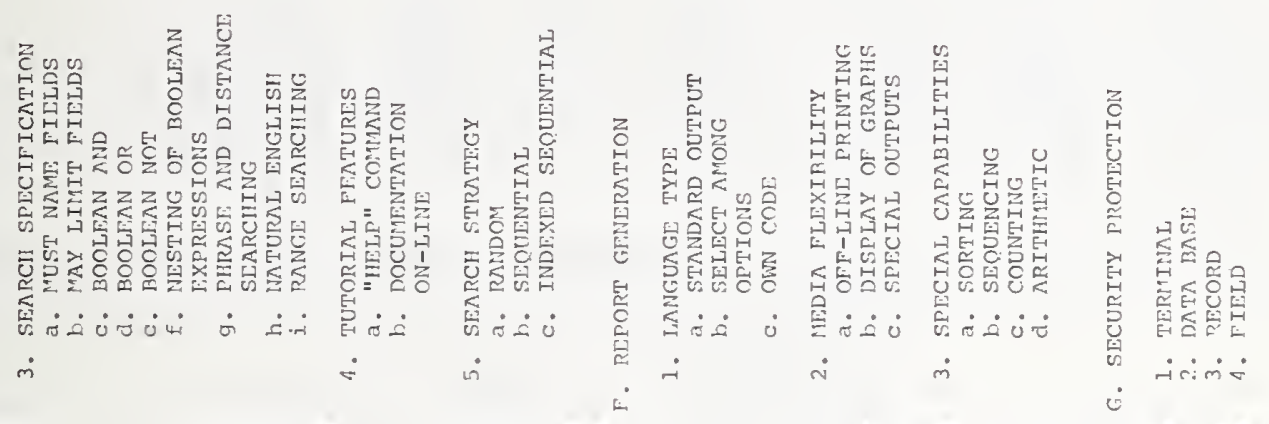

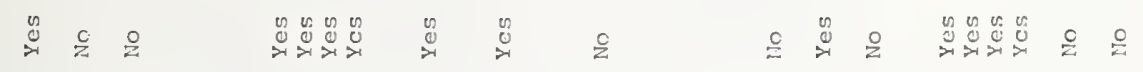

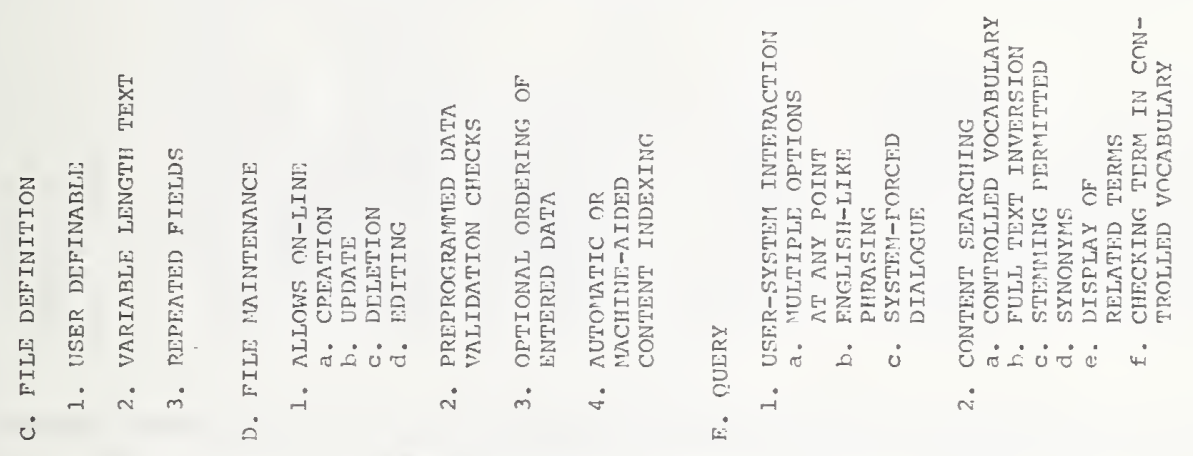

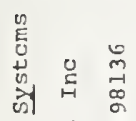

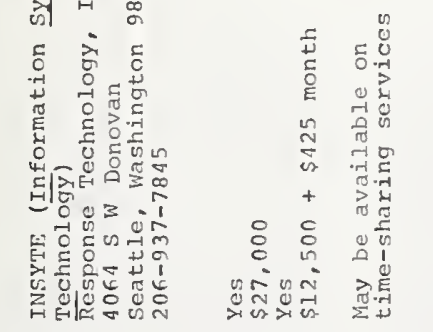

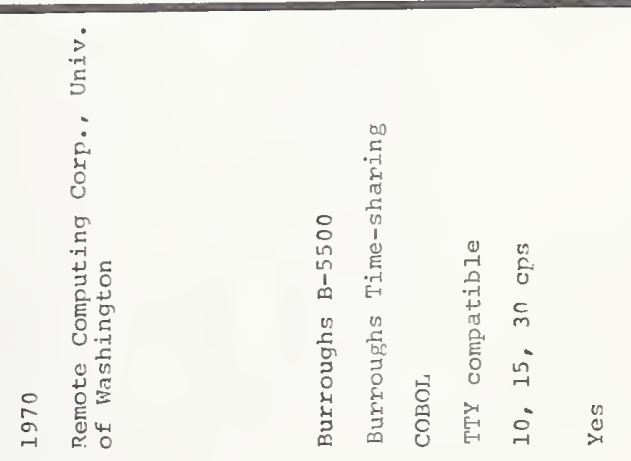

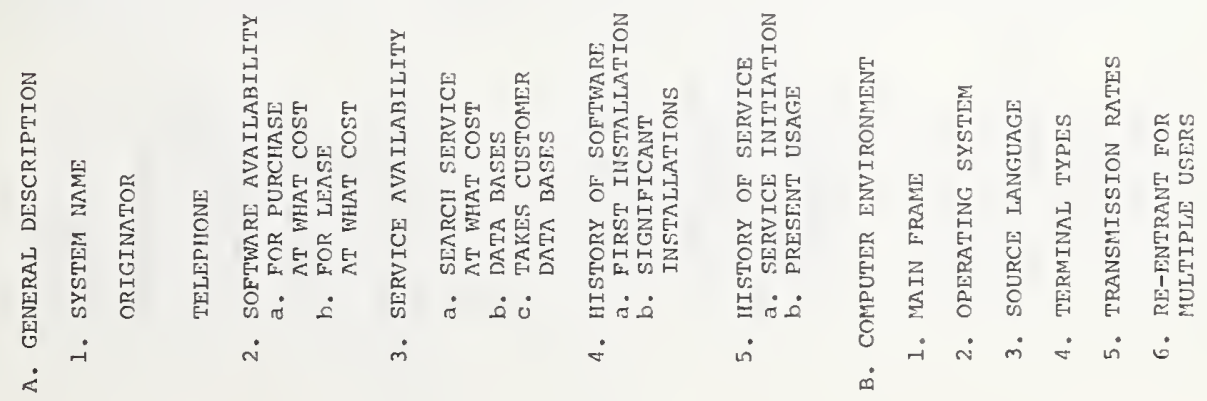



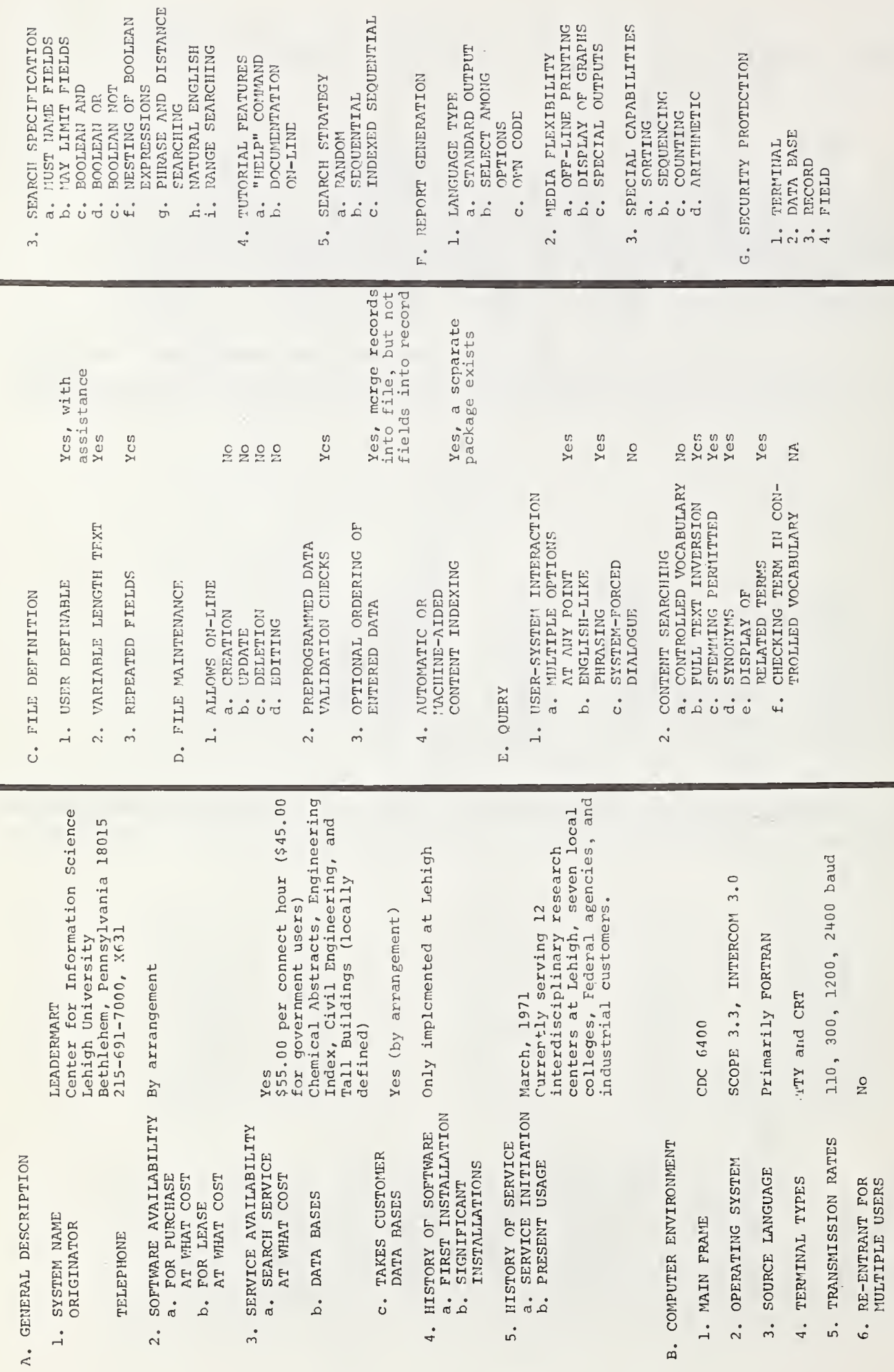


\section{$\overrightarrow{2} \frac{0}{0}=\frac{0}{0}$}

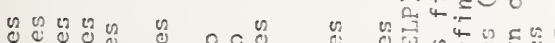

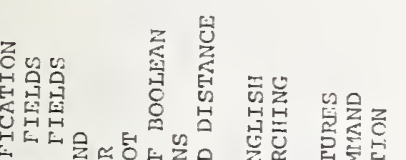

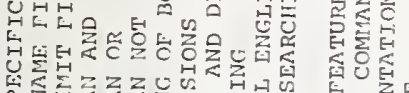

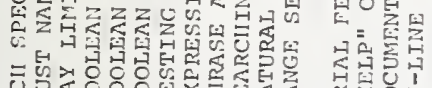

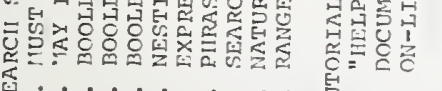

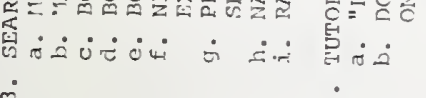

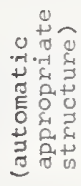

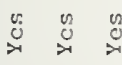

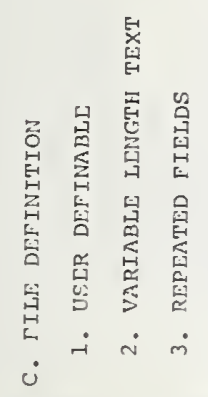

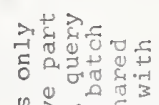

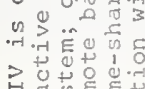

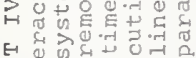

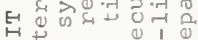

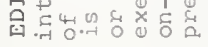

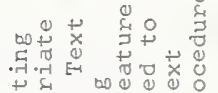

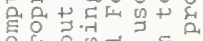

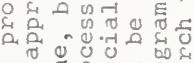

ص
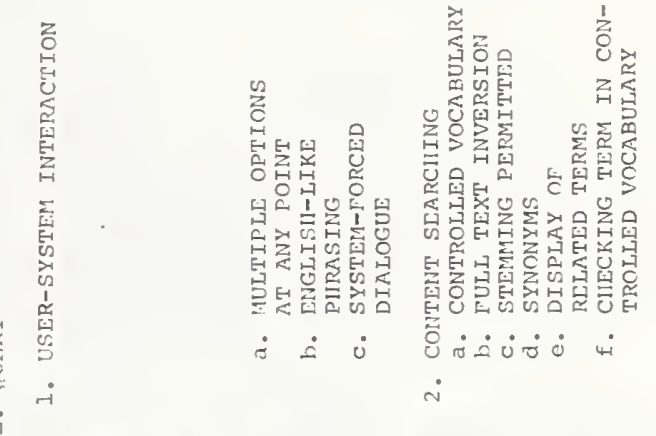

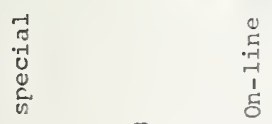

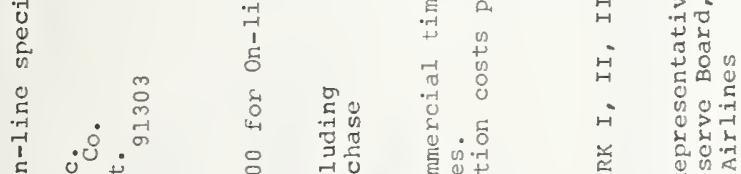

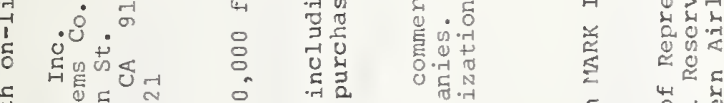

मे

>0

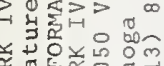

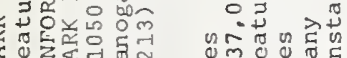

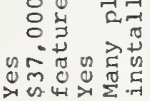

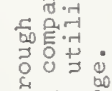

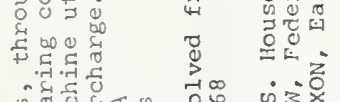

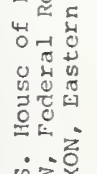

党离

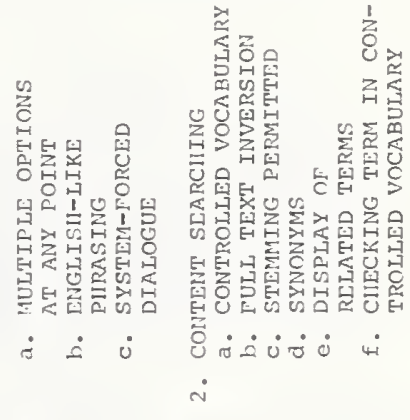

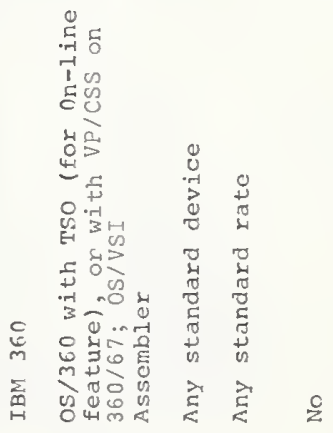

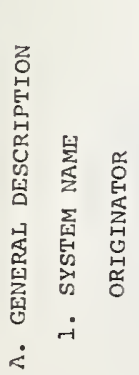

\section{窇出}

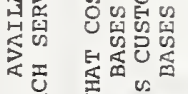

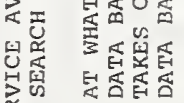

葛。
象总

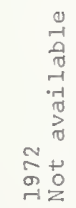

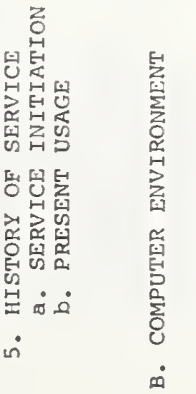

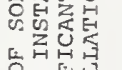

鼠淟

的的

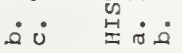

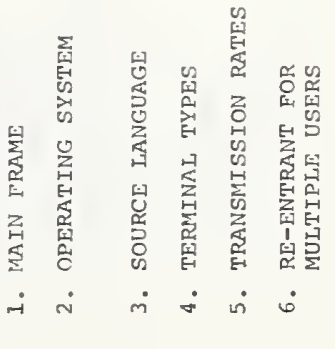


它

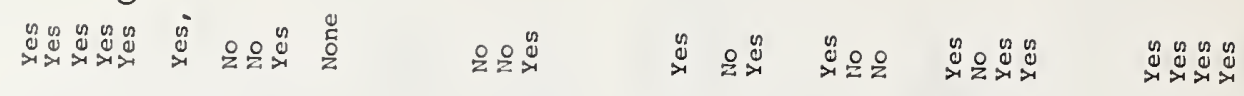

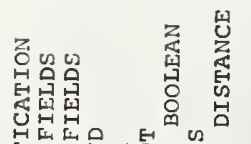

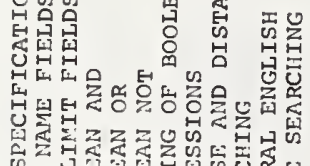

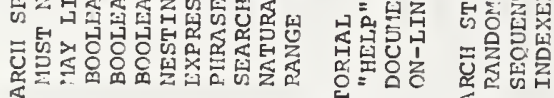

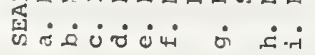

$\dot{m}$

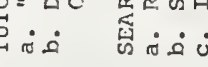

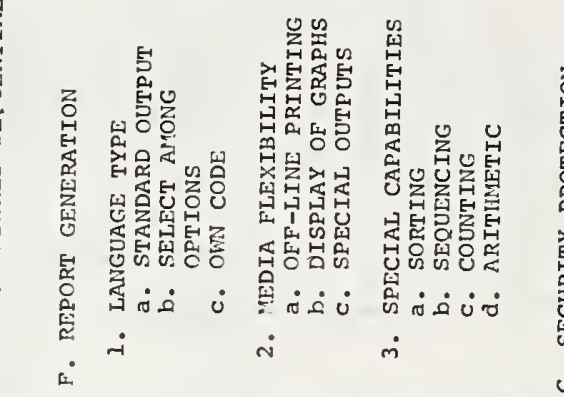

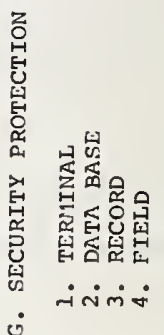

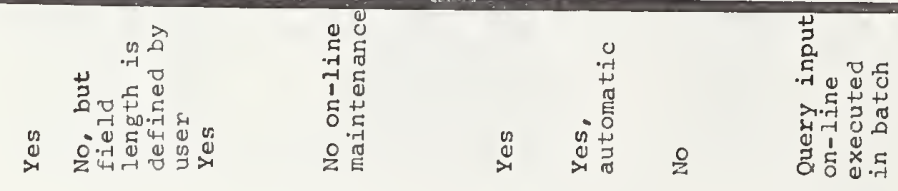
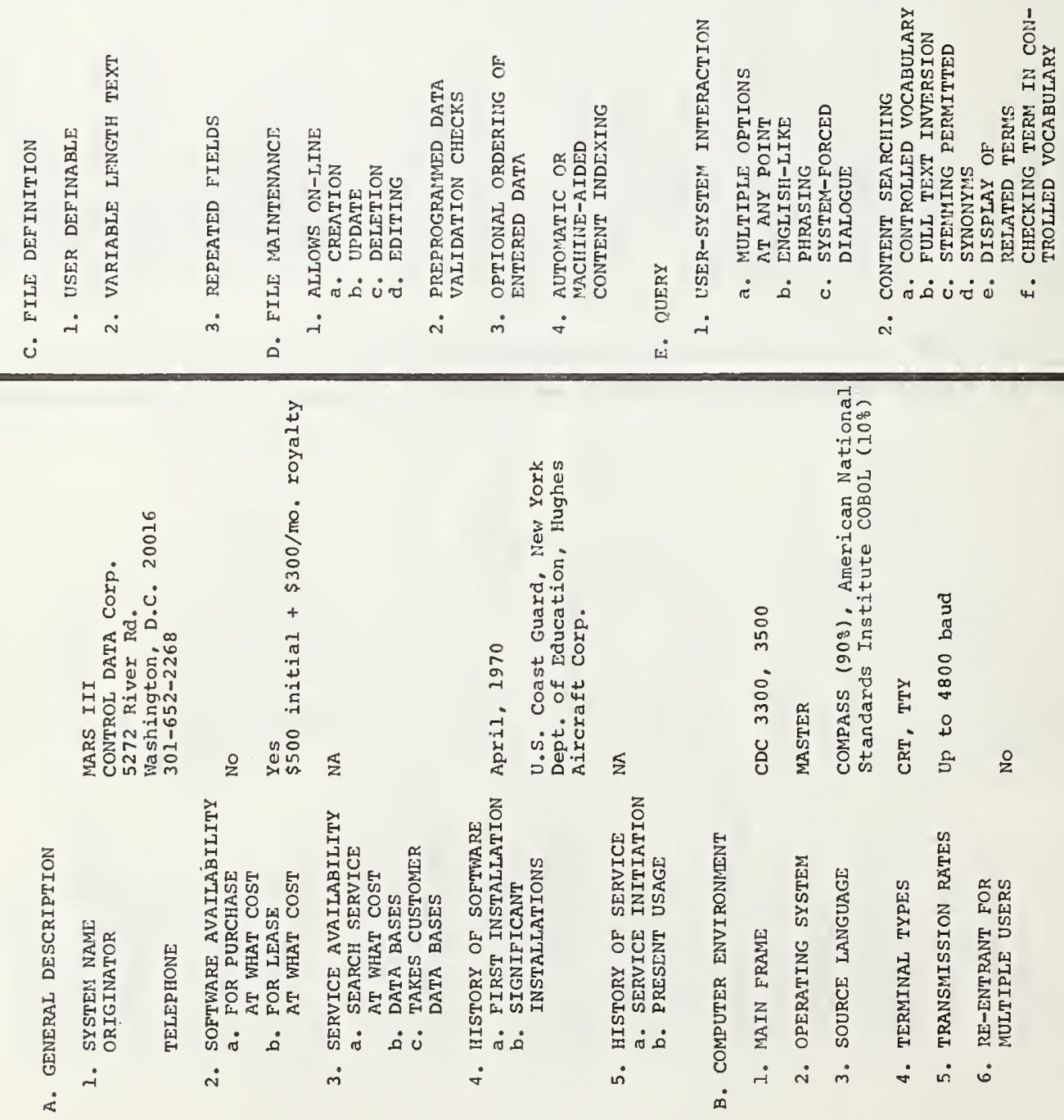


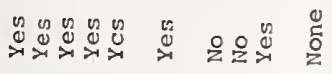
있

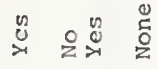

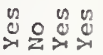

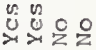

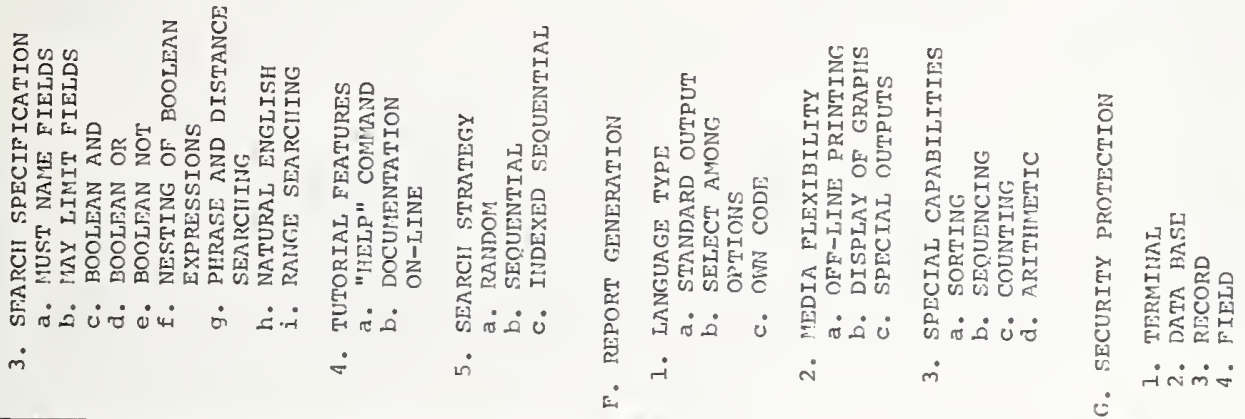

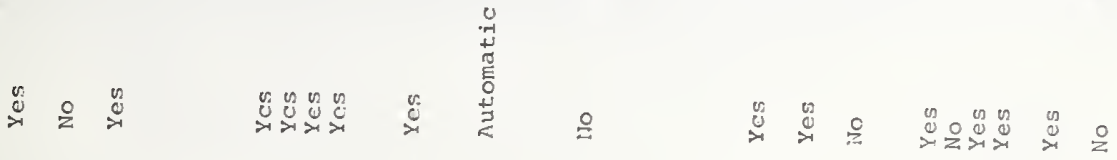
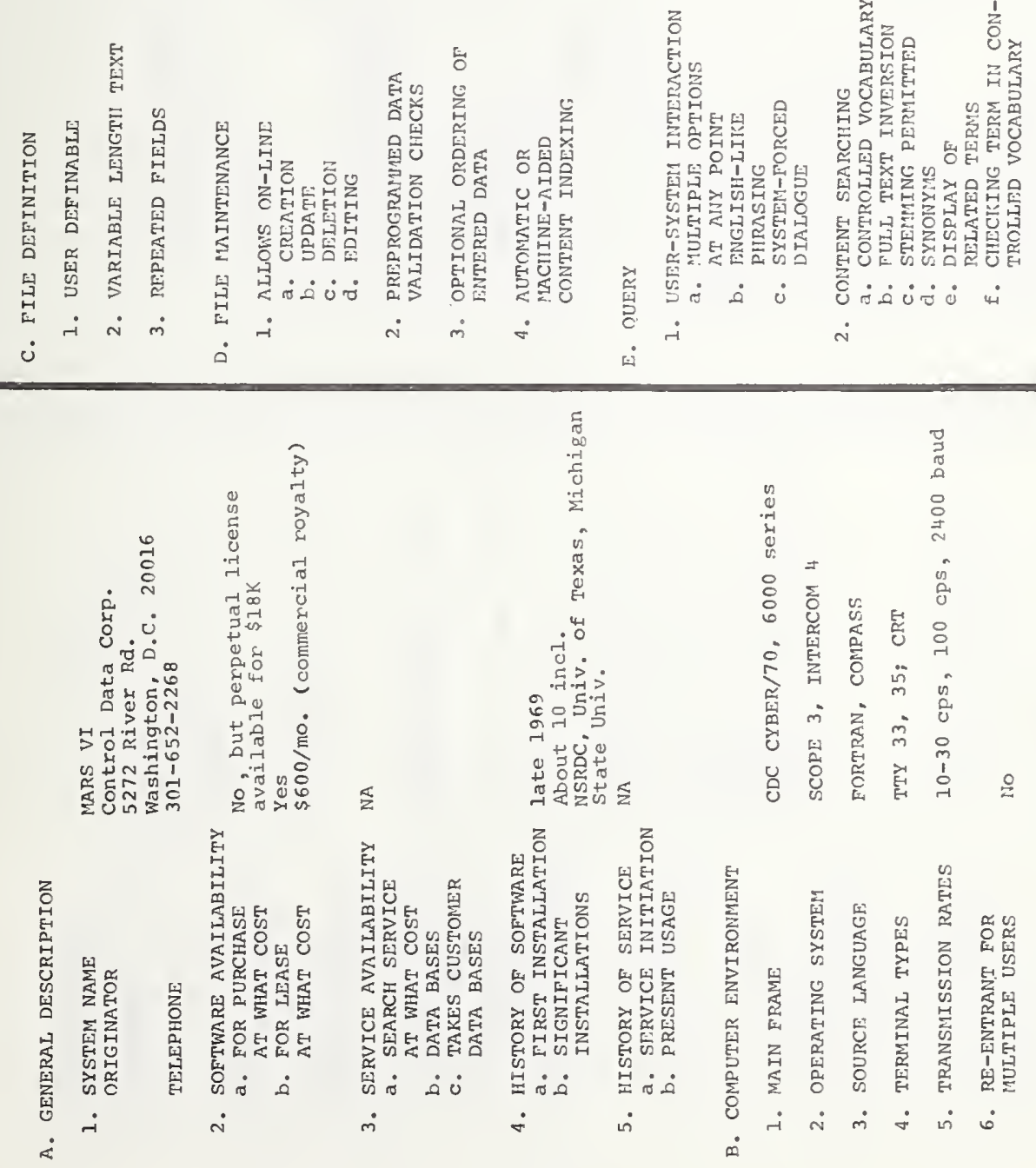


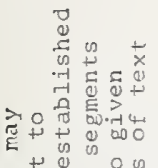

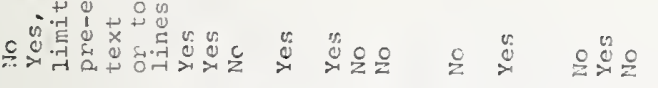

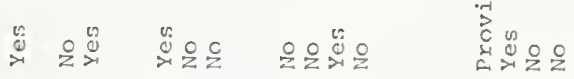

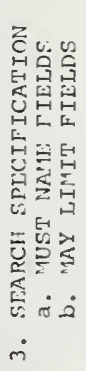

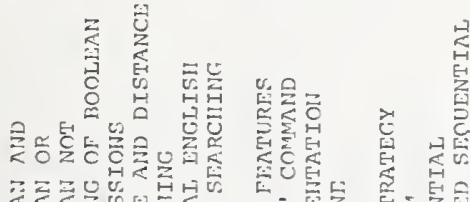

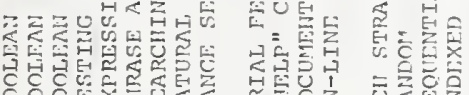

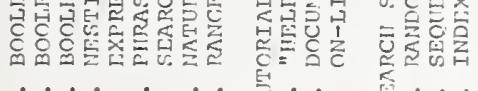

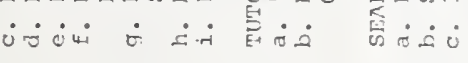

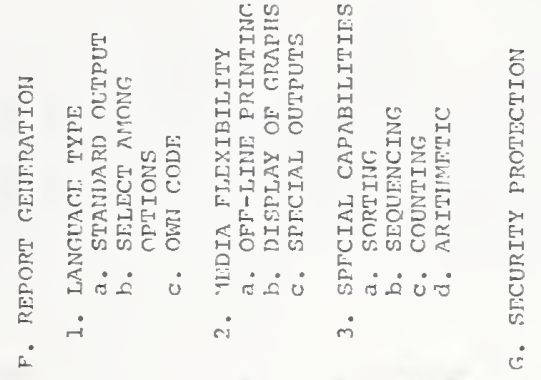

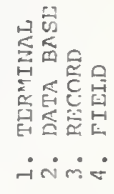

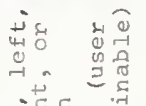

害
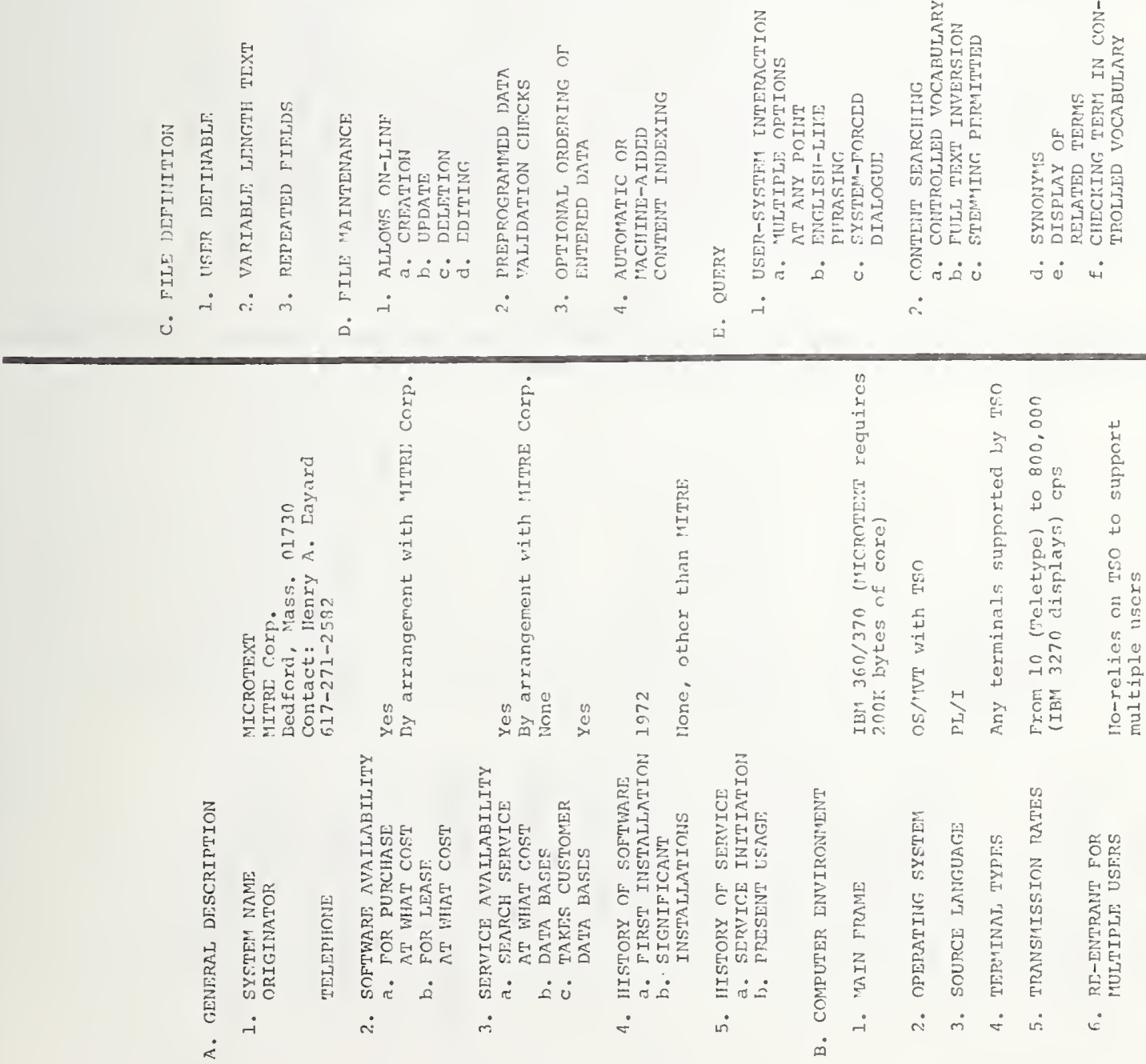


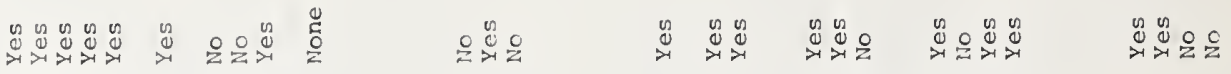

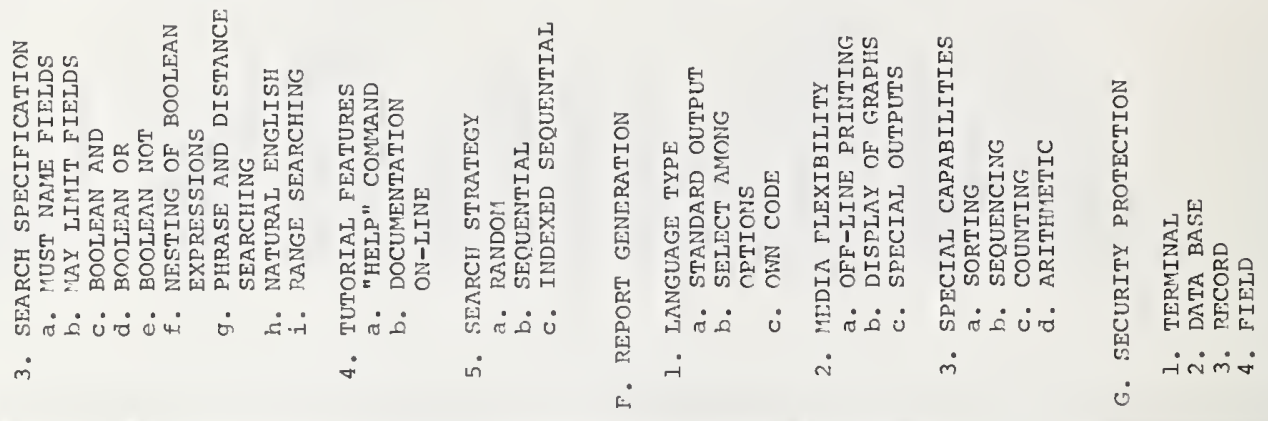

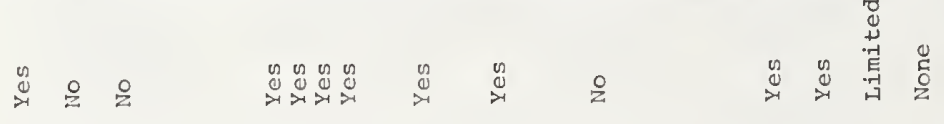

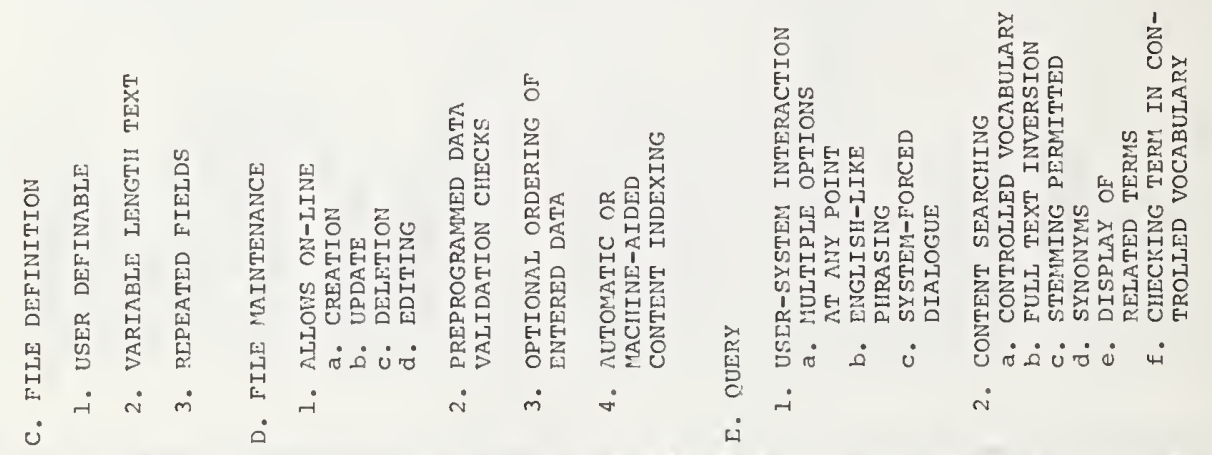

in
on
in
0
0

I

敢

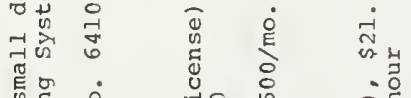

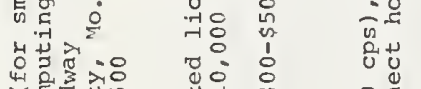

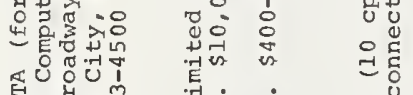

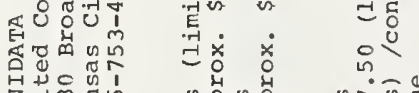

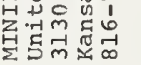

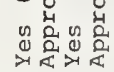

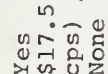

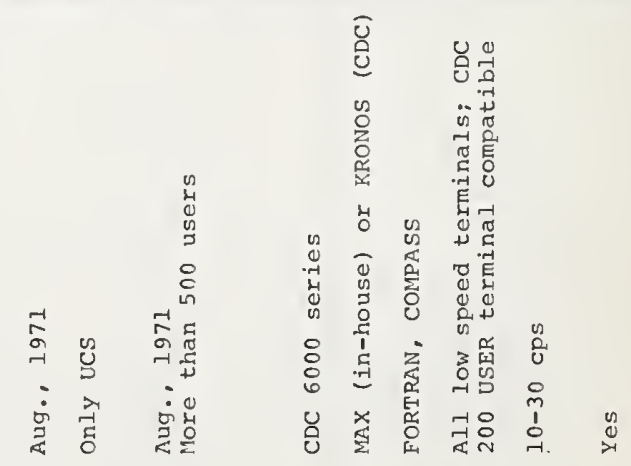

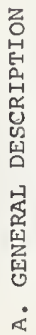

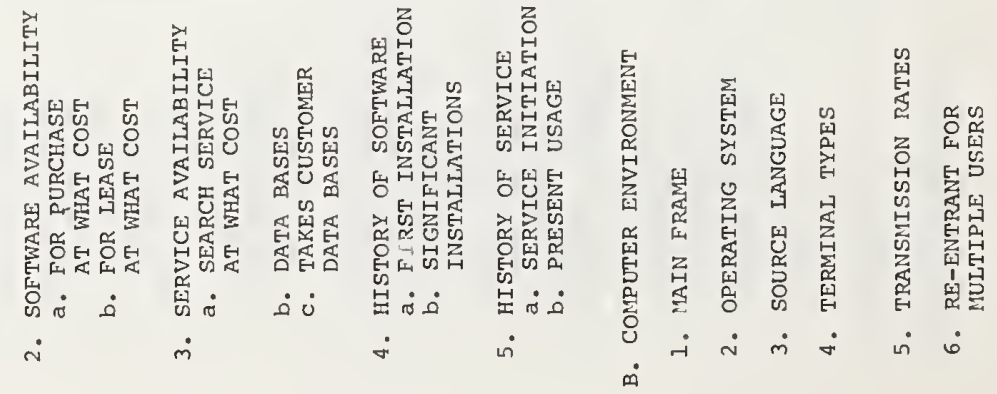


g.

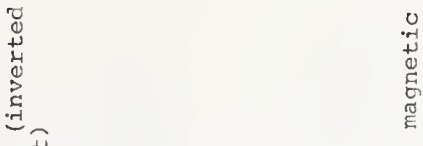

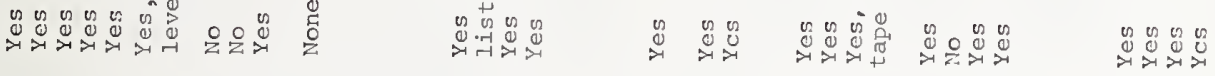
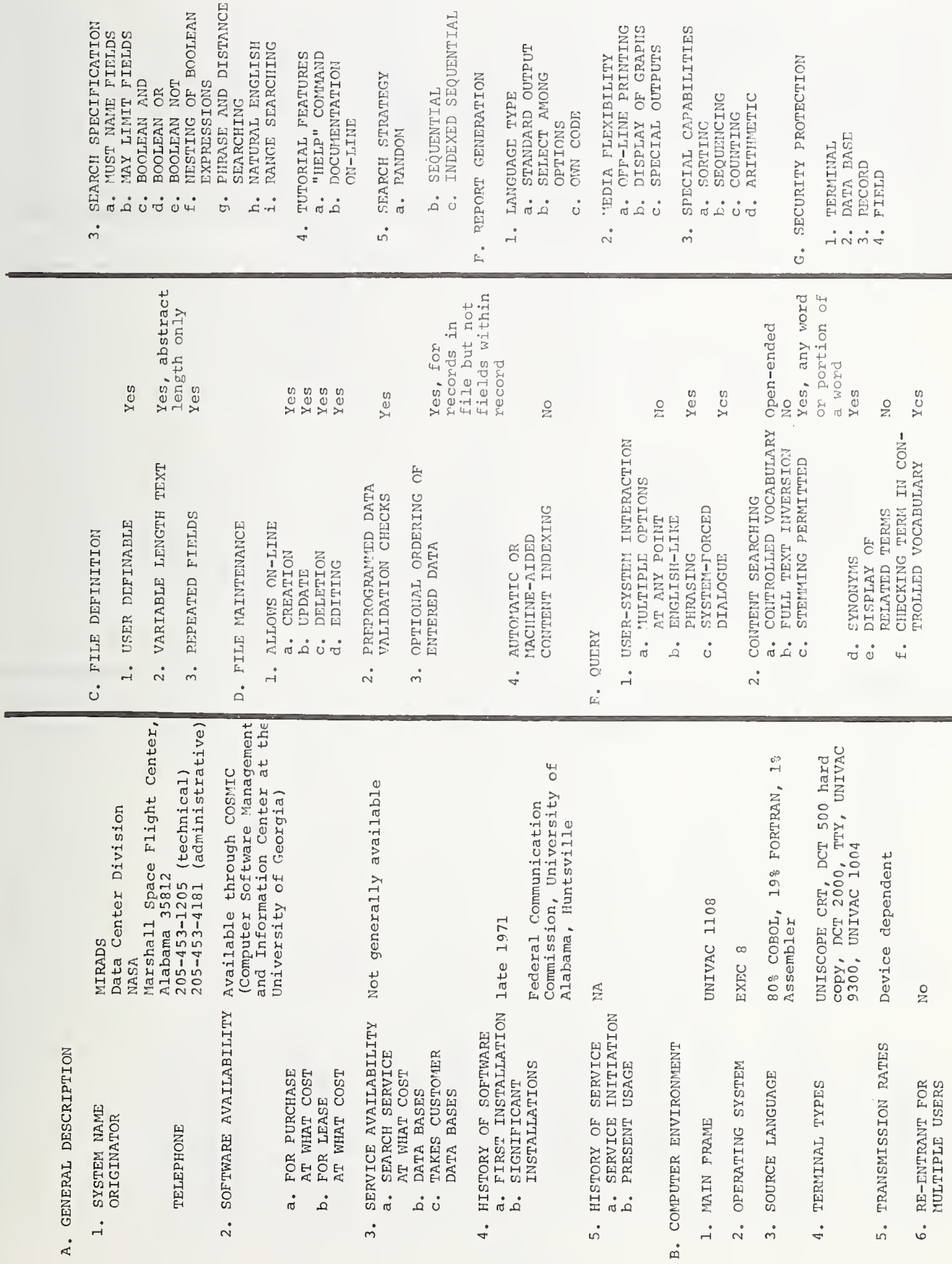


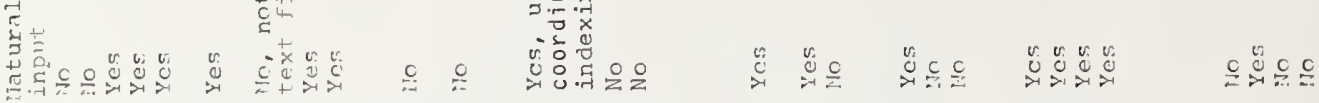
能童

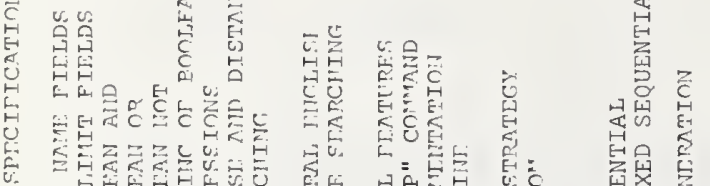

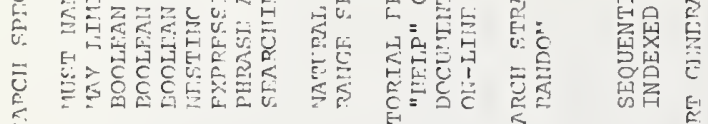

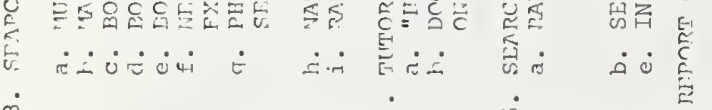

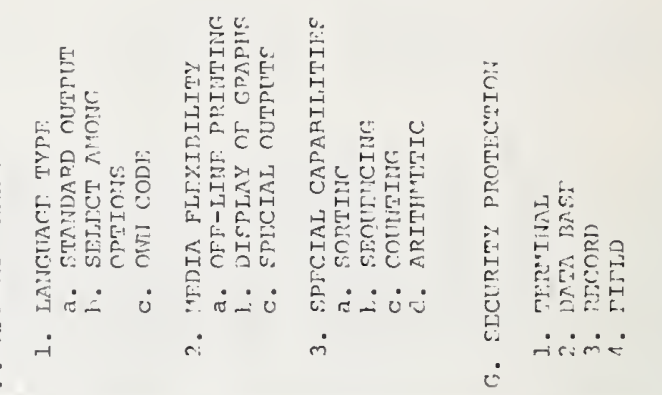

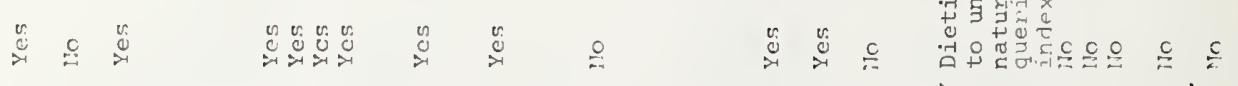
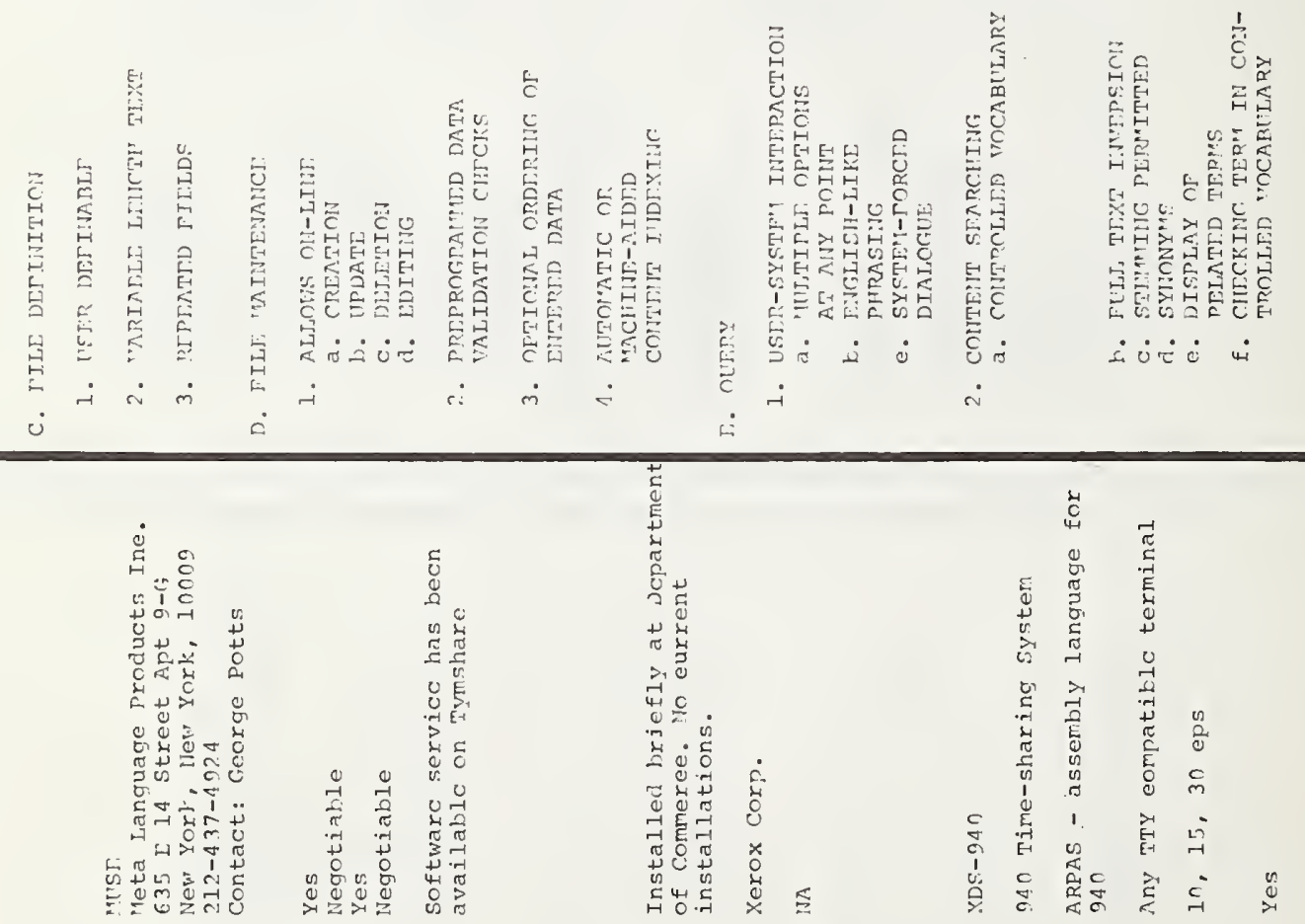

zo

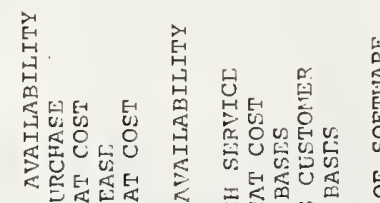
至

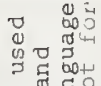

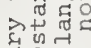

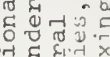




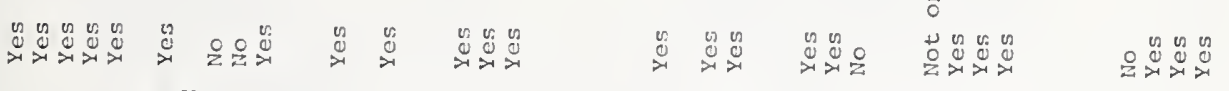

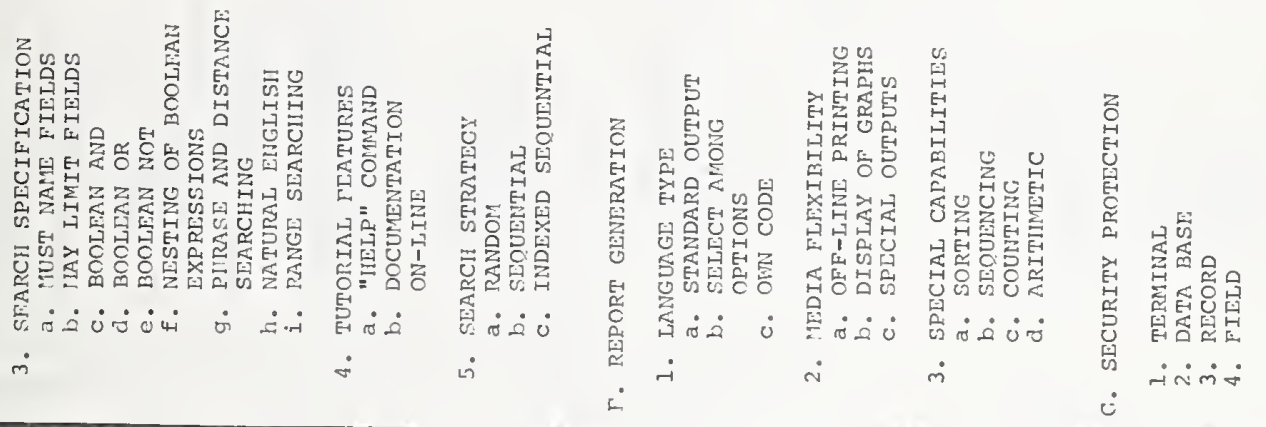

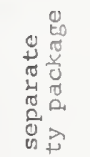

荧
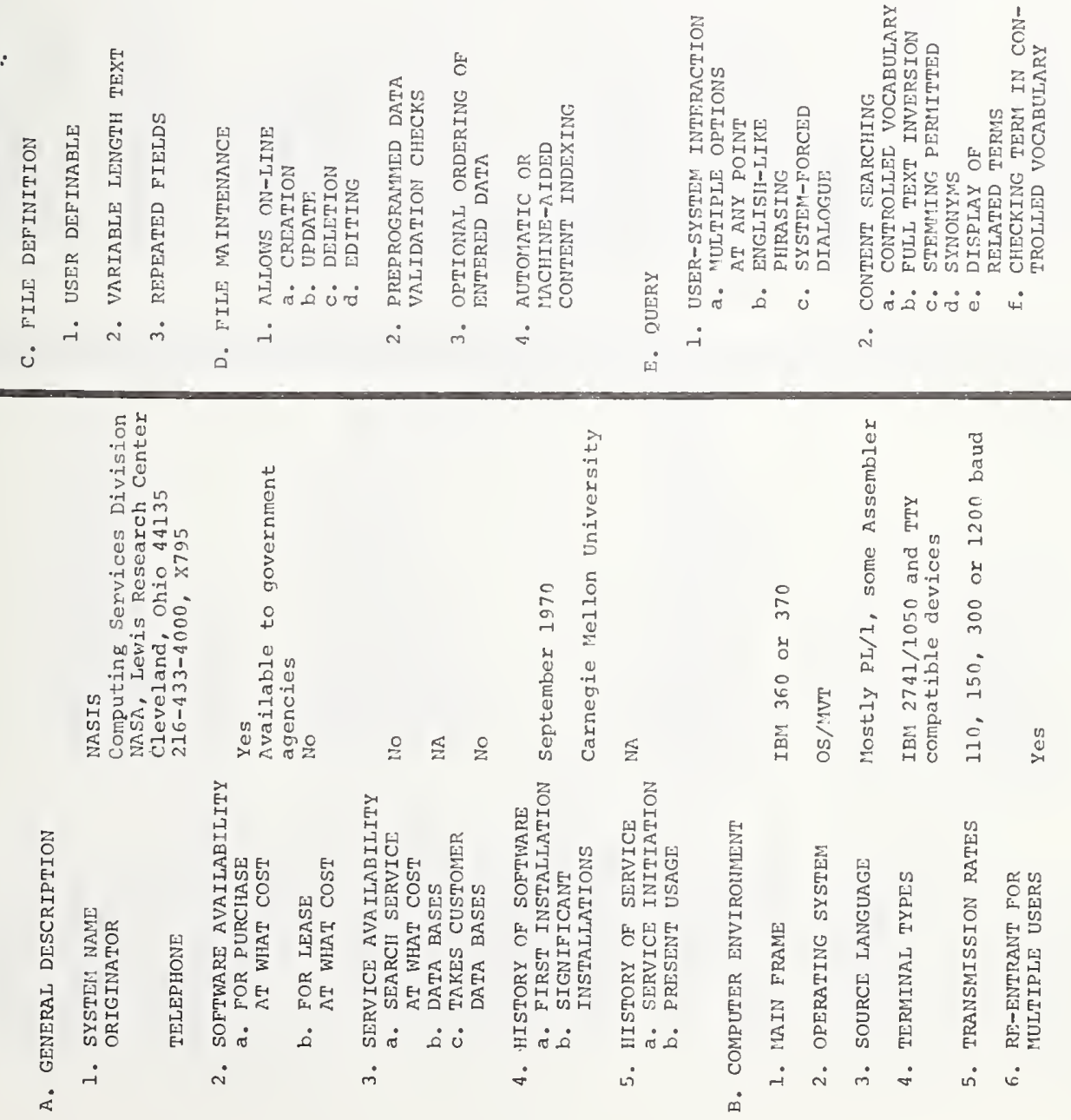


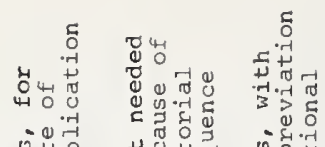

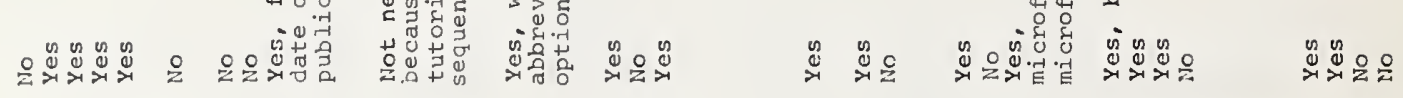

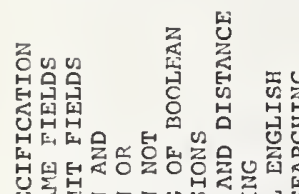

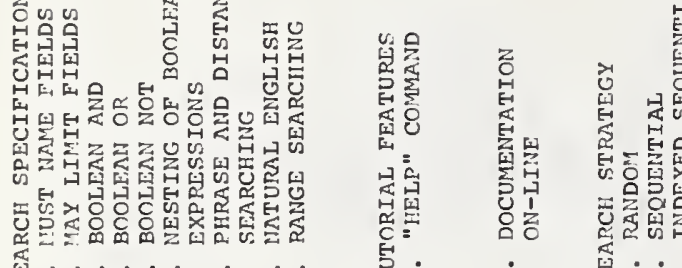

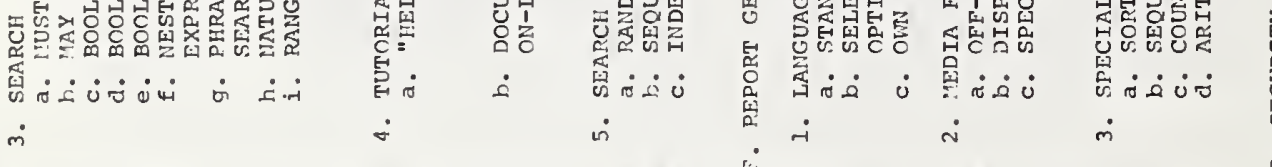

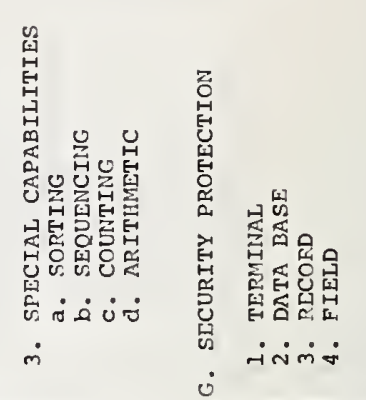

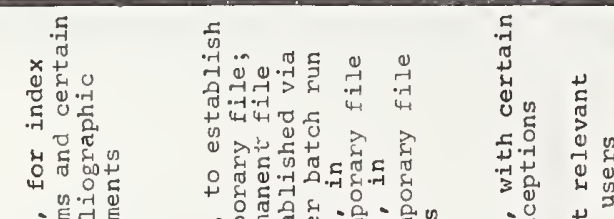

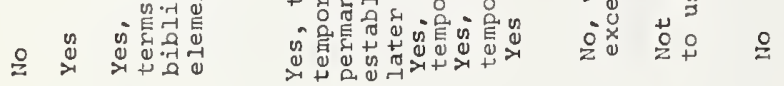

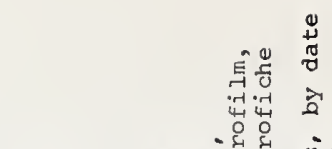




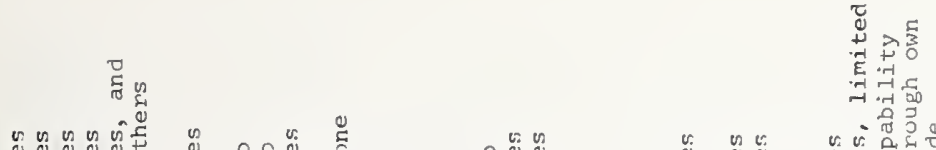

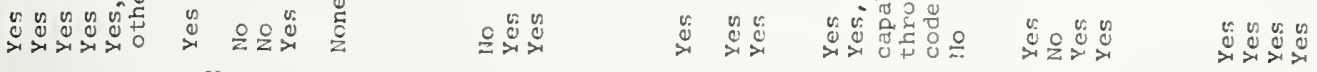

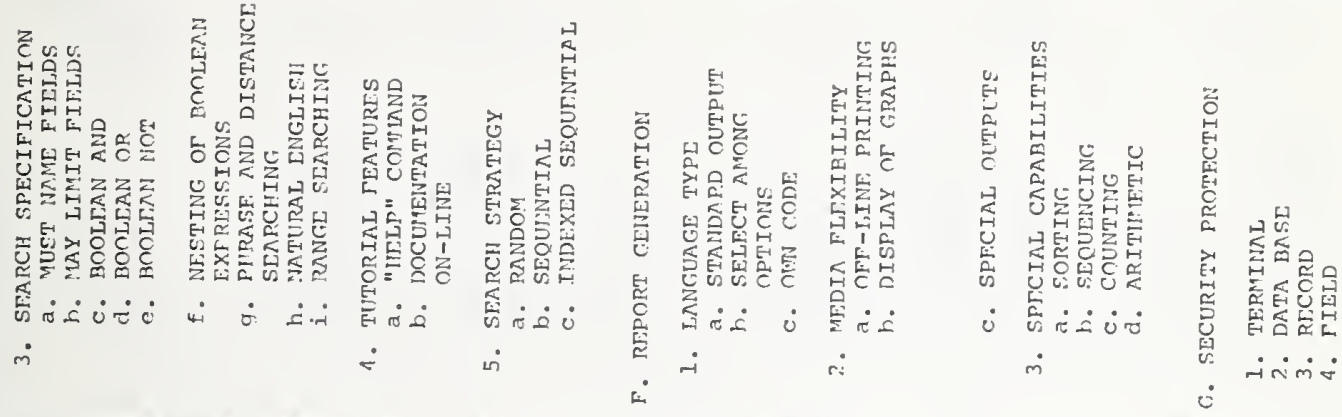

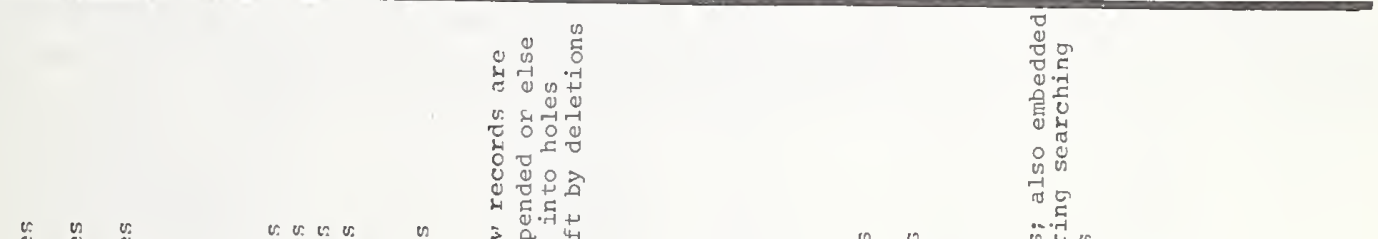

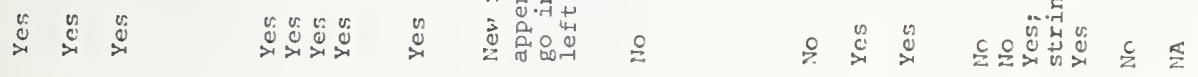

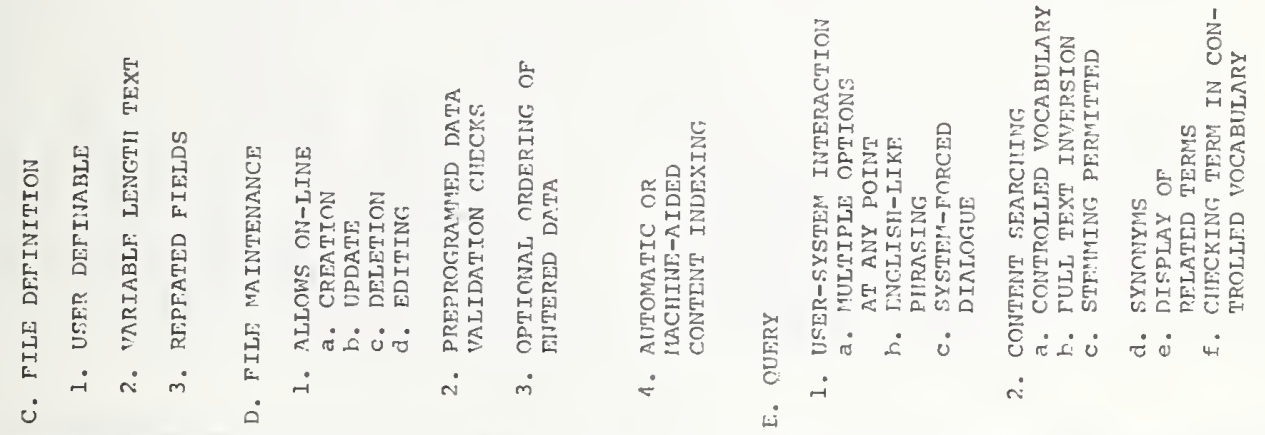

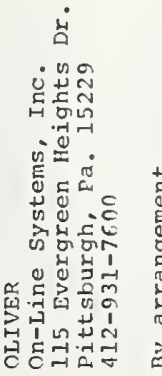
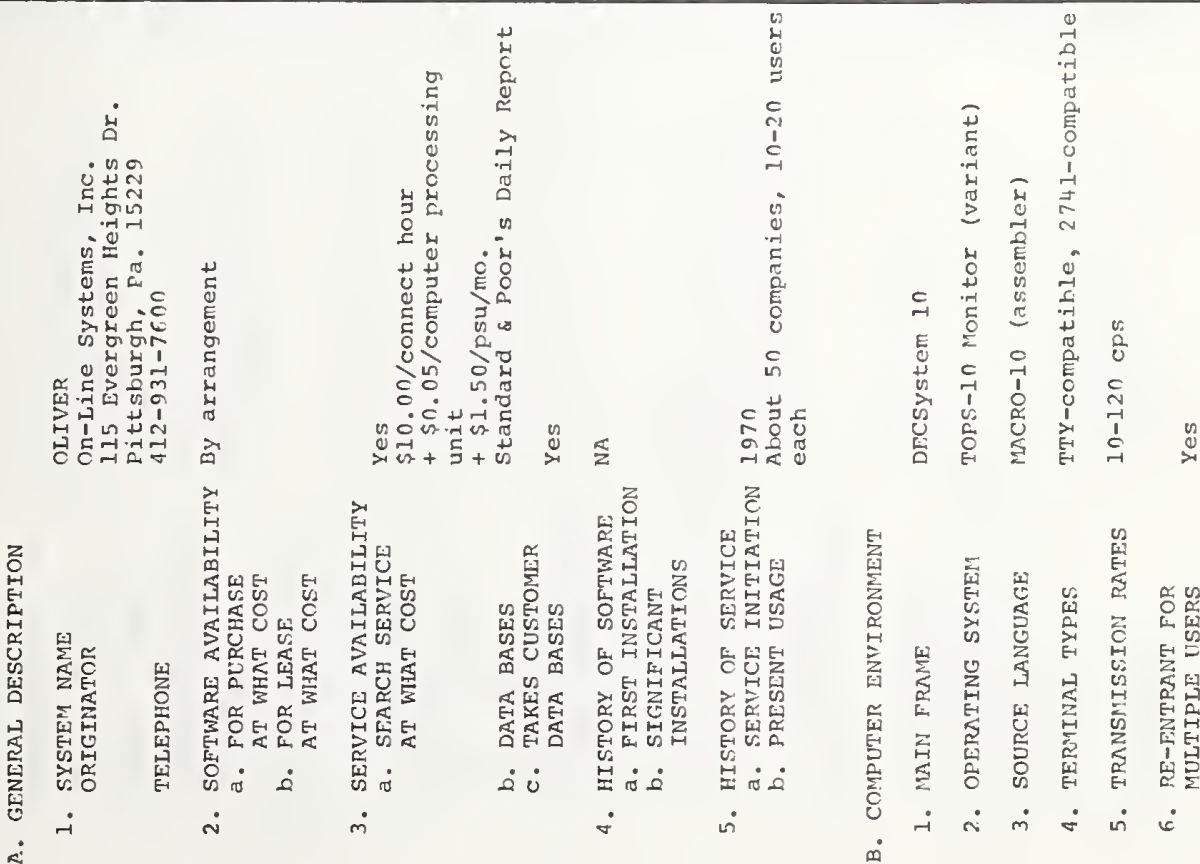

舅

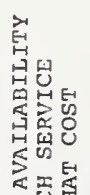

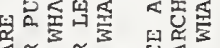

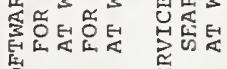

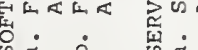

$\dot{\sim}$

$\dot{m}$
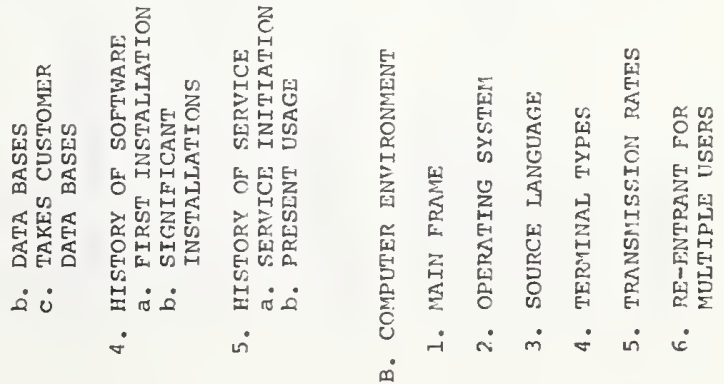


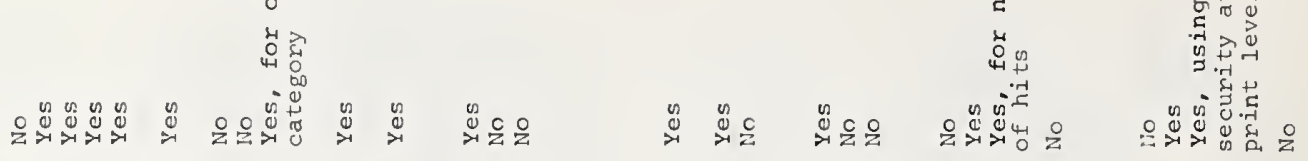

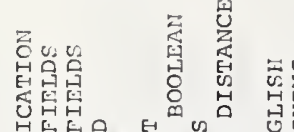

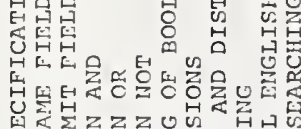

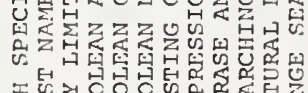

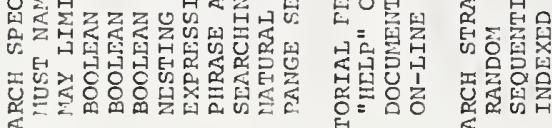

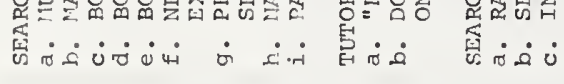
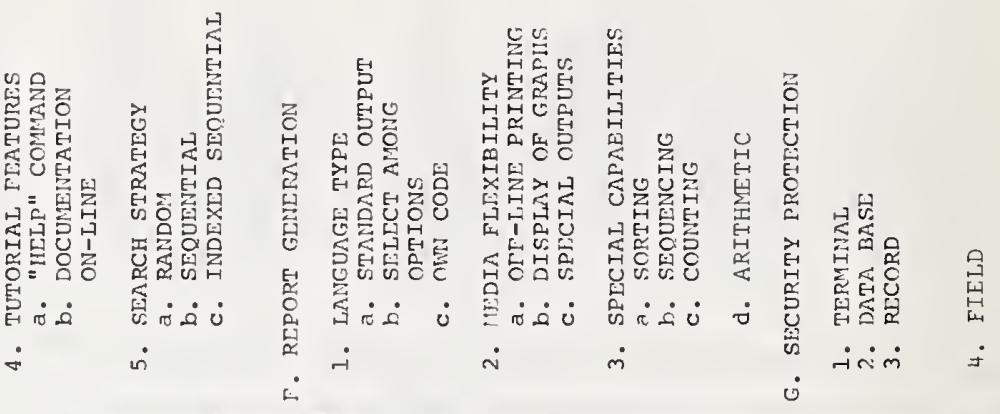

新

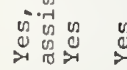
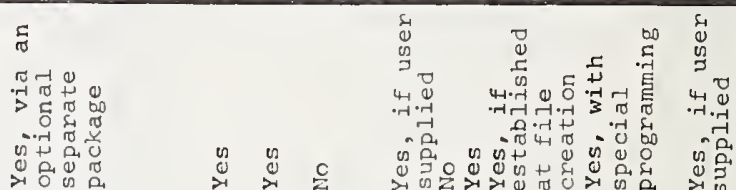

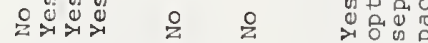

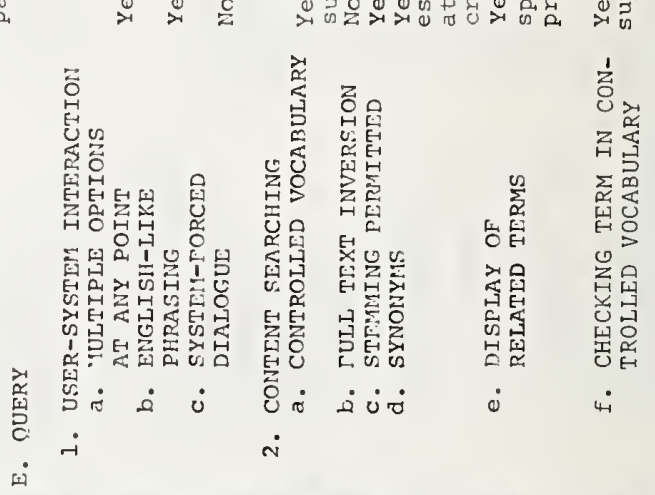

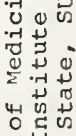

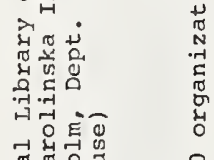

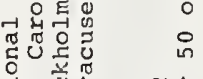

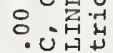

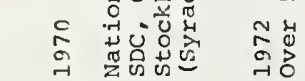

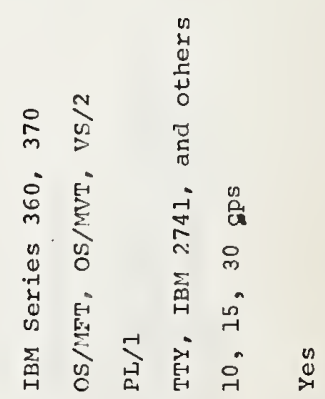

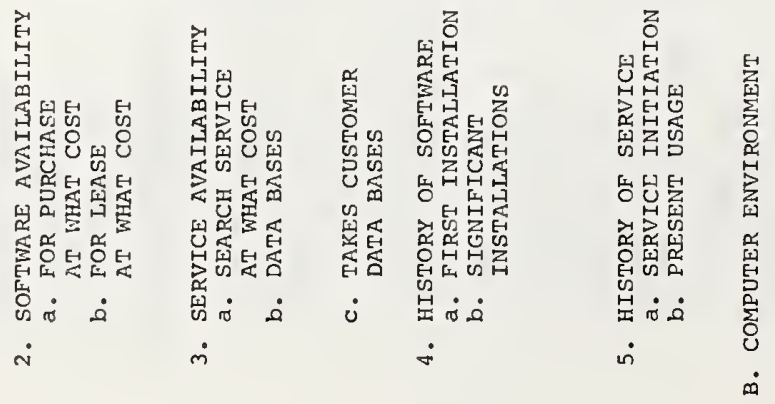

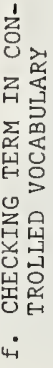

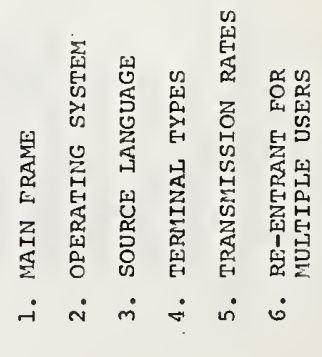




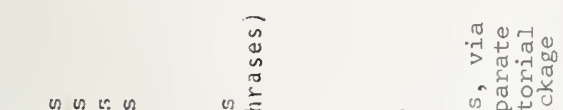

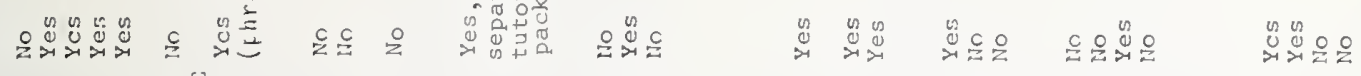

蒙品

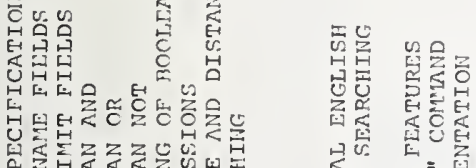

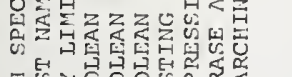

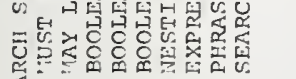

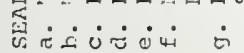

$\dot{m}$

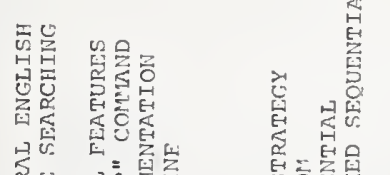

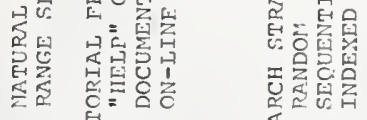

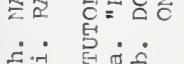

$\dot{\square}$

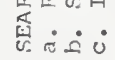

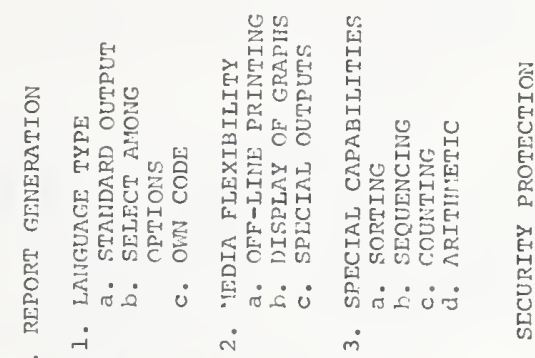

窟岕

产空品

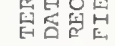

तिंक्ष

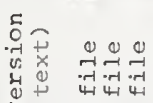 \\ 穿

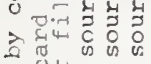

ะั ะ

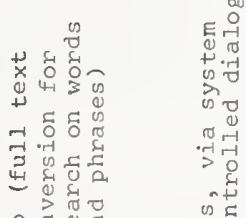

\begin{tabular}{l}
5 \\
5 \\
-5 \\
0 \\
0 \\
5 \\
5 \\
5 \\
\hline
\end{tabular}

is

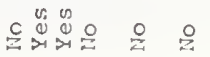

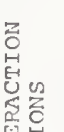
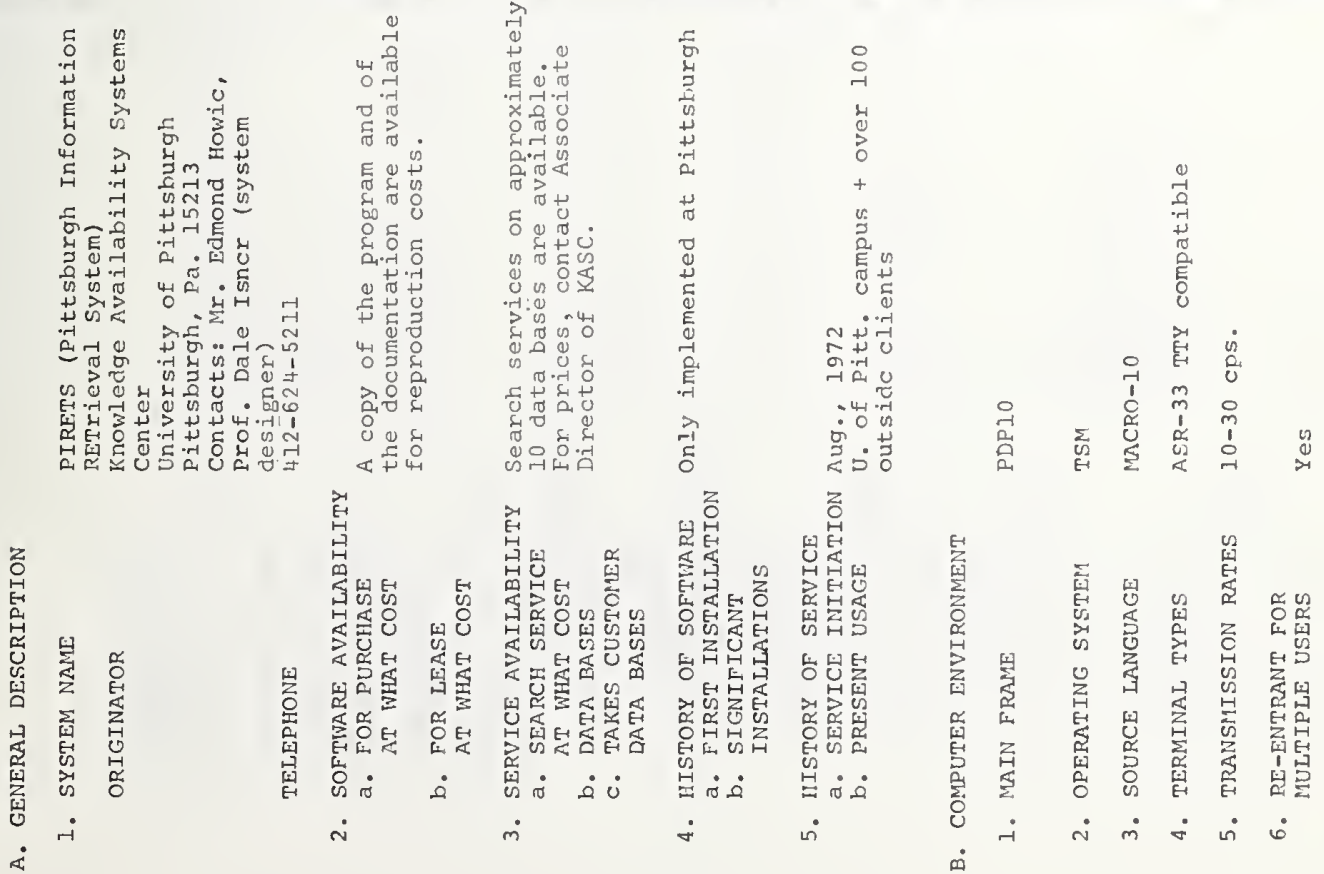

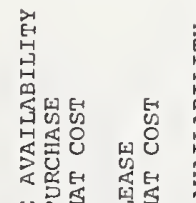

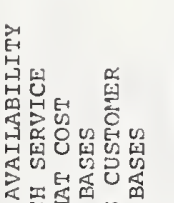

递要

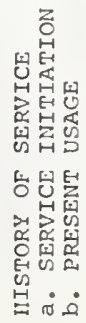

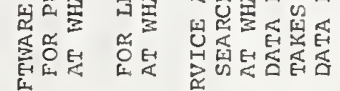

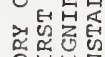

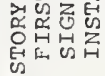

का

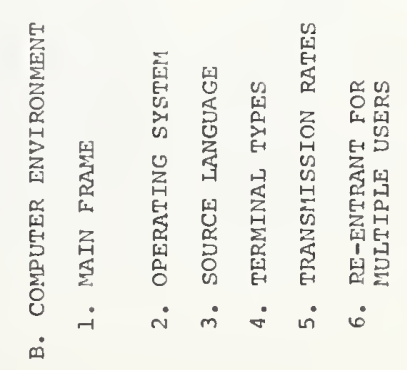



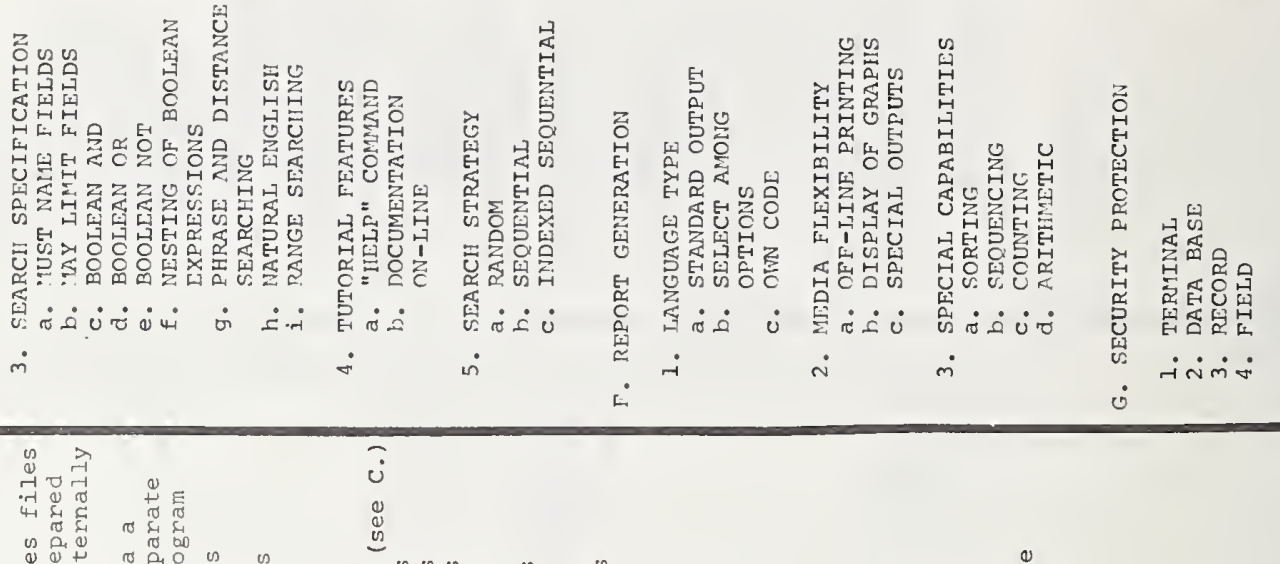

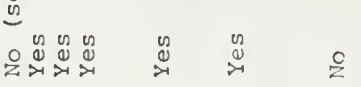

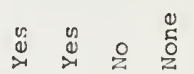
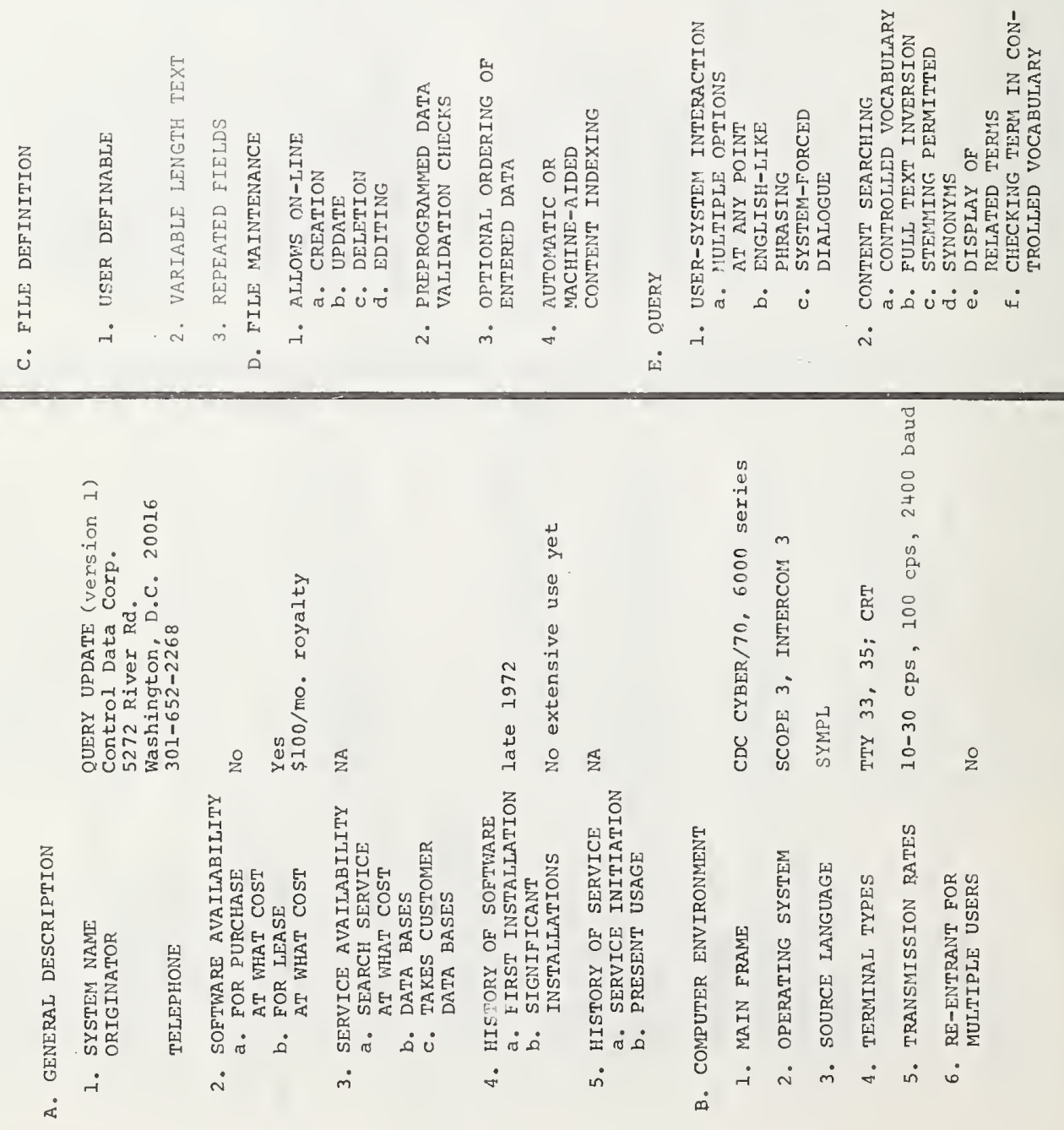


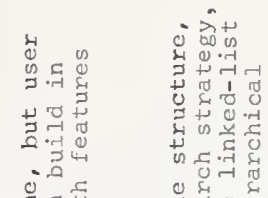

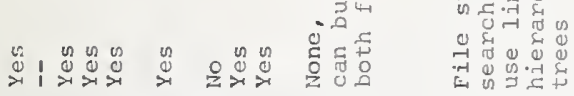

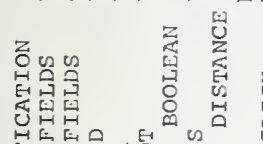

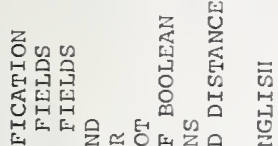

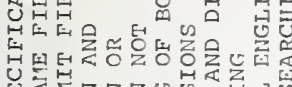

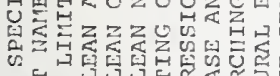

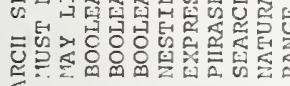
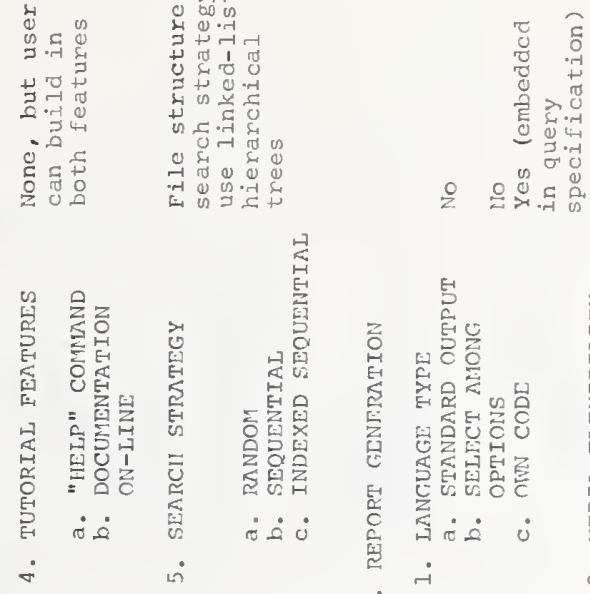

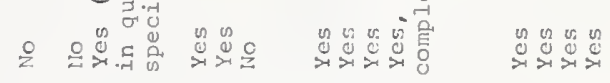

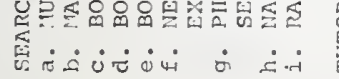

$\dot{m}$

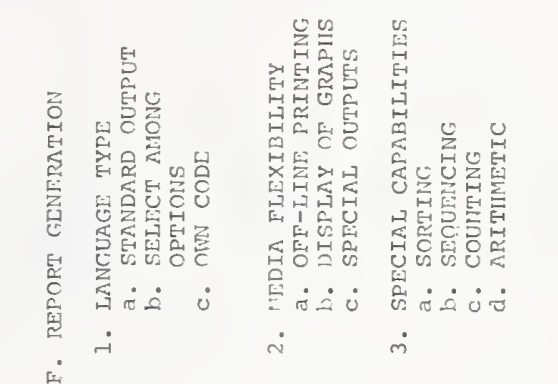

노ํํํำ

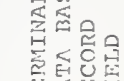

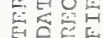

-iिंखं

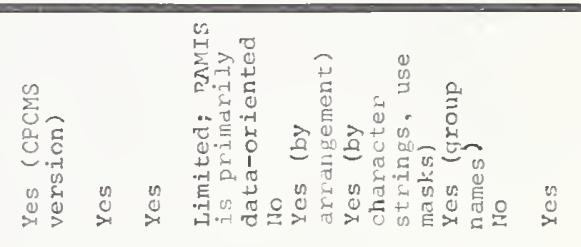

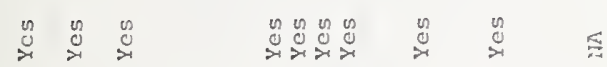

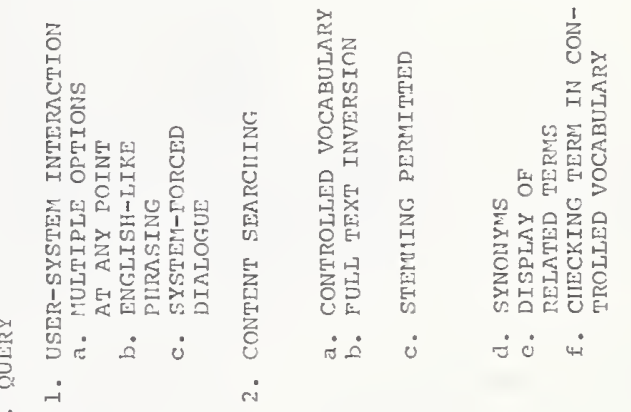

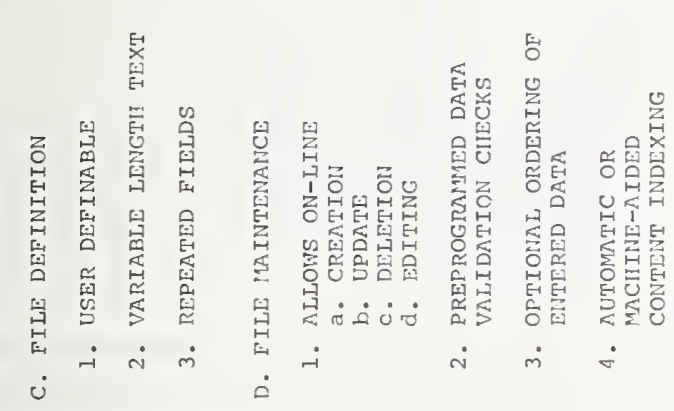

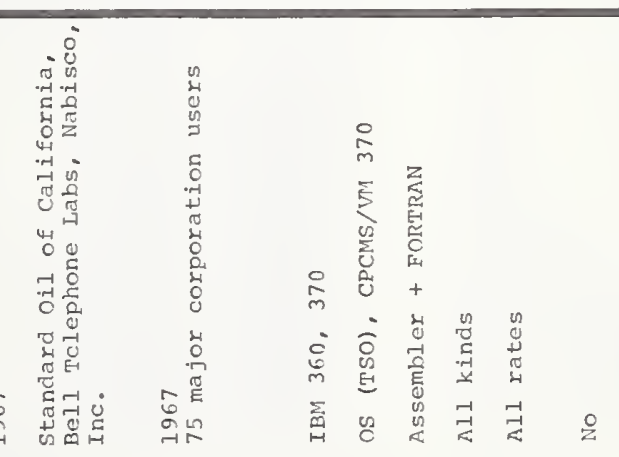

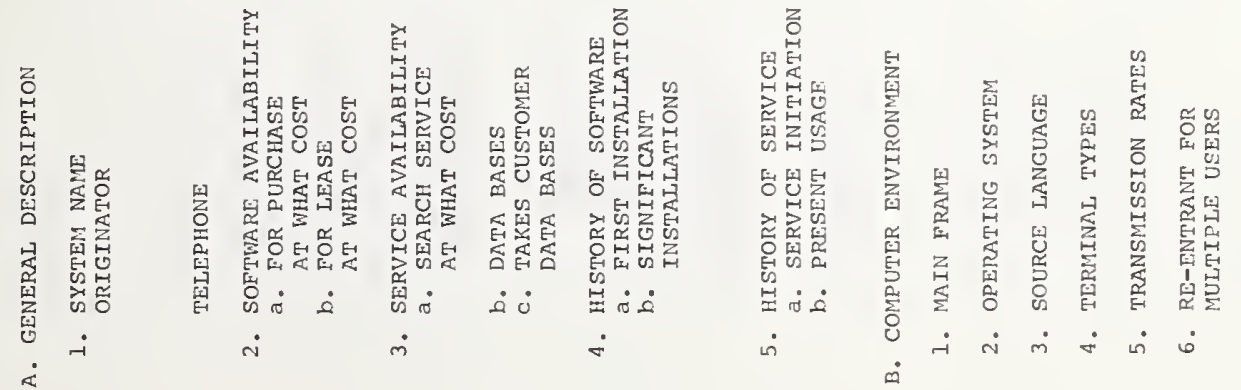


等急

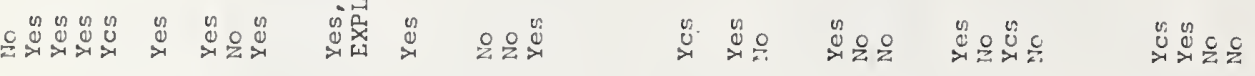

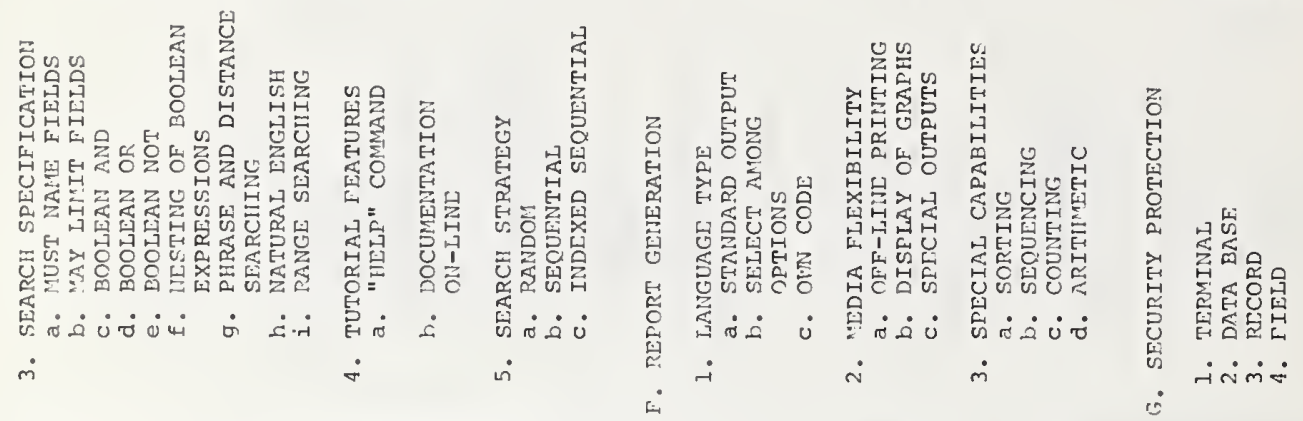

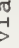

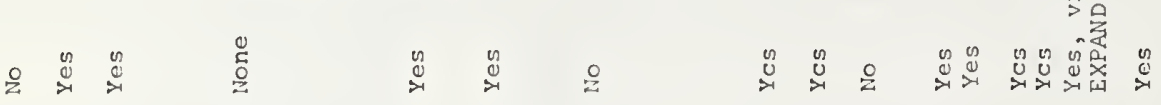

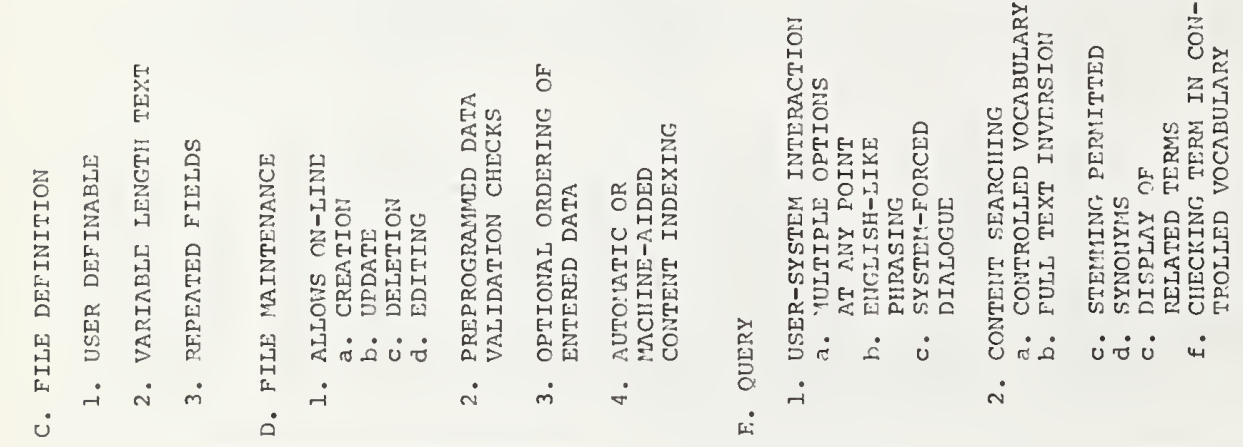

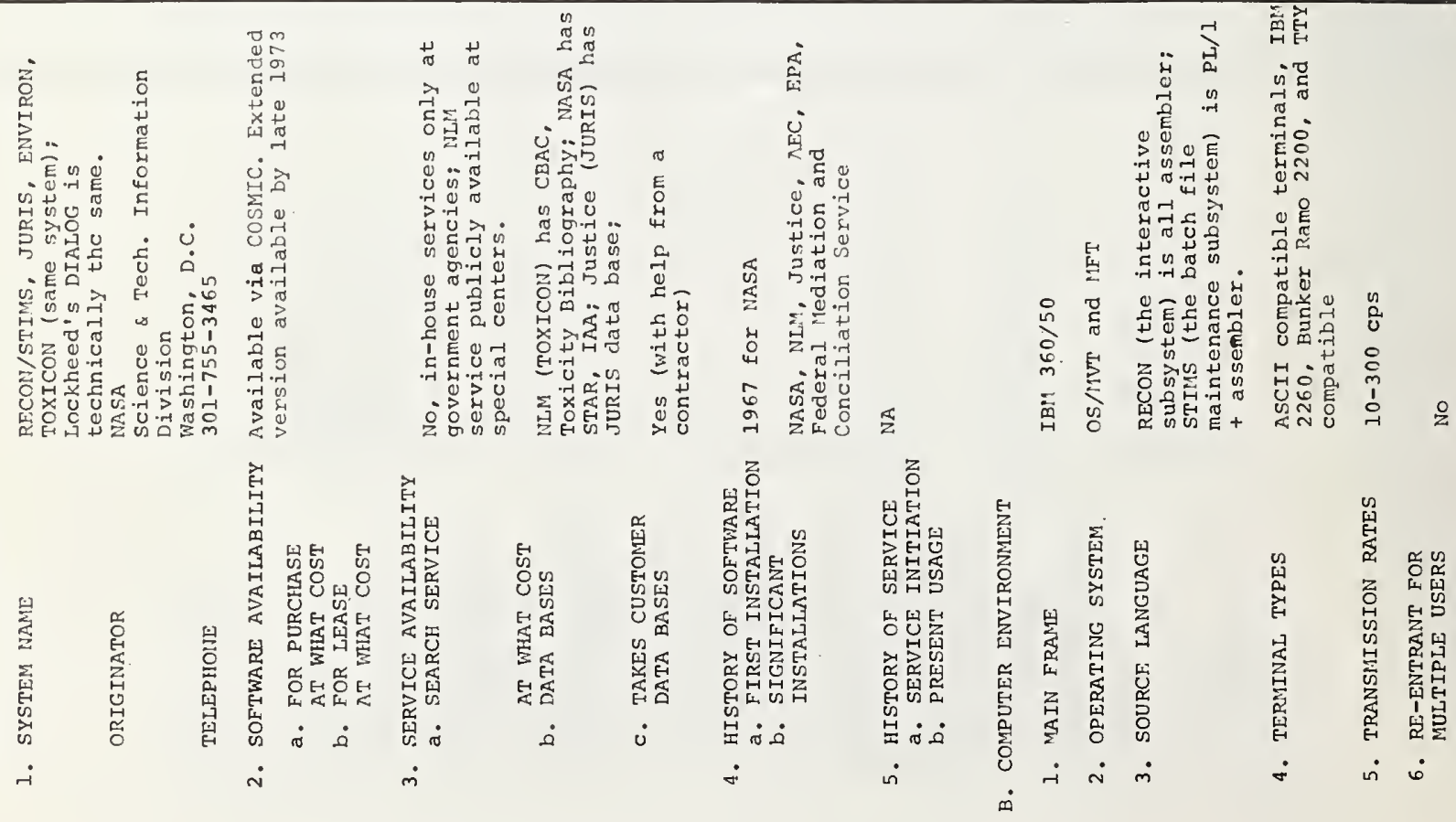




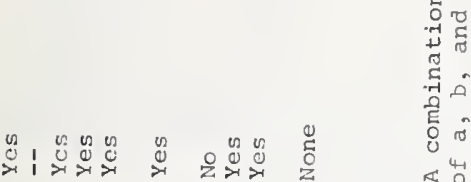

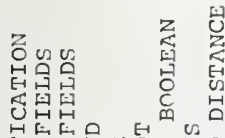

童

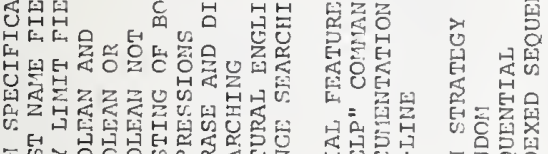

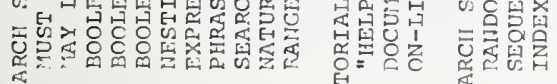

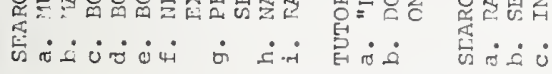

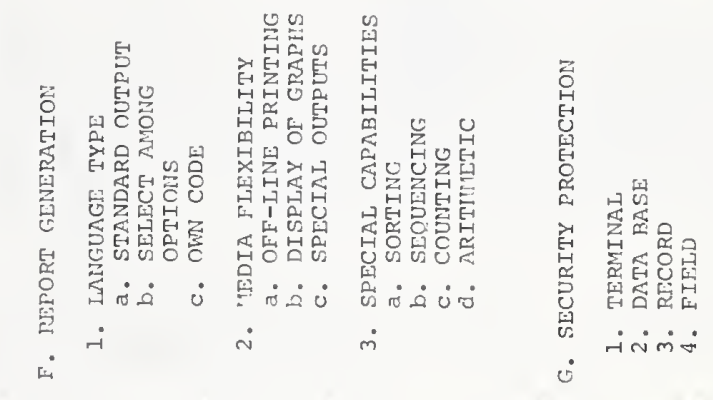

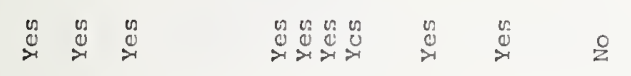

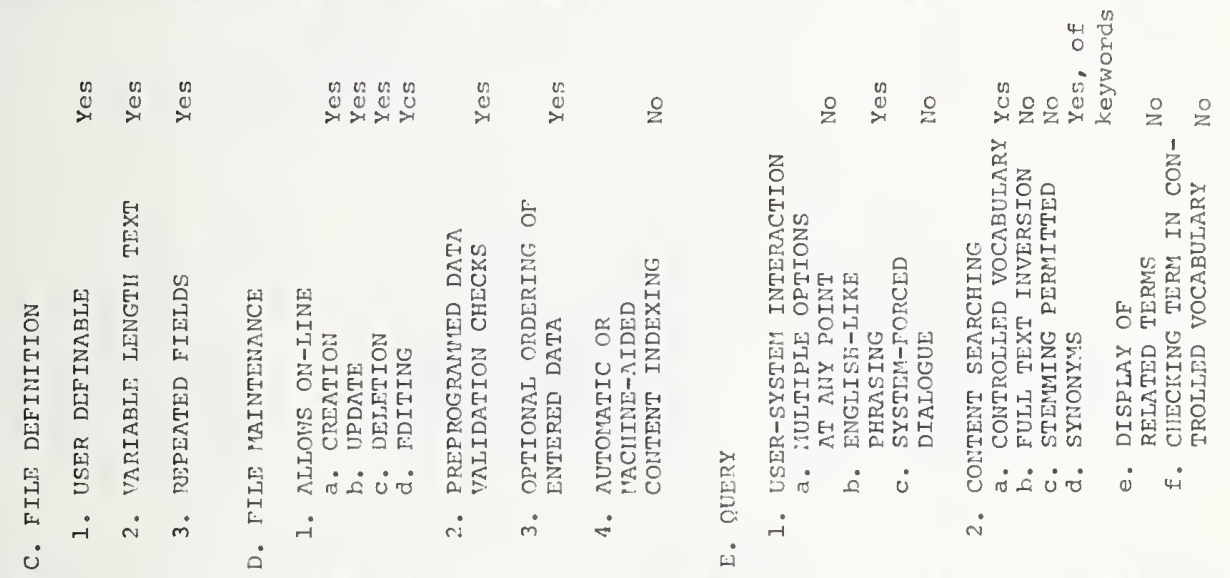

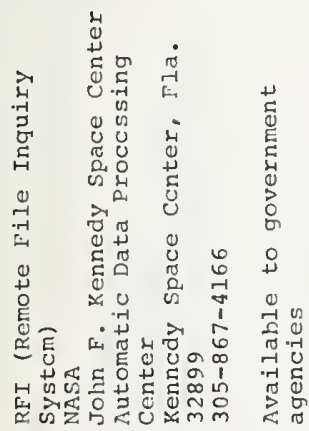

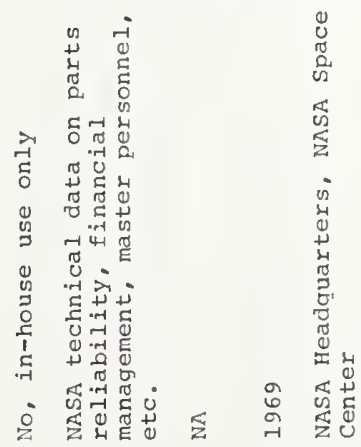

4

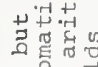

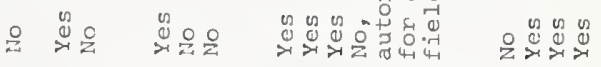
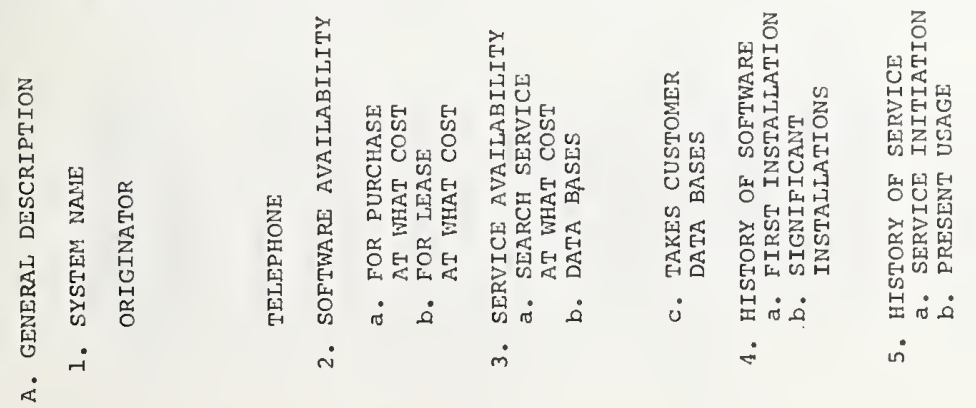

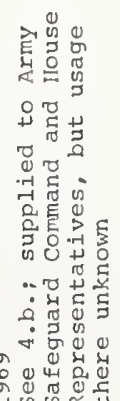

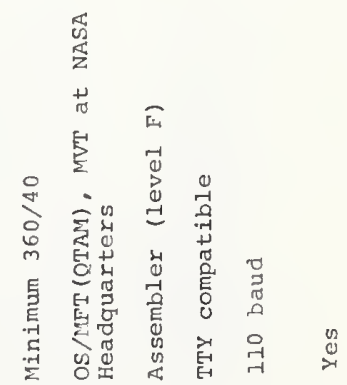

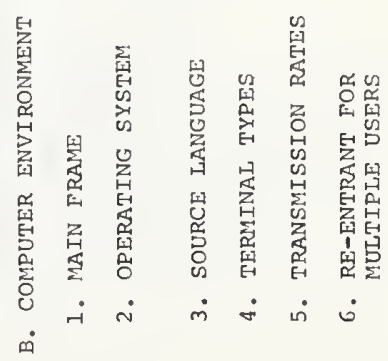




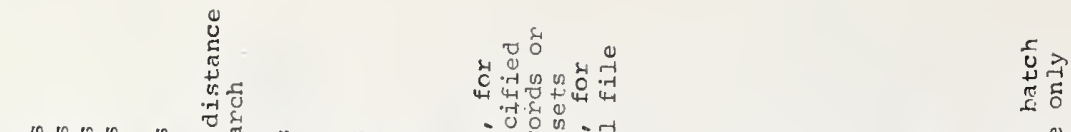

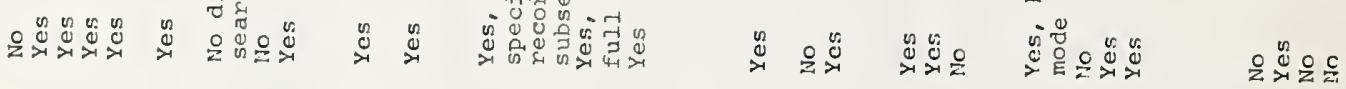

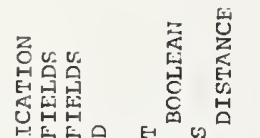

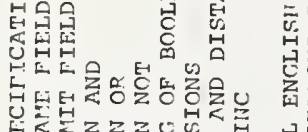

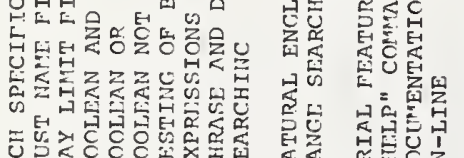

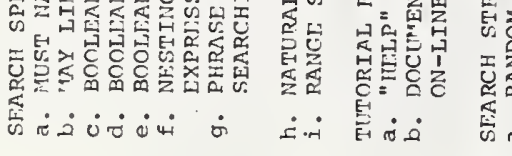

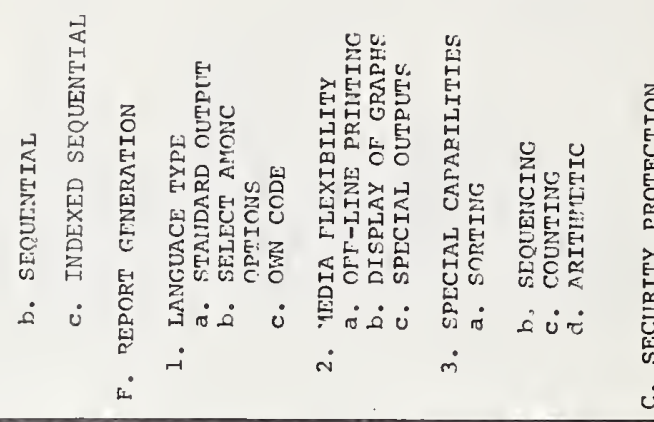

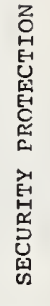

紫

的

点嵒曷

तinis:

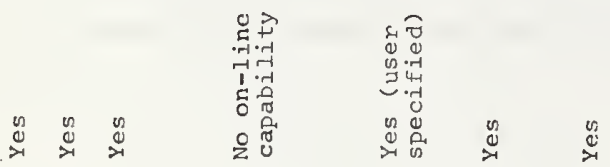

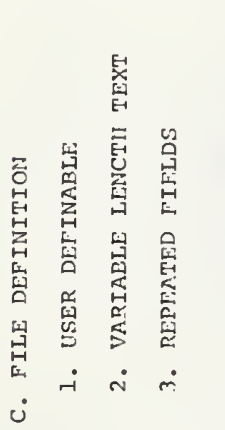

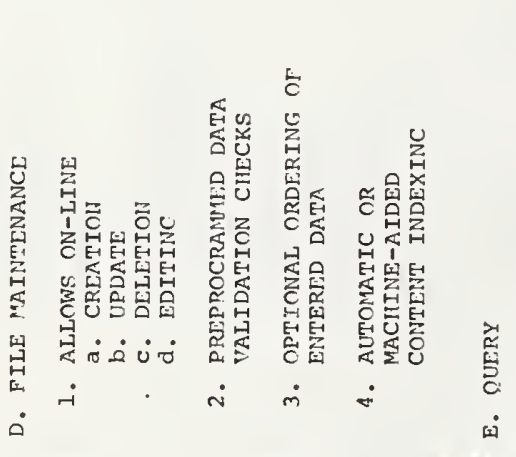

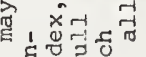

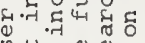

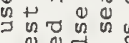

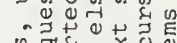

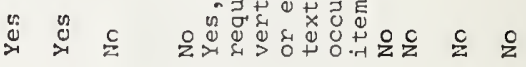

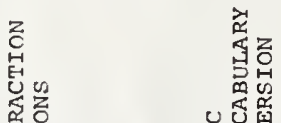

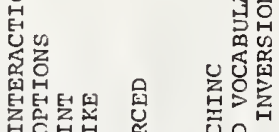

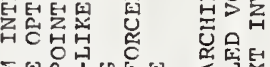

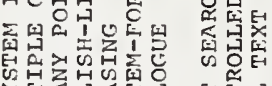

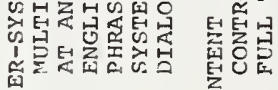

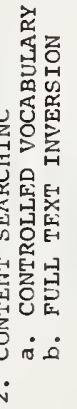

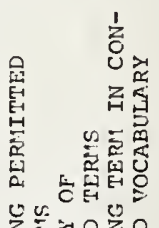

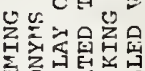
童础出出

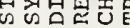
نं 0 क्ष

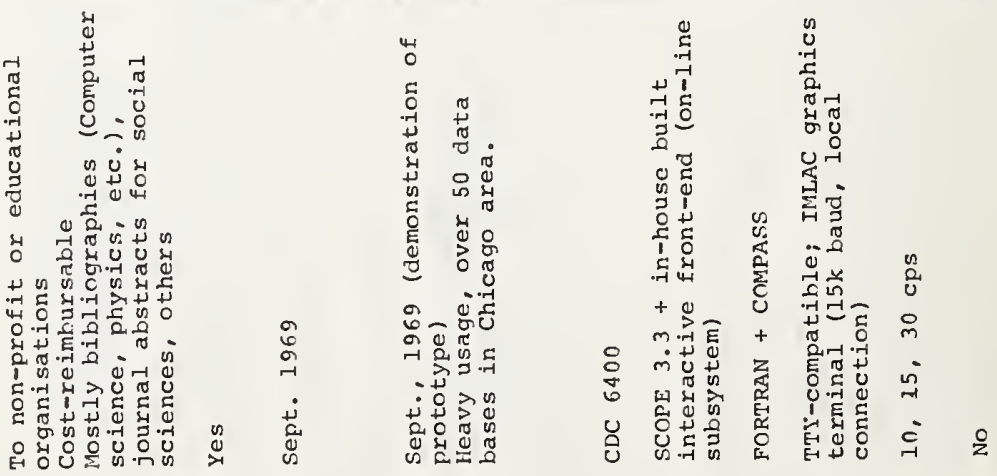

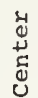

ก.

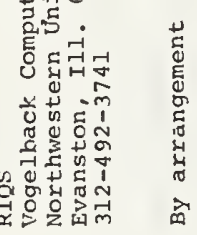

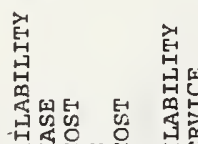

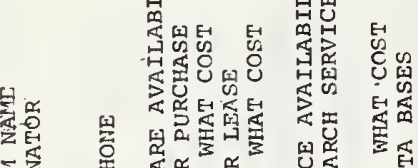

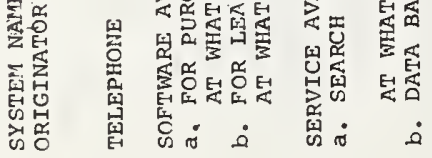

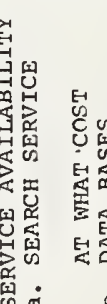

象星

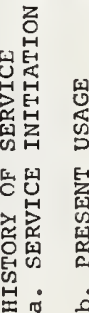

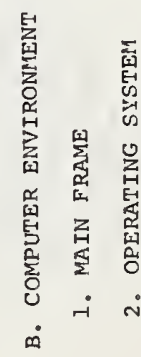

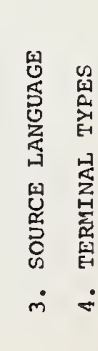

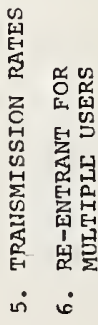




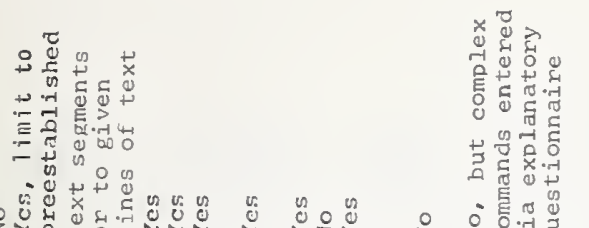

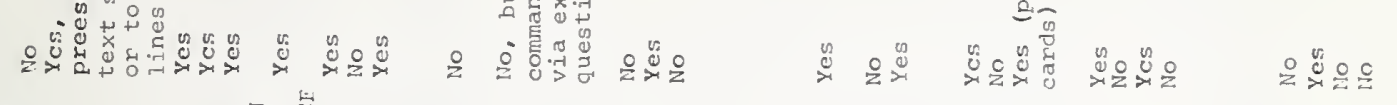

㩆能

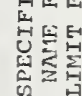

芯望

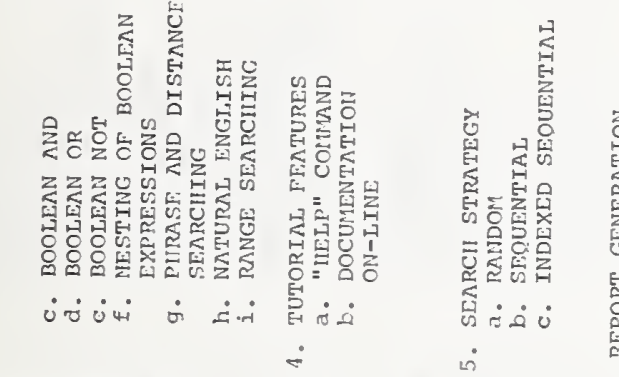

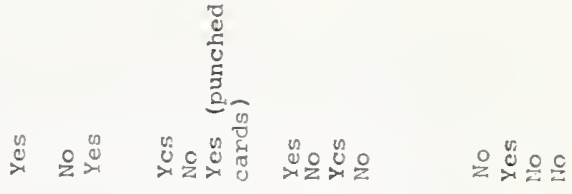

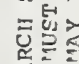

鼠这

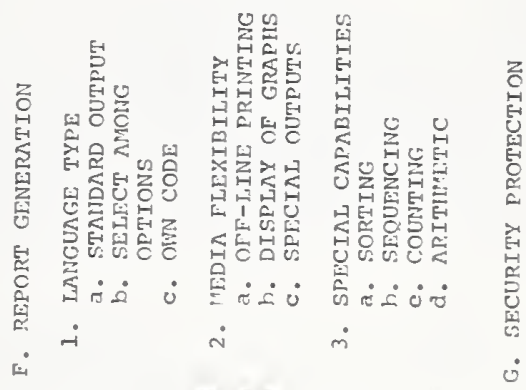

근

空是曷

$\dot{m}$

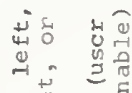

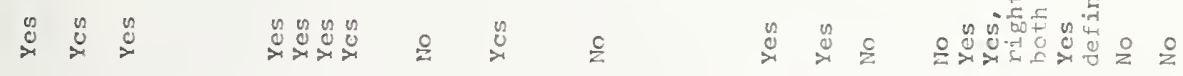
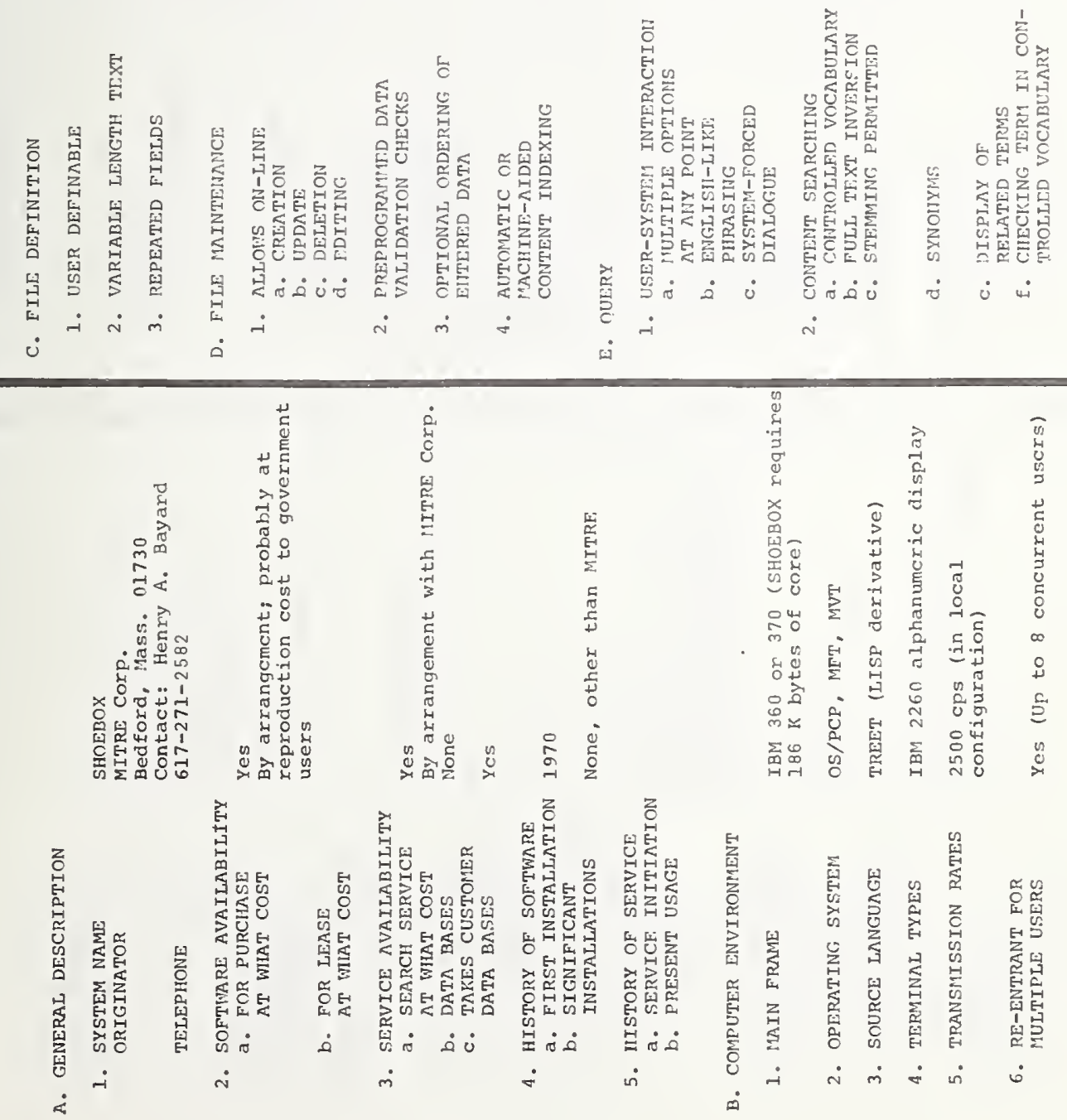


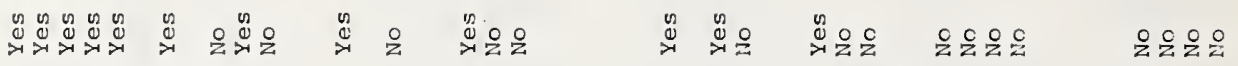
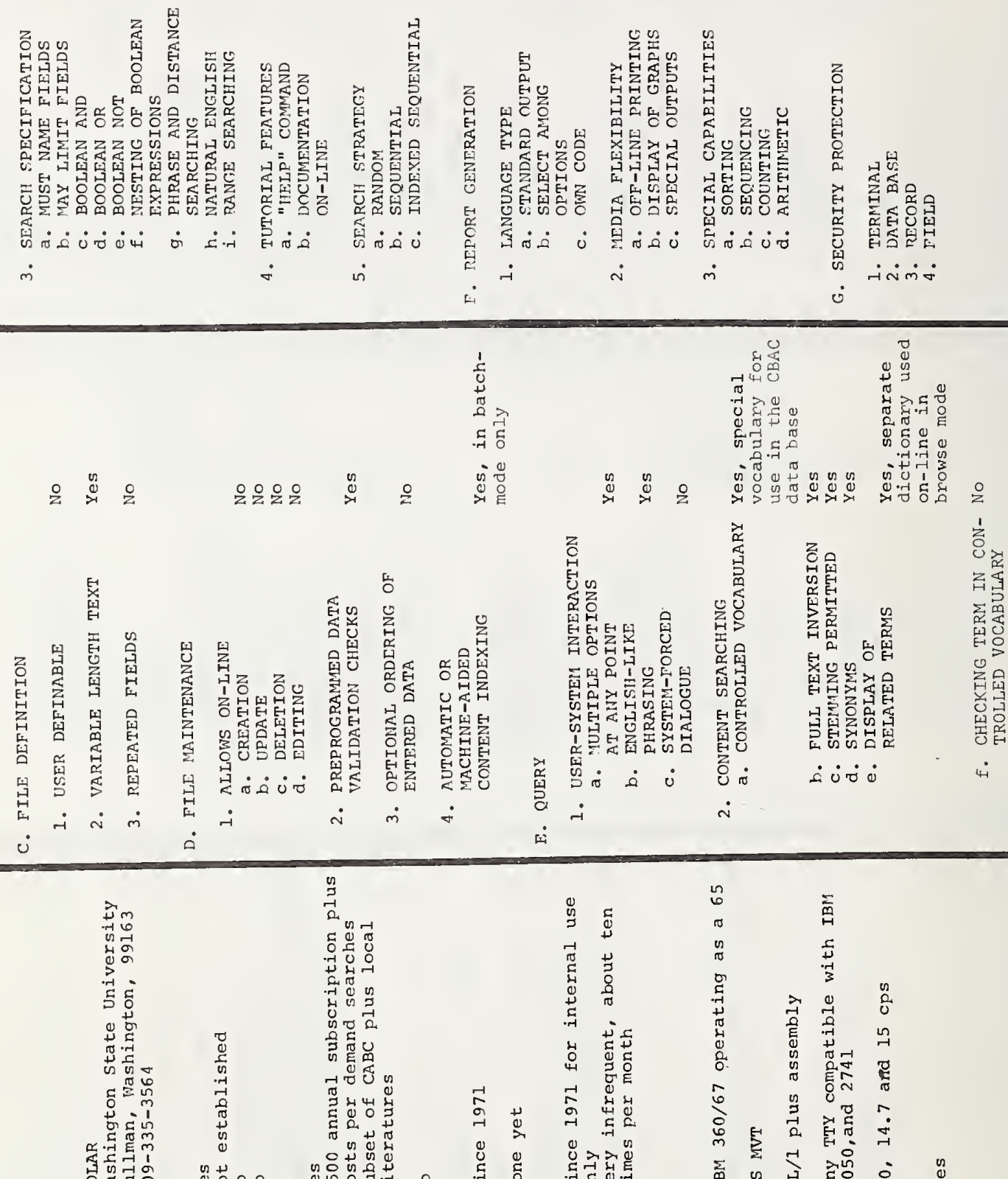

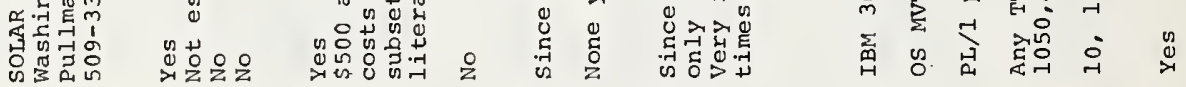

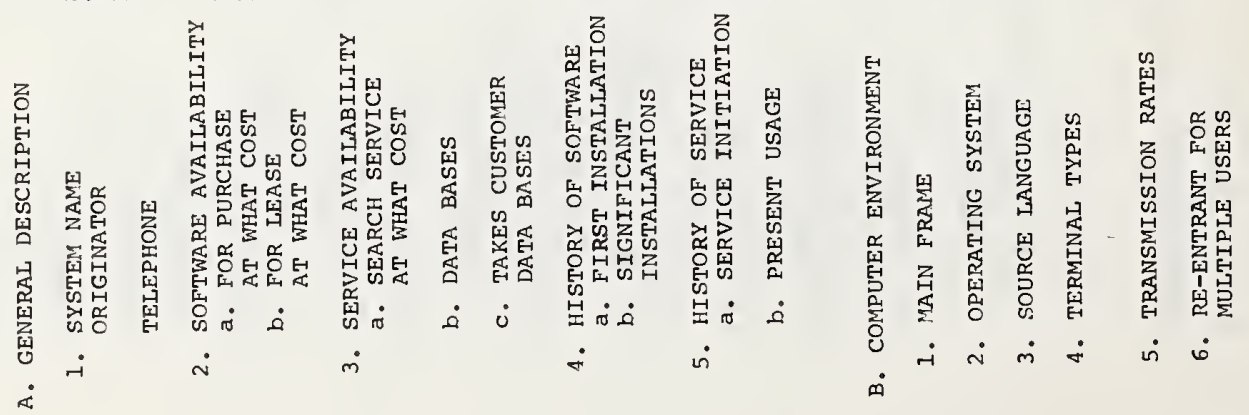




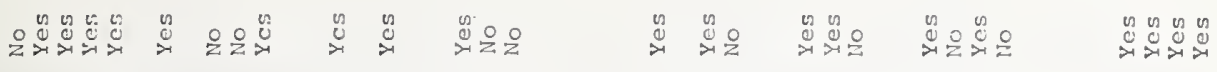

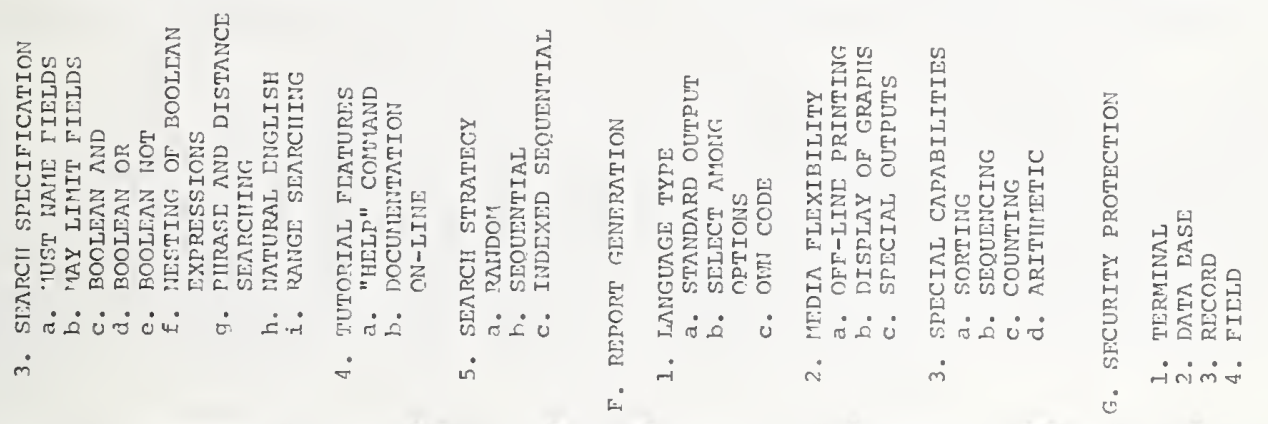

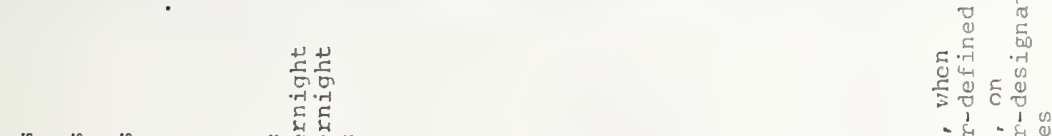

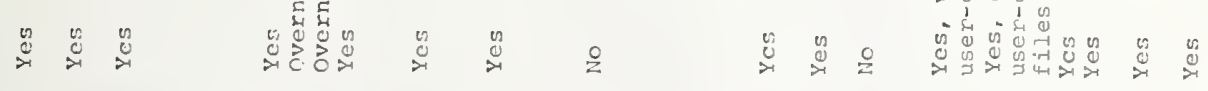
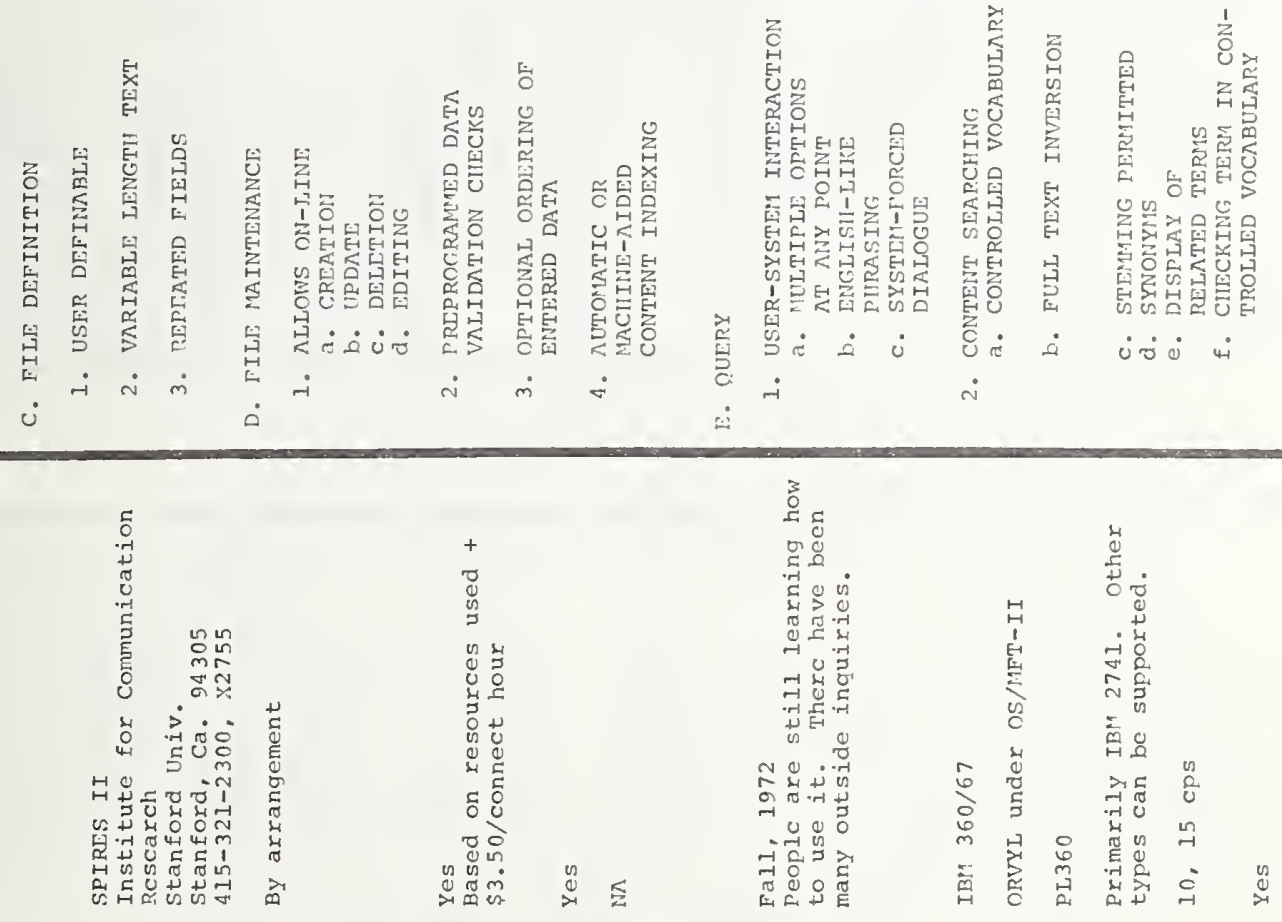

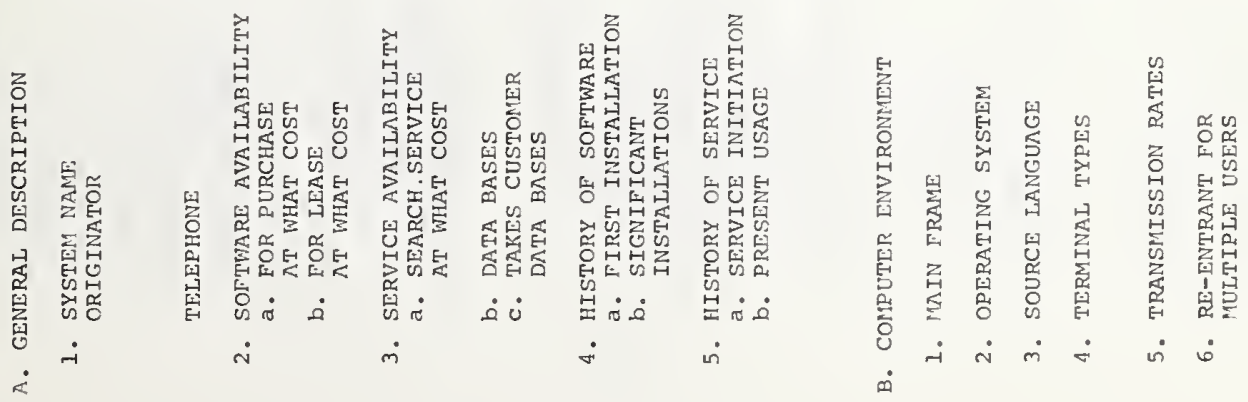




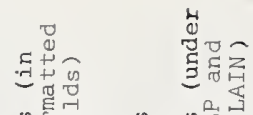

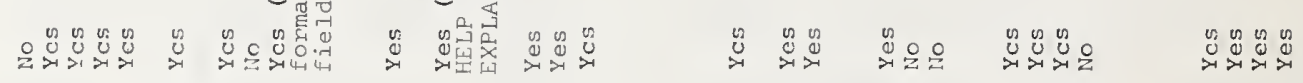
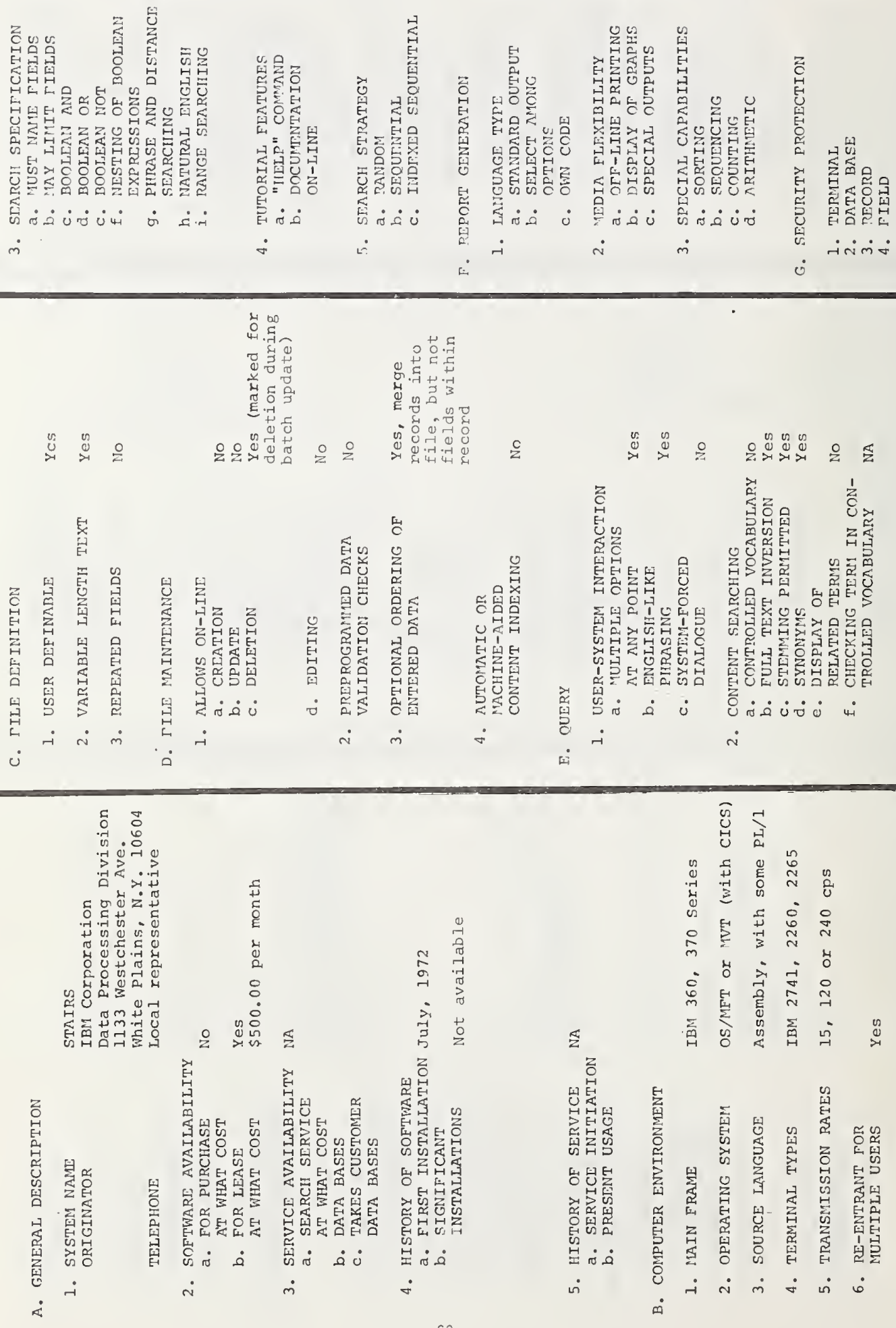


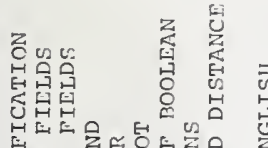

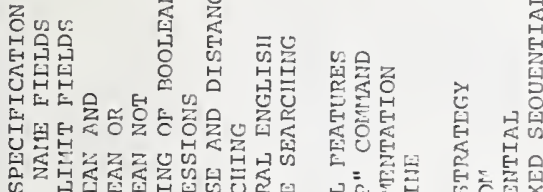
2:

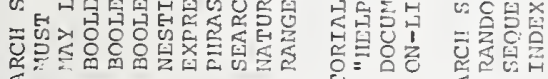

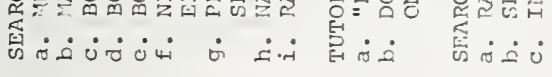
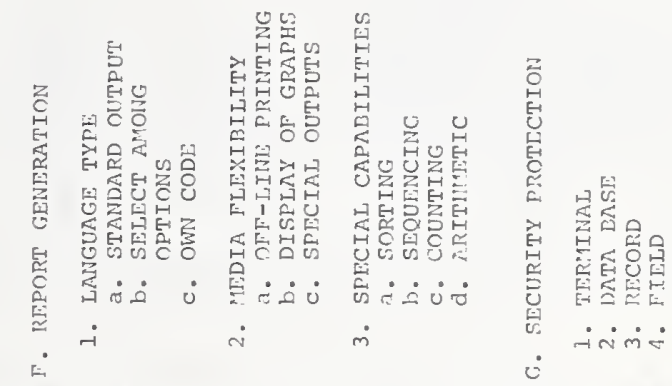

:

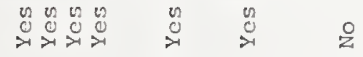

있

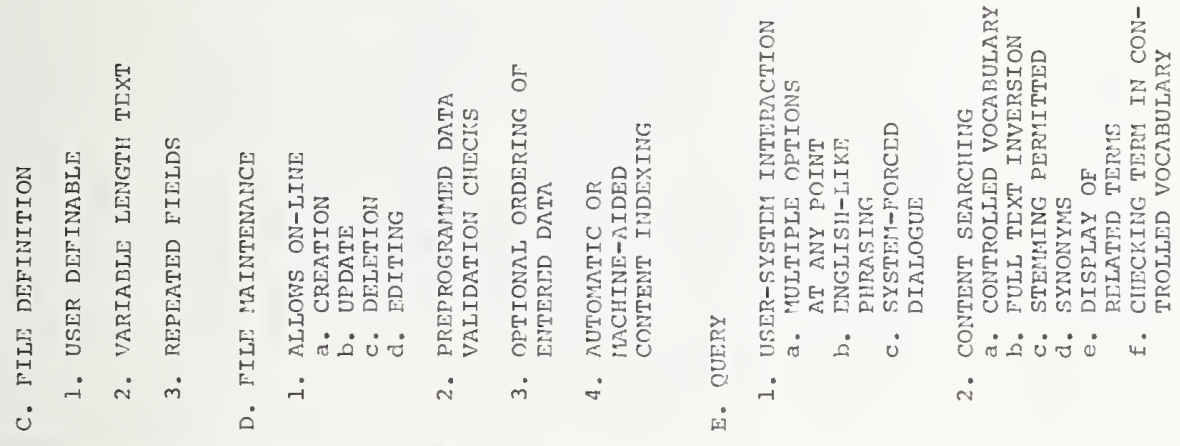

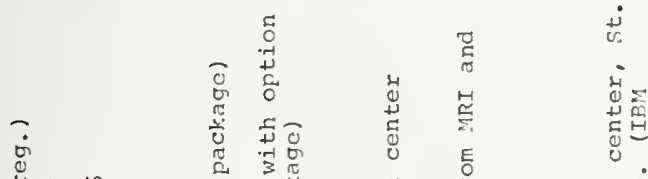

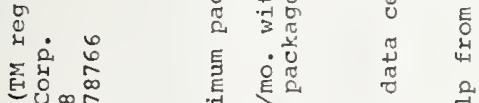

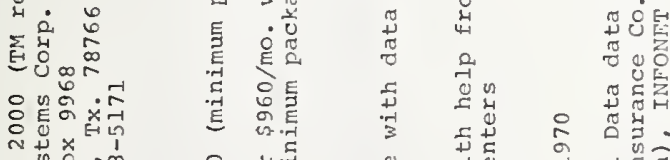

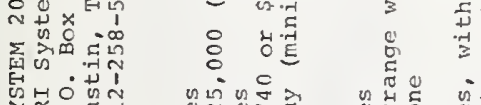

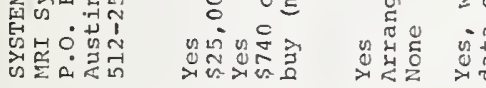

望

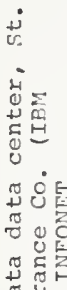

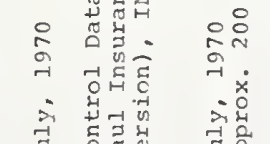

-

就

९ु

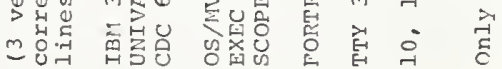

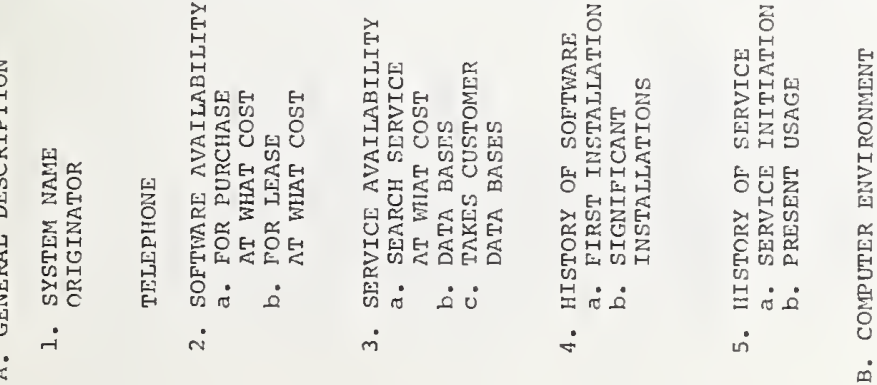




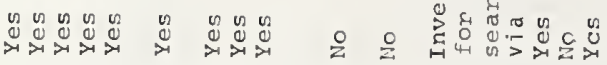
离

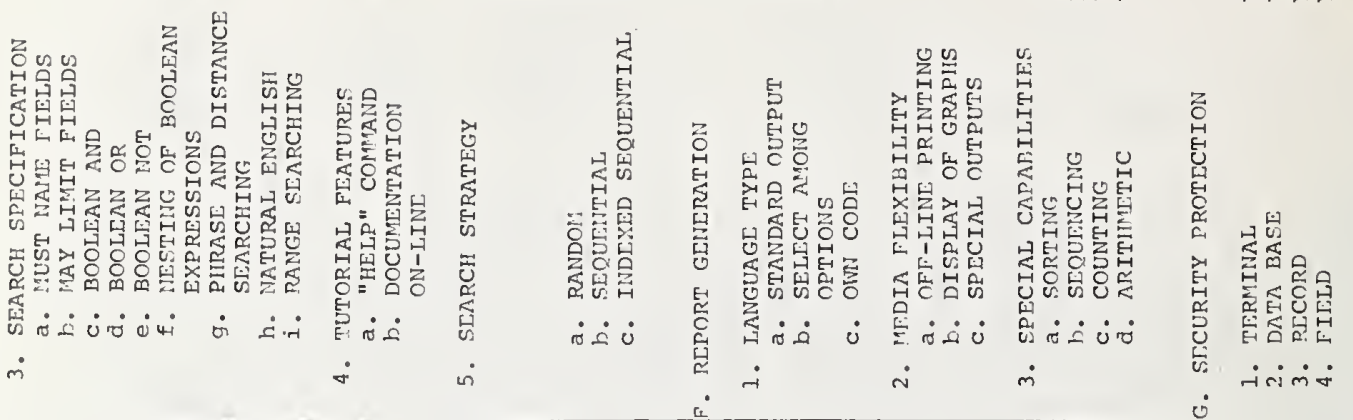

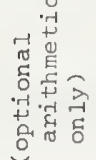

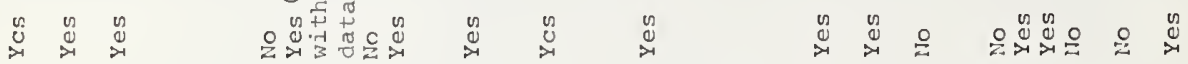
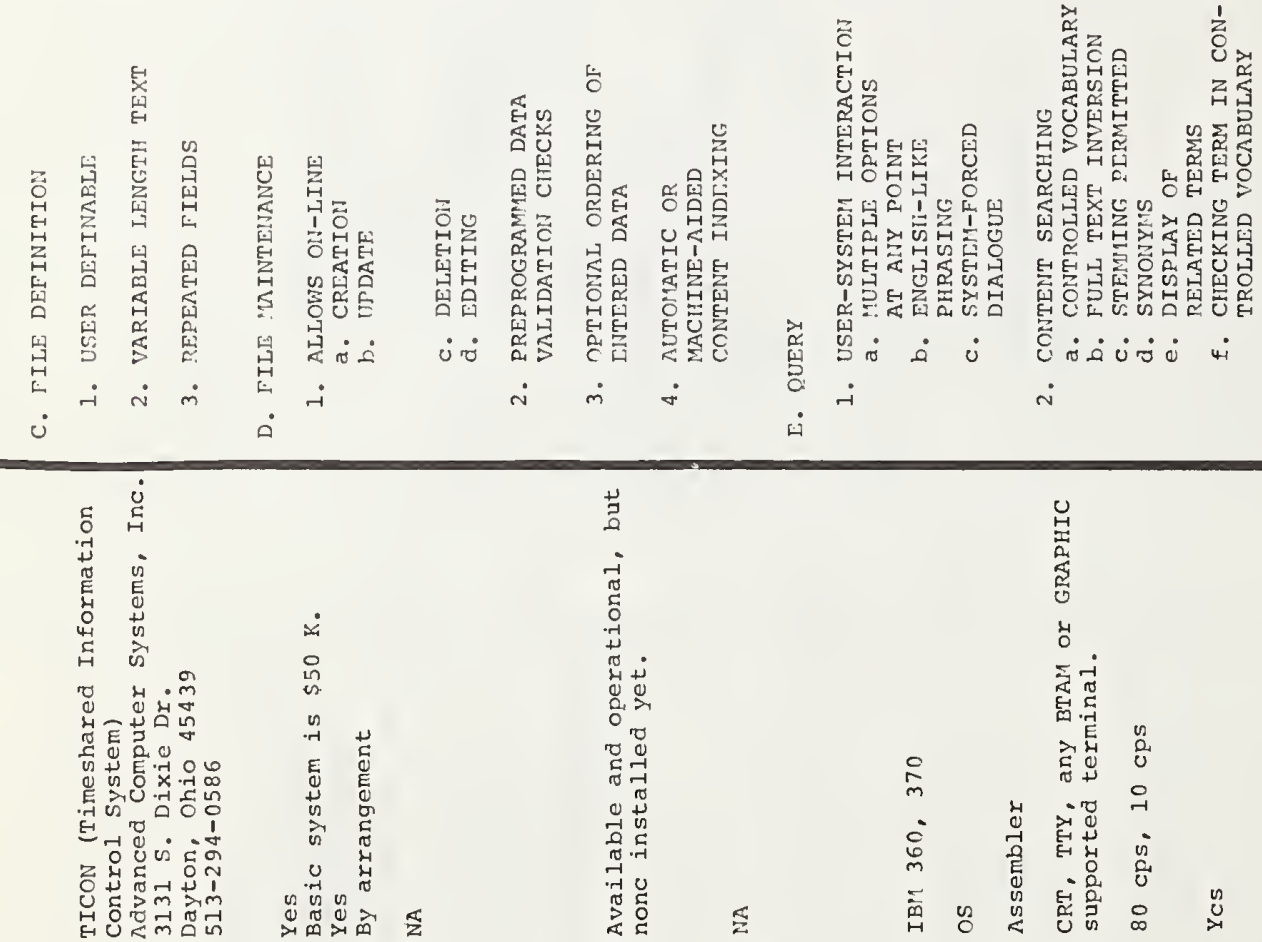

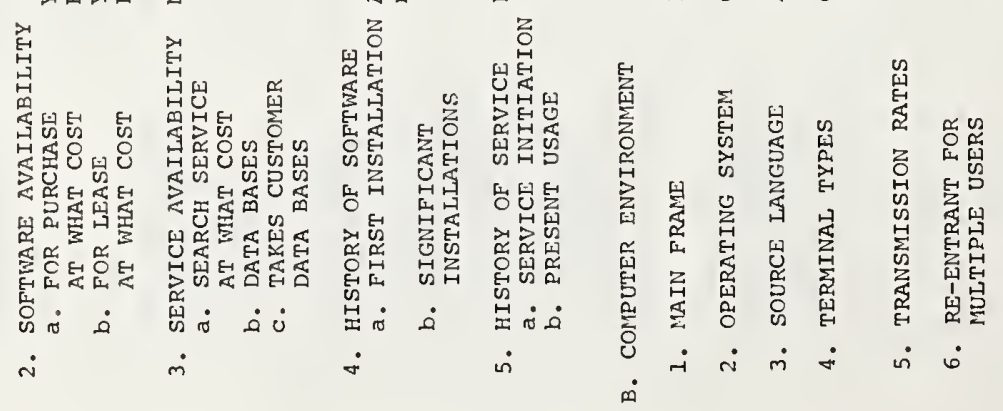




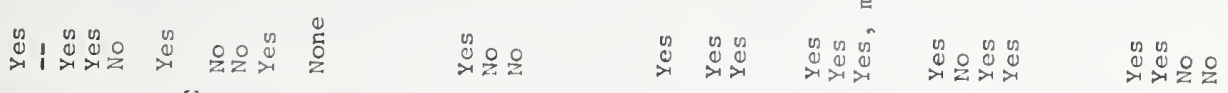

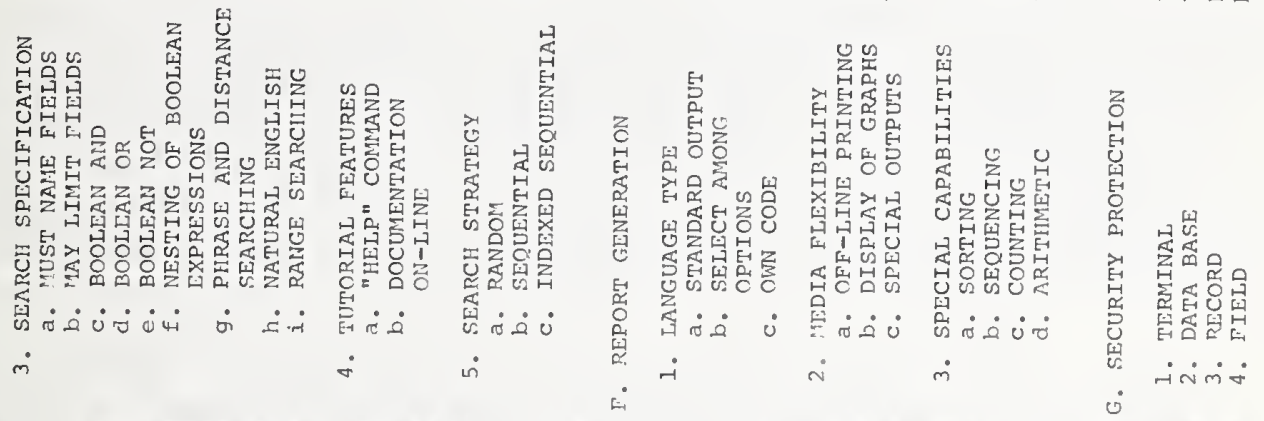
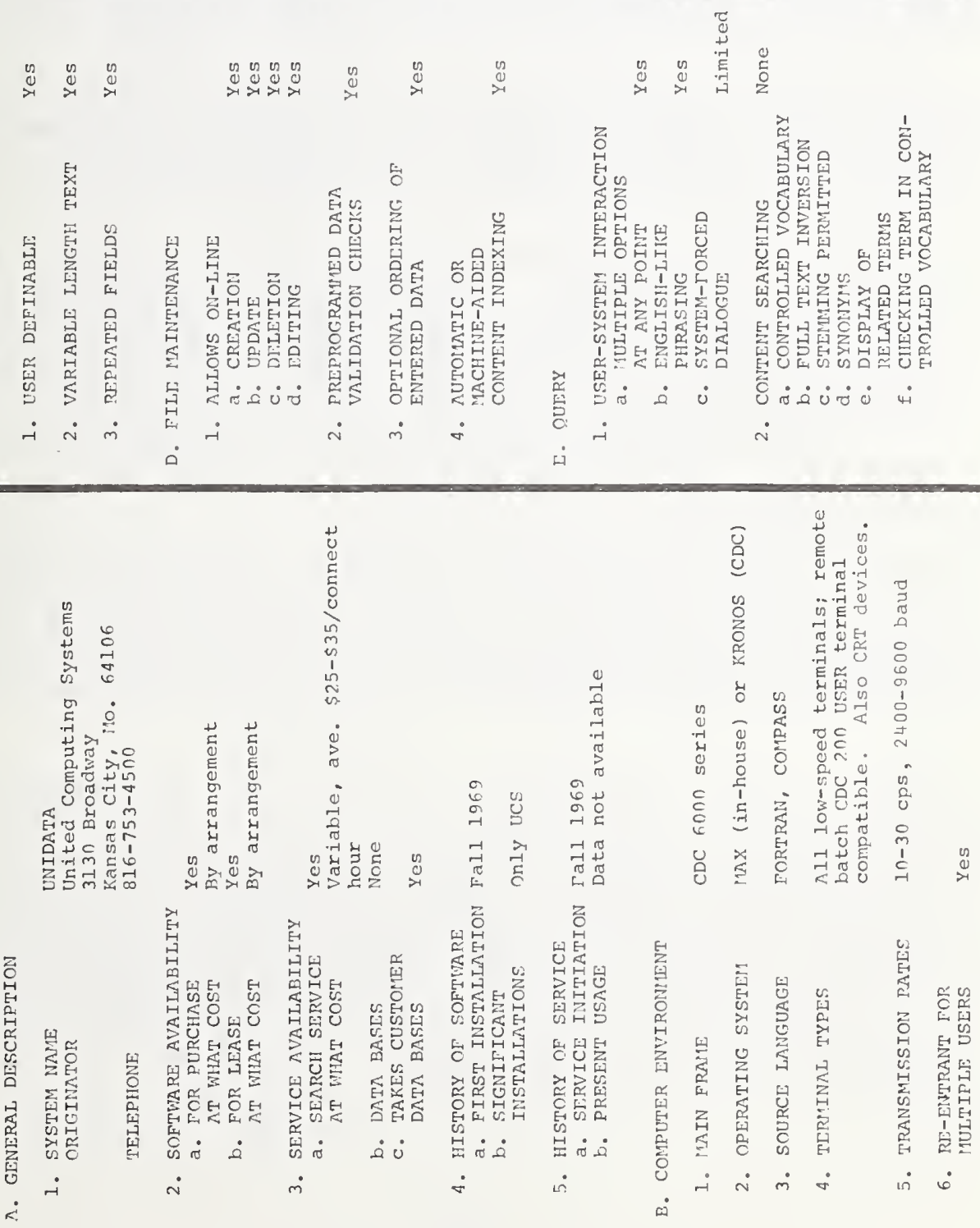


\section{GLOSSARY OF COMMON DATA BASES}

APA - American Psychological Association has created a data base containing the past four years' Psychological Abstracts.

COMPENDEX - Computerized Engineering Index, issued monthly by Engineering Index, Inc., is the computer-readable version of the printed publication, Engineening Index Monthly, which contains references spanning all engineering disciplines. These references are taken from professional and trade journals, publications of engineering organizations, papers from conferences and symposiums, books, and other documents.

CA-C - Chemical Abstracts Condensate issued by Chemical Abstracts Service is the computer searchable complement to the printed publication, Chemical Abstracts (CA), which covers the full range of chemistry, referencing 250,000 articles per year.

CA-Condensates is issued weekly; the content corresponds to an issue of CA. The tape version, CA-Condensates, precedes the cormesponding printed issue of $\mathrm{CA}$ by several weeks due to the time required to print, bind, and distribute CA printed issues.

The abstracts in $\mathrm{CA}$ and $\mathrm{CA}-\mathrm{Condensates}$ are grouped into five categories: Biochemistry, Organic Chemistry, Macromolecular Chemistry, Applied Chemistry and Chemical Engineering, and Physical and Analytical Chemistry. The first two groupings are published as an odd numbered issue one week, and the last three groupings are published as an even numbered issue the following week. Searches may be limited to odd or even numbered issues if desired.

CAIN - A data base distributed by the National Agricultural Library, it covers literature from all over the world concerned with agriculture in a broad sense. Ten per cent of the coverage consists of monographs; the remaining $90 \%$ consists of journal articles. The $90 \%$ comes from a review of 1,200 journals.

CT - Chemical Titles, also issued by Chemical Abstracts Service, contains journal references to approximately 4,500 articles per issue appearing in 650 important U. S. and non-U. S. chemical and engineering journals. Titles that appear in CT represent over $65 \%$ of the total abstracts that later appear in Chemical Abstracts. Chemical Titles offers journal references to articles approximately 70 days before their abstracts are published in Chemical Abstracts. In many cases titles appear in Chemical Titles before the journal containing the article is published. Thus Chemical Titles is valuable as an alerting service. 
CBAC - Chemical-Biological Activities, also issued by Chemical Abstracts Services, provides up-to-date coverage of published work concemed with the interaction of organic compounds (drugs, pesticides, etc.) with biological systerns.

Also covered are metabrlism studies and studies of in-vitro chemical reactions of biochemical interest. Nearly 700 journals are monitored. CBAC is a consolidated sounce of information in the chemical-biological field, and especially appeals to scientists involved in biochemical and physiological research.

The tape version of CBAC comesponds to the Chemical-Biological Activities hard-copy version; however, tape issues are usually available two-four weeks ahead of the printed copy. Each issue contains 500-600 digests.

ENVIRON - Environmental Information Retrieval On-line is a modified version of RECON operating at Environmental Protection Agency $(E P A)$. It services the EPA and maintains a data base of 8 separate files as foliows:

(I) Selected literature reference on noise abatement and control.

(2) Selected literature on industrial waste.

(3) Data and abstracts on on-going EPA contracts and grants researich effort.

(4) Data and abstracts on final reports of EPA contracts and grants research effort.

(5) Information on oil spills.

(6) Information on lists of potentially toxic chemicals.

(7) Information on water quality monitoring stations and parameters.

(8) Information on administrative records.

EHIC - Educational Resources Information Center is a nation-wide information network for acquiring, selecting, abstracting, indexing, storing, retrieving, and disseminating the most significant and timely educational research reports and projects. It is a project of the U. S. Office of Education, and produces two data bases, RIE (Research in Education) and CIJE (Curment Index to Journals in Education).

IAA - See STAR

INSPEC - A data base distributed by England's Institution of Electrical Engineers, it covers the fields of physics, electrical and electronic engineering, computers, and control engineering. Seventy-five percent of the coverage comes from a review of about 2,000 joumals. The other $25 \%$ are reports, monographs, patents, etc.

MARC II - is a data base containing bibliog:aphic citations prepared by the Library of Congress. 
NTIS - is a data base issued by National Technical Information Service of the U. S. Department of Commerce. It consists of unclassified govermment reports resulting from government-sponsored research. The reports are compiled in a publication now called Government Reports Announcements (GRA). GRA is available in machine readable form from the Department of Commerce.

The tapes contain standard bibliographic information such as author and title, as well as abstracts, descriptor terms, and information on how to order copies. The information consists mainly of science, engineering and mathematics, but also includes reports on behavioral and social sciences.

JURIS - Justice Retrieval and Inquiry System is a data base developed by the U. S. Department of Justice. It is designed to serve lawyers for the Federal govermment in their research process. The data base contains selected legal periodicals and reported cases, advisory opinions, supreme court briefs, solicitor general's memoranda, selected appellate briefs, selected legal memoranda, legal correspondence, legal memoranda on points of authority, agency general counsel specialized briefs and memoranda, etc.

MEDLINE - MEDLARS On-line data base is a special subset of the MEDLARS file, developed by the National Library of Medicine through their screening and analysis of the world's biomedical literature. MEDLINE covers over 1,100 biomedical journals from 1970 to present, whereas MEDLARS covers 2,400 biomedical journals from 1961 to present. The current file contains 400,000 records, and approximately 12,000 new records are added monthly.

PANDEX - The PANDEX tape contains curment index to scientific and technical literatures appearing in more than 2400 journals. The bibliographic information includes the title of the articles, name (s) of the author(s), name of the journal, Coden abbreviation, issue number, page number, and subject heading taken from a PANDEX thesaurus. PANDEX is produced by CCM Information Corporation, New York.

SPIN (Searchable Physics Information Notices) - a data base distributed monthly by the American Institute of Physics, it covers over onehalf of the world's physics and astronomy literature. Over 30,000 items are included every year from a journal coverage of 70 journals.

STAR - Scientific And Technical Aerospace Reports and International Aerospace Abstracts - are two data bases issued by NASA. They contain reports and journal articles that the NASA scientific and technical information program has collected. These data bases represent significant findings of Government, industrial, and academic researchers throughout the world on matters of interest to the aerospace community. 
TOXICON - A data base collected by the Toxicology Information Program, National Library of Medicine of the Department of Health, Education and Welfare. It contains bibliographic citations of literature primarily in the area of toxicology, pharmacology and environmental health. The citations include all the principal components of a literature reference; namely, author, title, journal, joumal coden, volume, issue, pagination and year. Most references are annotated with index terms, an abstract or both. Where possible and appropriate, Chemical Abstracts Service Registry Numbers are made part of the annotation. 


\section{BIBLIOGRAPHY}

There have been a number of surveys and other works done in recent years which are similar in intent to ours but not identical in scope or form. A selective bibliography is now presented.

[1] Anderson, R. K., "Index of Data Management Software Packages," National Bureau of Standards, Report 10 932, Oct. 1972.

[2] Auerbach Corporation, "Methodology for Evaluating Data Management Systems," Auerbach Report 5081-TR-1, undated.

[3] CODASYL Systens Comnittee, "A Survey of Generalized Data Base Management Systems," CODASYL Technical Report, May 1969.

[4] CODASYL Systems Committee, "Feature Analysis of Generalized Data Base Management Systems," CODASYL Technical Report, May 1971.

[5] Fong, E., "A Survey of Selected Document Processing Systems," National Bureau of Standards Technical Note 599, Oct. 1971.

[6] Fry, J. P., et. al., "Data Management Systems Survey," MITRE Corporation Report MTP-329, Jan. 1969.

[7] Koehr, G. J., et. al., "Data Management Systems Catalog," MITRE Corporation Report MTP-139, Jan. 1973.

[8] Marron, B., et. al., "A Study of Six University-Based Information Systems," Technical Note 781, National Bureau of Standards, June 1973.

[9] Seiden, H. R., "A Comparative Analysis of Interactive Storage and Retrieval Systerns with Implications for BCN Design," System Development Corporation Technical Memorandum 4421, Jan. 1970.

[10] Walker, D. E. (editor), Interactive Bibliographic Search: The User/Computer Interface, AFIPS Press, 1971. 
FORM NBS-114A (1-71)

\begin{tabular}{|c|c|c|}
\hline $\begin{array}{l}\text { 1. PUBLICATION OR REPORT NO. } \\
\text { NBS TN }-819\end{array}$ & $\begin{array}{l}\text { 2. Gov't Accession } \\
\text { No. }\end{array}$ & 3. Recipient's Accession No. \\
\hline \multirow{2}{*}{\multicolumn{2}{|c|}{$\begin{array}{l}\text { 4. TIT LE AND SUBTITLE } \\
\text { A TECHNICAL INDEX OF INTERACTIVE INFORMATION SYSTEMS }\end{array}$}} & $\begin{array}{l}\text { 5. Publication Date } \\
\text { March } 1974\end{array}$ \\
\hline & & 6. Performing Organization Code \\
\hline $\begin{array}{l}\text { 7. Author(s) Dennis W. Fife, Kirk Rankin, Elizabe } \\
\text { Justin C. Walker, Beatrice A. Marmon }\end{array}$ & Fong, & 8. Performing Organization \\
\hline \multicolumn{2}{|l|}{ 9. PERFORMING ORGANIZATION NAME AND ADDRESS } & $\begin{array}{l}\text { 10. Project/Task/Work Unit No. } \\
64 \mathrm{C}\end{array}$ \\
\hline \multicolumn{2}{|l|}{$\begin{array}{l}\text { NATIONAL BUREAU OF STAND ARDS } \\
\text { DEPARTMENT OF COMMERCE } \\
\text { WASHINGTON, D.C. } 20234\end{array}$} & 11. Cuntract/Grant No. \\
\hline \multirow{2}{*}{\multicolumn{2}{|c|}{$\begin{array}{l}\text { 12. Sponsoring Organization Name and Address } \\
\qquad \begin{array}{l}\text { National Science Foundation } \\
1800 \text { G Street, NW } \\
\text { Washington, D. C. } 20550\end{array}\end{array}$}} & $\begin{array}{l}\text { 13. Type of Report \& Period } \\
\text { Covered } \\
\text { Final }\end{array}$ \\
\hline & & 14. Sponsoring Agency Code \\
\hline
\end{tabular}

15. SUPPLEMENTARY NOTES

16. ABSTRACT (A 200-word or less factual summary of most significant information. If document includes a significant bibliography or literature survey, mention it here.)

This report constitutes a reference to technical features and operational status of interactive information systems, i.e. those providing a 'conversational' usage mode to a 'non-programmer' through a data terminal device. It is aimed at the ADP service manager, for his use in the state-of-the-art assessments preparatory to a detailed system selection process. It contains an index that describes 46 systems in terms of a list of over 50 technical features plus descriptive, identification, and background information. In addition, there are aids and examples contributing to the intended use of the index.

17. KEY WORDS (AIphabetical order, separated by semicolons) Bibliographic systems; computer programs; computer systems; data base; data management; information retrieval; information services; interactive system; query language; software selection; text processing.

\begin{tabular}{|c|c|c|}
\hline $\begin{array}{l}\text { 18. AVAILABILITY STATEMENT } \\
\mathrm{X} \text { UNLIMIT ED. }\end{array}$ & $\begin{array}{l}\text { 19. SECURITY CLASS } \\
\text { (THIS REPORT) } \\
\text { UNCL ASSIFIED }\end{array}$ & $\begin{array}{c}\text { 21. NO. OF PAGES } \\
73\end{array}$ \\
\hline $\begin{array}{l}\square \text { FOR OFFICIAL DIST RIBUTION. DO NOT RELEASE } \\
\text { TO NTIS. }\end{array}$ & $\begin{array}{l}\text { 20. SECUR ITY CLASS } \\
\text { (THIS PAGE) } \\
\text { UNCL ASSIFIED }\end{array}$ & $\begin{array}{l}\text { 22. Price } \\
\$ 1.20\end{array}$ \\
\hline
\end{tabular}




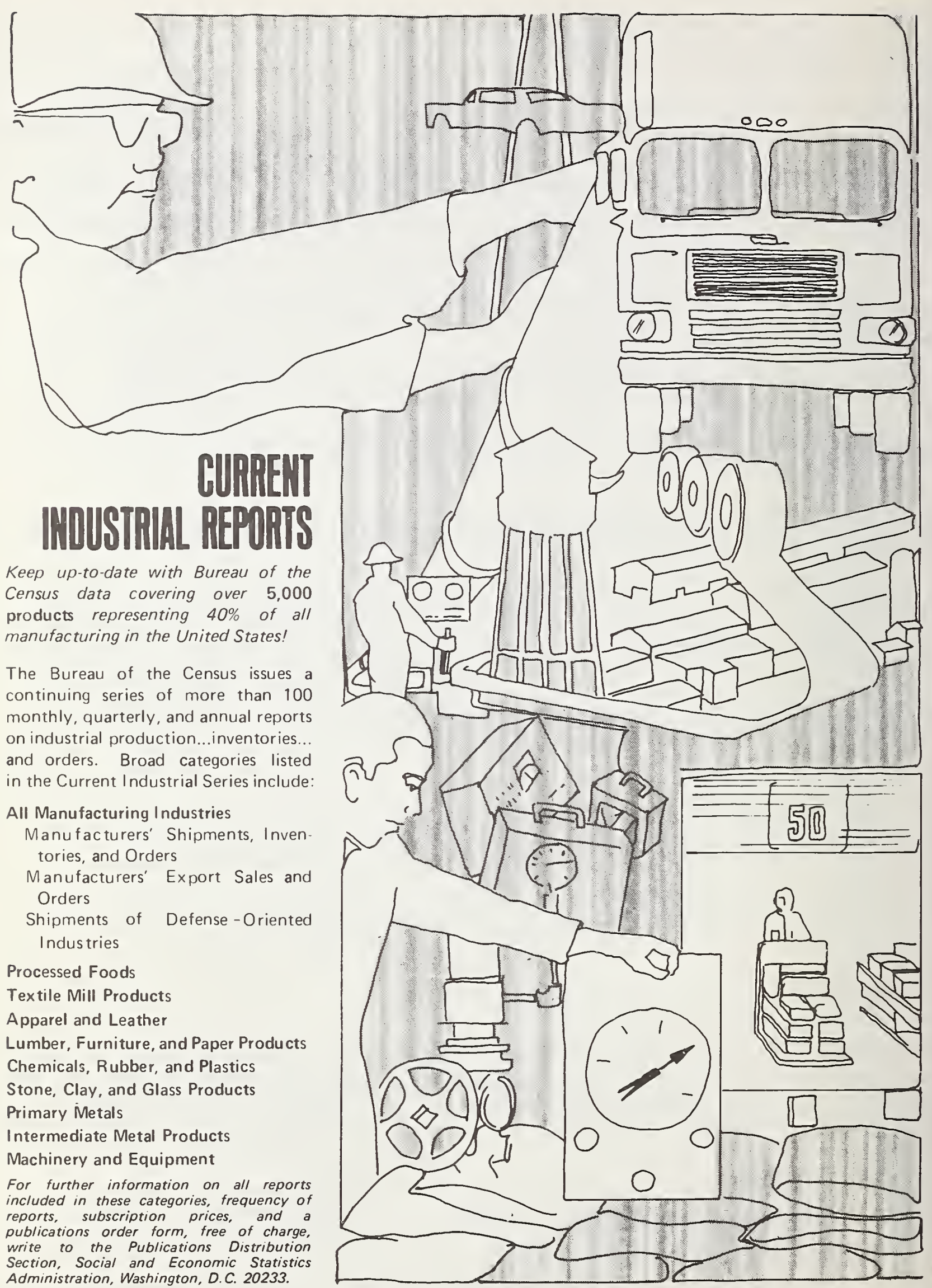



UPP-TO-DAIE
BUSINESS REPORTS

Cuppent Penof's on Pêlail Tpade

*Weekly Retail Sales-Estimates of weekly retail sales for the United States for selected major kind-of-business groups, including figures for the comparable weeks in the previous year. Issued each Thursday.

- Monthly Retail Trade Report-Estimates of monthly retail sales for the United States by major kind-ofbusiness groups and selected individual kinds of business; separate figures shown, in more limited kind-of-business detail, for firms operating 11 or more retail stores. Sum. mary sales data presented for geographic regions and divisions, and for 15 large States, the 20 large standard metropolitan statistical areas and the five largest cities. Also included are national estimates of endof-month accounts receivable balances for retail stores.

-Annual Retail Trade ReportEstimates of annual sales and purchases, and of yearend accounts receivable, balances and inventories held by retailers in the United States by major kind-of-business groups and selected individual kinds of business. Separate figures shown in more limited kindof-business detail for firms operating 11 or more retail stores. Also shown are salesinventory ratios and per capita sales by kind-of-business for the United States, by major kind-of-business groups. Per capita sales estimates are also shown in limited kind-of. business detail for geographic divisions, and for the larger States and standard metropolitan statistical areas.

*Advance Monthly Retail Sales-Advance estimates of monthly retail sales for the United States lincluding data adjusted for seasonal variations and trading day differences) are compiled by major kind-ofbusiness groups about 10 days after the month covered.

Annual Subscription $\$ 30.10$

tMonthly Department Store Sales for Selected AreasMonthly dollar sales volume and the percent change in sales compared with the previous month and the same month in the previous year; cumulative year-to-date comparisons with data for the previous year. The number of department stores in the current month is also shown. Data are collected in about 200 standard metropolitan statistical areas, cities, and other areas.

Annual Subscription $\$ 1.00$

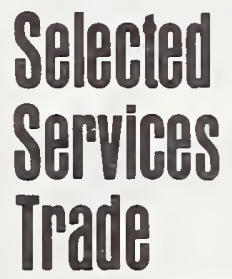

tMonthly Selected Services Receipts-This report provides data on monthly receipts of six major kind-of-business groups of services: Hotels, motels, tourist courts, trailer parks, and camps; personal services; business services; auta motive services; miscellaneous repair services; and motion picture, amusement, and recreation services. Comparable data for the previous months and for the same month in the previous year are also shown, in addition to the percent changes. Data are shown both unadjusted and adjusted for seasonal variations and trading day differences.

Annual Subscription $\$ 1.00$
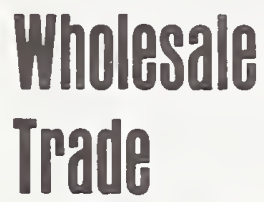

* Monthly Wholesale Trade Report-This report includes estimated dollar sales, end-ofmonth inventories, and stocksales ratios of merchant wholesalers, by kind of business for the current month, with comparisons for previous months. Dollar volume sales estimates are shown by geographic division in total and for durable and nondurable kind-ofbusiness subtotals. Sales and inventory trends (percent changes) are shown by detailed kinds-of-business at the national level and for selected kinds-of-businesses by geographic division. Measures of sampling variability are given. United States data are shown adjusted for seasonal variations and in the case of sales, also for trading day differences.

Annual Subscription $\$ 5.15$
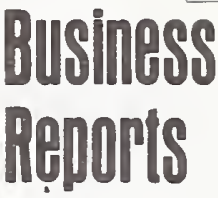

tCanned Food Report-This report is issued for five datesJanuary 1, April 1, June 1, July 1, and November 1 - to show total stocks of wholesale distributors and canners, including warehouses of retail multiunit organizations, of selected canned food items. In the January 1 report, sepa rate data are shown for the No. 10 can size as well as for warehouse stocks of retail multiu nit organizations.

Annual Subscription $\$ 1.00$

tGreen Cofiee Inventories and Roastings-This quarterly report provides estimates of green coffee inventories held by roasters, importers, and dealers, the quantity of green coffee roasted, and the amount roasted for soluble use, by quarters, for the current and previous 3 years. Also included are quarterly imports of green coffee.

\section{Annual Subscription $\$ 1.00$}

*Available from the Superintendent of Documents, U.S. Government Printing Office, Washington, D.C. 20402.

†Available from the Publications Distribution Section, Social and Economic Statistics Administration, Washington, D.C. 20233

Publications Order Forms furnishing additional information of the various reports listed here are available free of charge from the Publications Distribution Section, Social and Economic Statistics Administration, Washington, D.C. 20233. 


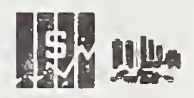

P.23 SPECIAL STUDIES.

Infrequent reports on methods, concepts, or specialized data.

In addition to the findings of the Census of Population, conducted every 10 years, the Bureau of the Census publishes continuing and up-todate statistics on population counts, characteristics, and other special studies on the American people. Data are issued in the eight separate series of reports described herein and released under the general title, Current Population Reports.

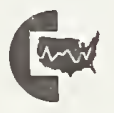

\section{P.20 POPULATION}

CHARACTERISTICS.

Current national and, in some cases, regional data on geographic residence and mobility, fertility, education, school enrollment, marital status, numbers and characteristics of household and families, etc. Approximately 10 reports each year.

\section{P.25 POPULATION \\ ESTIMATES AND \\ PROJECTIONS.}

Monthly estimates of the total population of the United States; annual mid-year estimates of the population of States, by broad age groups, and of the United States by age, color, and sex; annual estimates of the components of population change. Estimates of the population of selected metropolitan areas and their component counties. Projections of the future population of the United States and individual States. Approximately 20 reports each year.

\section{P-26 FEDERAL-STATE} COOPERATIVE PRO. GRAM FOR POPULATION ESTIMATES.

Population estimates for counties for selected States in which the figures are prepared by a State agency as part of the Federal-State Cooperative Program for Local Population Estimates.

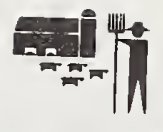

P.27 FARM POPULATION.

(Census-ERS). Data on the size and selected characteristics of the farm population of the United States. Issued jointly with the Economic Research Service, U.S. Department of Agriculture. One or two reports each year.

P-28 SPECIAL CENSUSES.

Results of population censuses taken at the request and expense of city or other local governments. Subscription includes only the quarterly and annual summaries showing the total population figures for all the censuses conducted during the particular period. Individual reports issued for areas of 50,000 or more, showing the population by age, sex, and color are available separately. See "How to Order Reports." Five reports each year.

\section{P-60 CONSUMER} INCOME.

Information on the proportions of families and persons at various income levels. Also data on the relationship of income to age, sex, color, family size, education, occupation, work experience, and other characteristics. Two reports each year.

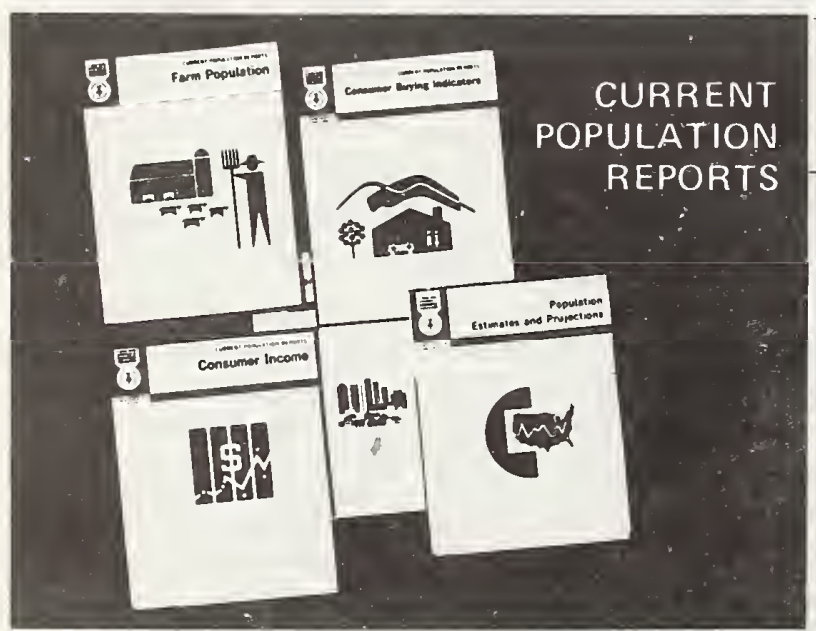

P.65 CONSUMER

BUYING INDICATORS.

Information on household ownership and recent purchase of cars, trucks, and selected household equipment. Ownership and purchase data are shown by income, age of head, residence, race, and other household characteristics. Annually.

\section{HOW TO ORDER} REPORTS.

All Current Population Reports are sold as a single subscription for $\$ 30.50$ a year ladd $\$ 7.75$ for foreign mailing). Price includes Series P-20, P-23, P-25, P-26, P-27, P-28 (quarterly and annual summaries only), P-60, and P-65.

Subscriptions to individual series are not sold separately, but single copies of reports issued in all series except P-28 may be obtained from Superintendent of Documents, U.S. Government Printing Office, Washington, D.C. 20402. Individual city or area reports in the P-28 series may be ordered from the Social and Economic Statistics Administrationprices vary. For information on CP reports, write to Publications Distribution Section, Social and Economic Statistics Administration, Washington, D.C. 20233.

Mail order form with payment to:

Superintendent of Documents

U.S. Government Printing Office

Washington, D.C., 20402

or any U.S. Dept. of Commerce District Office

Please send__ subscription(s) of the Current Population Reports Series as described above, at $\$ 30.50$ per subscription. (8 Series). Add $\$ 7.75$ for foreign mailing.

C56.218 (Nos.)

Enclosed is $\$$ (check, money order, Supt. Docs. coupons) or charge Deposit Account No.

Name

Street Address

City, State, ZIP.

Make check or money order payable to Superintendent of Documents 




\section{NBS TECHNICAL PUBLICATIONS}

PERIODICALS

JOURNAL OF RESEARCH reports National Burcau of Standards research and development in physics, mathematics, and chemistry. Comprehensive scientific papers give complete details of the work, including laboratory data, experimental procedures, and theoretical and mathematical analyses. Illustrated with photographs, drawings, and charts. Includes listings of other NBS papers as issued.

Published in two sections, available separately:

\section{- Physics and Chemistry (Section A)}

Papers of interest primarily to scientists working in these fields. This section covers a broad range of physical and chemical research, with major emphasis on standards of physical measurement, fundamental constants, and properties of matter. Issued six times a year. Annual subscription: Domestic, $\$ 17.00$; Foreign, \$21.25.

\section{- Mathematical Sciences (Section B)}

Studies and compilations designed mainly for the mathematician and theoretical physicist. Topics in mathematical statistics, theory of experiment design, numerical analysis, theoretical physics and chemistry, logical design and programming of computers and computer systems. Short numerical tables. Issued quarterly. Annual subscription: Domestic, $\$ 9.00$; Foreign, $\$ 11.25$.

\section{DIMENSIONS, NBS}

The best single source of information concerning the Bureau's measurement, research, developmental, cooperative, and publication activities, this monthly publication is designed for the layman and also for the industry-oriented individual whose daily work involves intimate contact with science and technology - for engineers, chemists, physicists, research managers, product-development managers, and company executives. Annual subscription: Domestic, \$6.50; Foreign, $\$ 8.25$.

\section{NONPERIODICALS}

Applied Mathematics Series. Mathematical tables, manuals, and studies.

Building Science Series. Research results, test methods, and performance criteria of building matcrials, components, systems, and structures.

Handbooks. Recommended codes of engineering and industrial practice (including safety codes) developed in cooperation with interested industries, professional organizations, and regulatory bodies.

Special Publications. Proceedings of NBS conferences, bibliographies, annual reports, wall charts, pamphlets, etc.

Monographs. Major contributions to the technical literature on various subjects related to the Bureau's scientific and technical activities.

National Standard Reference Data Series. NSRDS provides quantitative data on the physical and chemical properties of materials, compiled from the world's literature and critically evaluated.

Product Standards. Provide requirements for sizes, types, quality, and methods for testing various industrial products. These standards are developed cooperatively with interested Government and industry groups and provide the basis for common understanding of product characteristics for both buyers and sellers. Their use is voluntary.

Technical Notes. This series consists of communications and rcports (covering both other-agency and NBS-sponsored work) of limited or transitory interest.

Federal Information Processing Standards Publications. This series is the official publication within the Federal Government for information on standards adopted and promulgated under the Public Law 89-306, and Bureau of the Budget Circular A-86 entitled, Standardization of Data Elements and Codes in Data Systems.

Consumer Information Series. Practical information, based on NBS research and experience, covering areas of interest to the consumer. Easily understandable language and illustrations provide useful background knowledge for shopping in today's technological marketplace.

\section{BIBLIOGRAPHIC SUBSCRIPTION SERVICES}

The following current-awareness and literature-survey bibliographies are issued periodically by the Bureau:

Cryogenic Data Center Current Awareness Service (Publications and Reports of Interest in Cryogenics). A literature survey issued weekly. Annual subscription: Domestic, \$20.00; foreign. \$25.00.

Liquefied Nätural Gas. A literature survey issued quarterly. Annual subscription: $\$ 20.00$.

Superconducting Devices and Materials. A literature survey issued quarterly. Annual subscription: $\$ 20.00$. Send subscription orders and remittances for the preceding bibliographic services to the U.S. Department of Commerce, National Technical Information Service, Springfield, Va. 22151.

Electromagnetic Metrology Current Awareness Service (Abstracts of Selected Articles on Measurement Techniques and Standards of Electromagnetic Quantities from D-C to Millimeter-Wave Frequencies). Issued monthly. Annual subscription: $\$ 100.00$ (Special rates for multi-subscriptions). Send subscription order and remittance to the Electromagnetic Metrology Information Center, Electromagnetics Division, National Bureau of Standards, Boulder, Colo. 80302.

Order NBS publications (exccpt Bibliographic Subscription Services) from: Supcrintendent of Documcnts, Government Printing Office, Washington, D.C. 20402. 
U.S. DEPARTMENT OF COMMERCE

National Bureau of Standards Washington, D.C. 20234

POSTAGE AND FEES PAID U.S. DEPARTMENT OF COMMERCE COM-2I5

Miss Yeates

NBS Library El20 Admin. Bldg.

Penalty for Private Use, $\mathbf{\$ 3 0 0}$

Miss Yeates
NBS Library El20 Admin. Bldg.

